\title{
Raising Boys' Achievement
}

Mike Younger and Molly Warrington

with

John Gray, Jean Rudduck, Ros McLellan, Eva Bearne, Ruth Kershner and Pat Bricheno

University of Cambridge Faculty of Education 


\title{
Research Report
}

No 636

\section{Raising Boys’ Achievement}

\author{
Mike Younger and Molly Warrington \\ with \\ John Gray, Jean Rudduck, Ros McLellan, \\ Eva Bearne, Ruth Kershner and Pat Bricheno \\ University of Cambridge Faculty of Education
}

The views expressed in this report are the authors' and do not necessarily reflect those of the Department for Education and Skills.

(c) Queen's Printer and Controller of HMSO 2005. Published with the permission of DfES on behalf of the Controller of Her Majesty's Stationery Office. Applications for reproduction should be made in writing to The Crown Copyright Unit, Her Majesty's Stationery Office, St Clements House, 2-16 Colegate, Norwich NR3 1BQ. 
Table of Contents

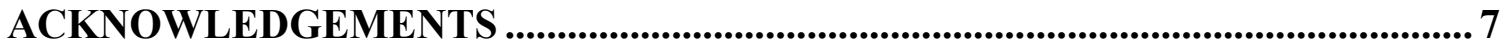

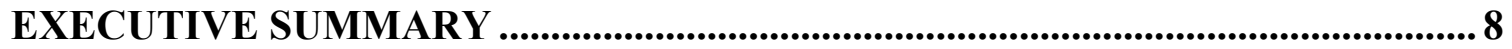

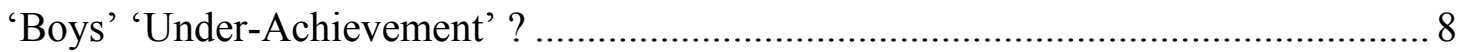

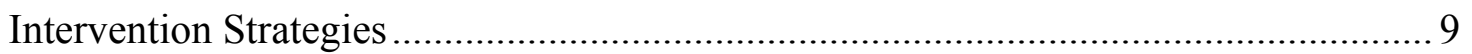

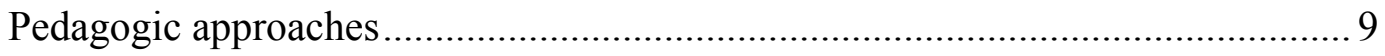

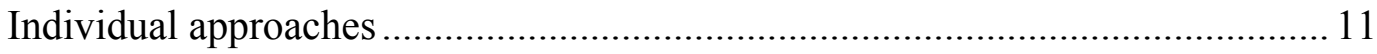

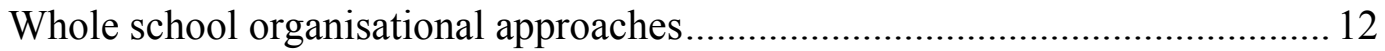

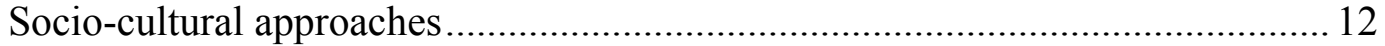

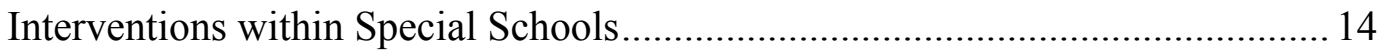

The Broader Issues .......................................................................................... 14

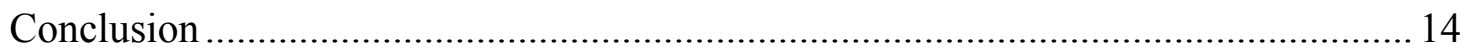

CHAPTER 1: The Context of the Project .................................................................. 16

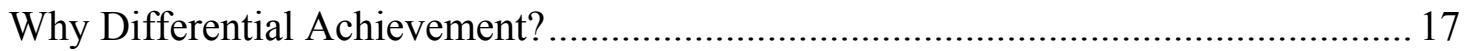

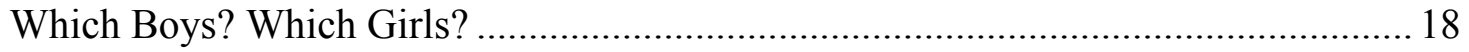

CHAPTER 2: The Gender Gap: Dilemmas and Debates ........................................ 20

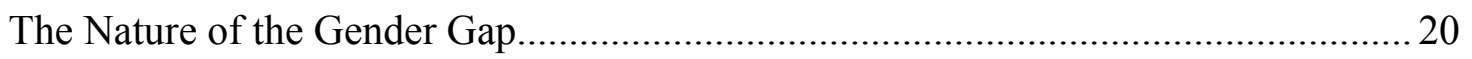

Interpreting the Gender Gap: the case for care and vigilance ................................ 25

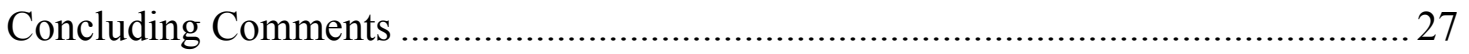

CHAPTER 3: Intervention Strategies ............................................................................. 28

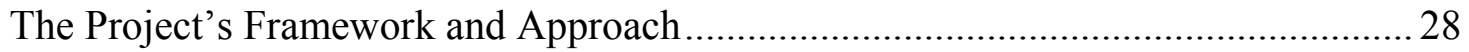

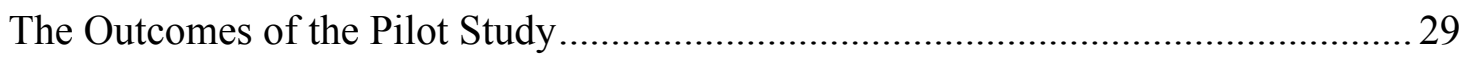

Research Design for the Intervention Stage ....................................................... 31

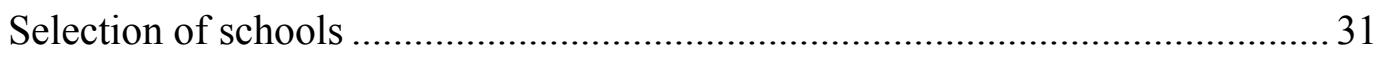

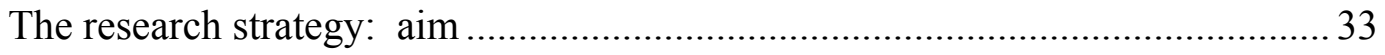

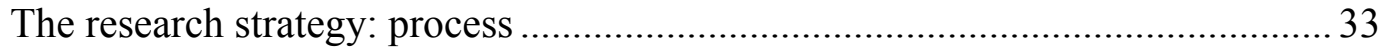

CHAPTER 4: Pedagogy I: Raising Achievement in Literacy at Key Stage 2......... 36

Approaches to Literacy: processes and products ................................................ 36

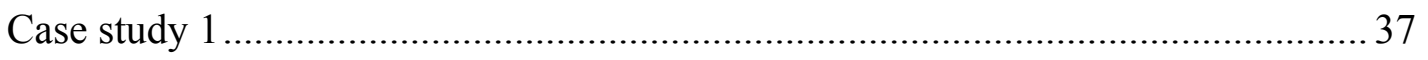

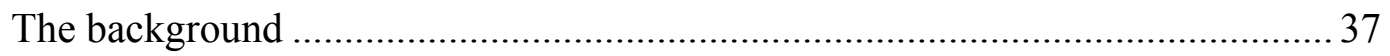

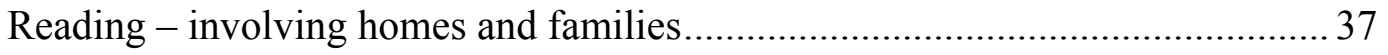

Assessing progress in reading at key stage 2 .................................................. 39 


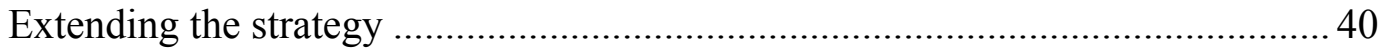

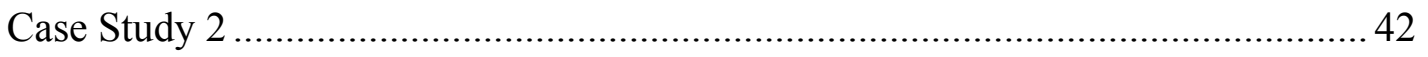

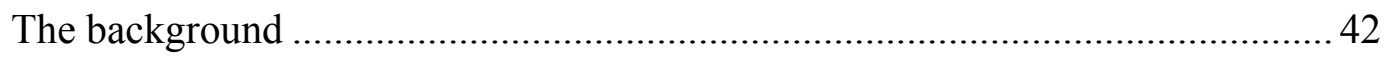

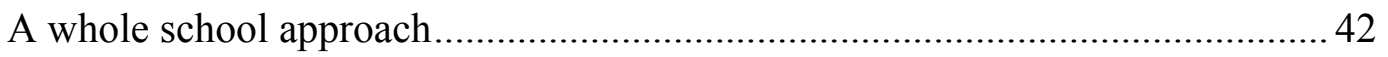

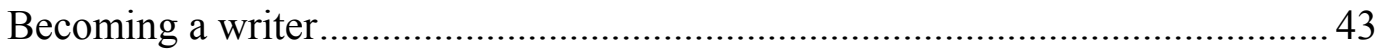

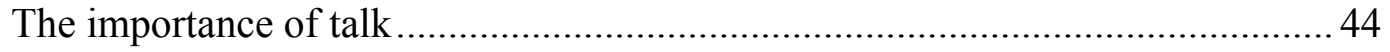

Classroom approaches to support boys' writing.................................................... 45

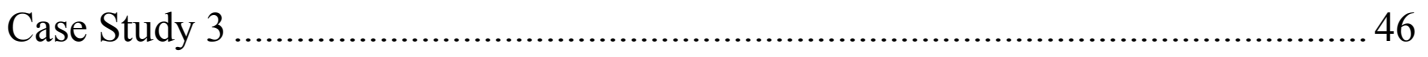

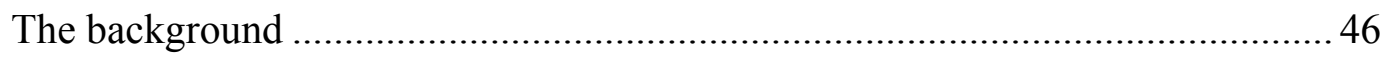

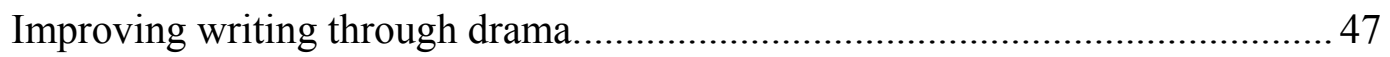

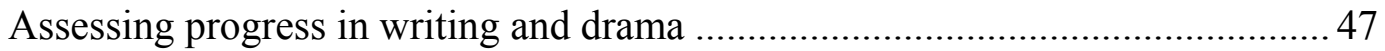

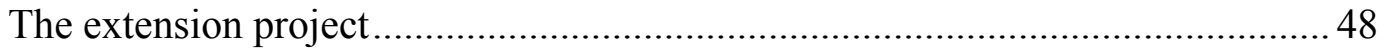

Evidence for drama leading to better writing: effects on writers and writing ...... 49

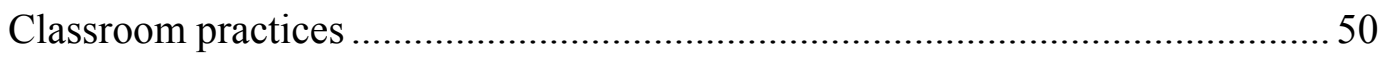

Factors which supported curriculum development: across schools ...................50

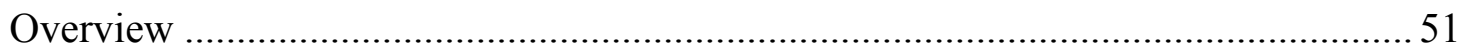

CHAPTER 5: Pedagogy II: Issues about Teaching-Learning Styles.....................53

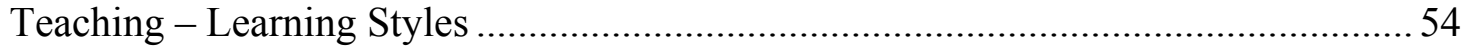

Case Study 1: Accessing Kinaesthetic Learners more effectively .........................5 54

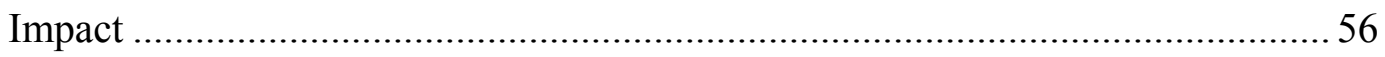

Case Study 2: An approach based around multiple intelligences...........................57

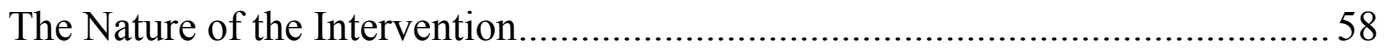

Outcomes in the Originator School: Patterns of Achievement ............................58

Outcomes in the Originator School: Students' Views and Perspectives from the

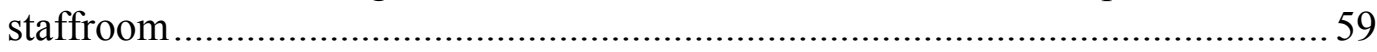

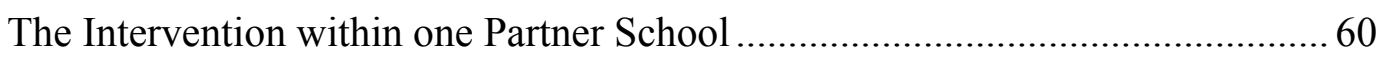

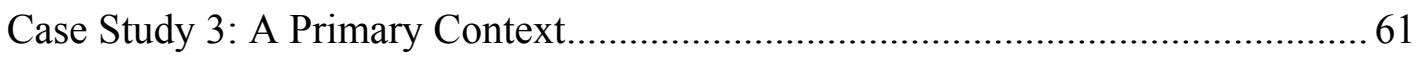

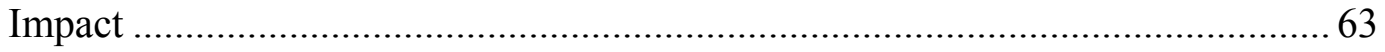

Reflections on Pedagogic Approaches Focusing on Preferred Learning Styles.......... 64

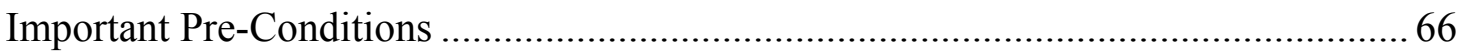

CHAPTER 6: The Context of the Individual: Target-Setting and Mentoring........ 68

Target-Setting and Mentoring within the Raising Boys' Achievement Project........... 68

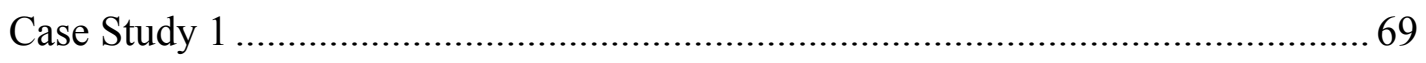


The background: 69

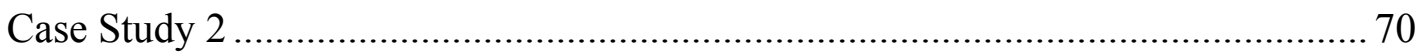

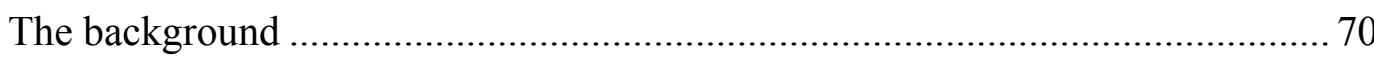

Target-Setting and Mentoring Approaches ........................................................... 72

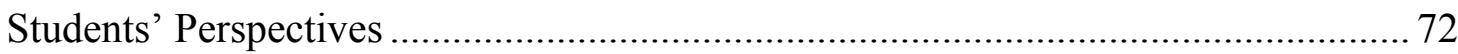

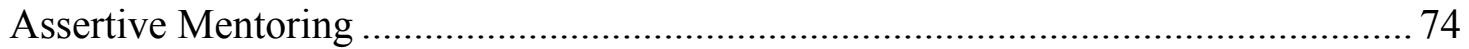

Pre-conditions for Successful Mentoring and Target-setting ................................... 75

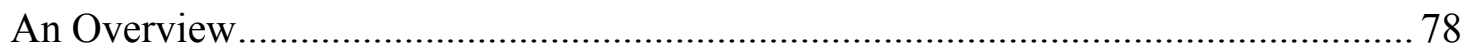

CHAPTER 7: Organisational Contexts - Equal Opportunities in the Single-Sex Classroom ........................................................................................................... 8

Single-Sex Classes in Mixed Comprehensive Schools : the issues........................... 80

Single-Sex Classes within the RBA Project: initial work .......................................... 80

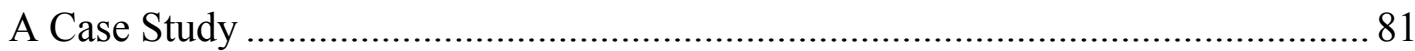

Learning in Single-Sex Classes: the Students' Perspective ............................... 83

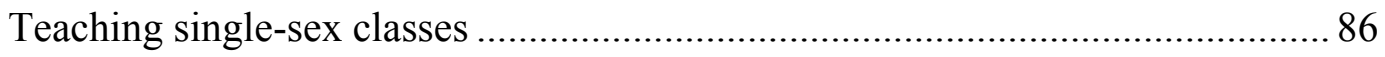

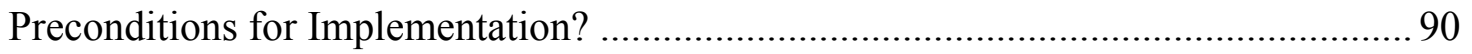

CHAPTER 8: Socio-Cultural Approaches: the Secondary School Context .............92

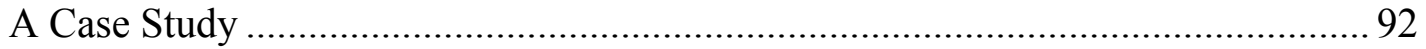

The key leaders / key befrienders scheme ...................................................... 93

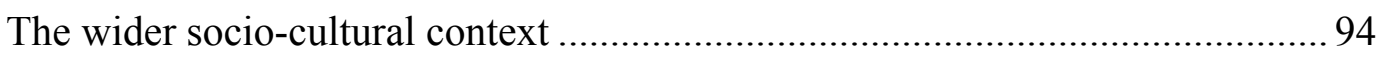

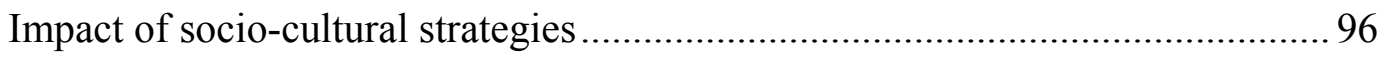

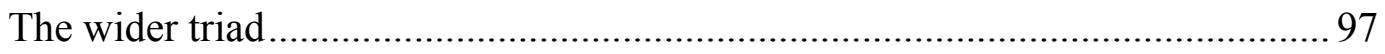

Pre-conditions for Successful Implementation of Socio-Cultural Strategies .............. 99

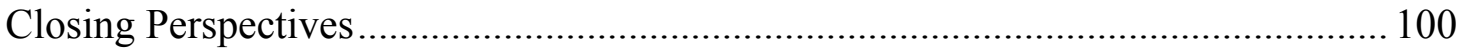

CHAPTER 9: Socio-cultural Approaches: the Primary School Context ............... 102

Socio-cultural Strategies through a Whole-School Approach.................................. 103

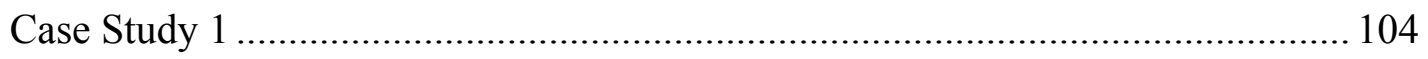

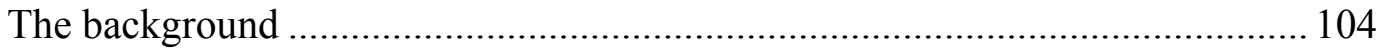

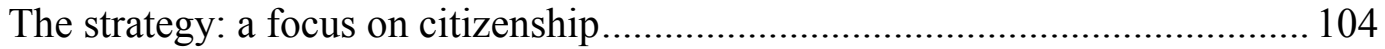

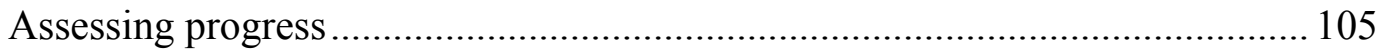

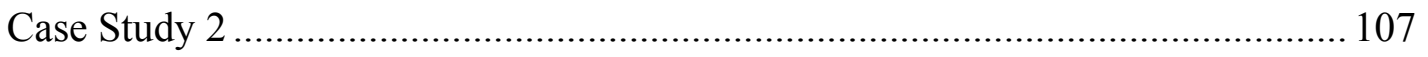

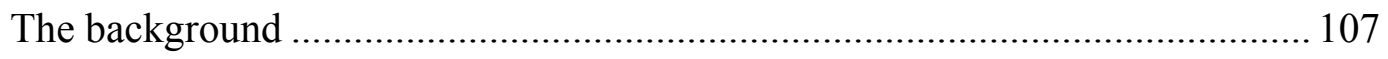

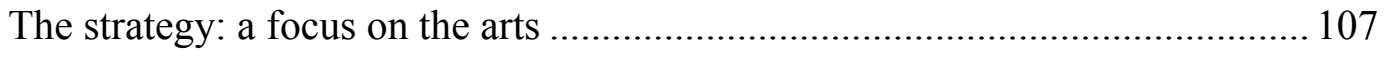


Assessing progress 108

A socio-cultural strategy through a classroom-based approach .................................... 111

Case Study 3

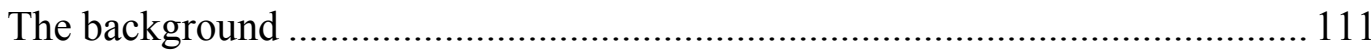

The strategy: peer support through a paired reading scheme .............................. 111

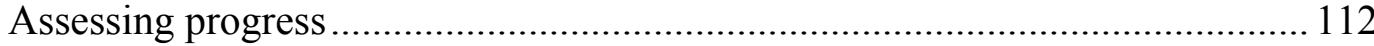

Evidence of changing attitudes across the triads ...................................................... 113

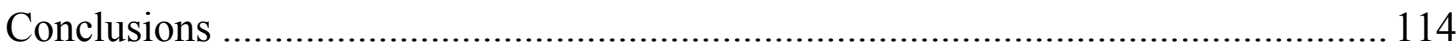

CHAPTER 10: Gender and Achievement in Special Schools .................................. 116

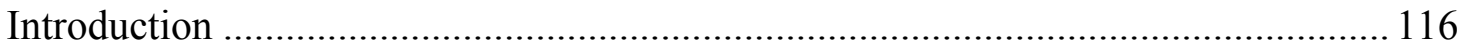

The Research Participants and the Focus of Inquiry ................................................. 117

Gender, Achievement and Special Education: some general findings ....................... 117

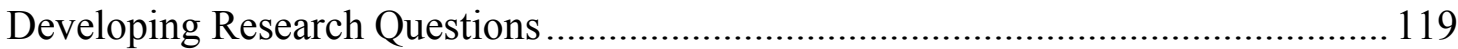

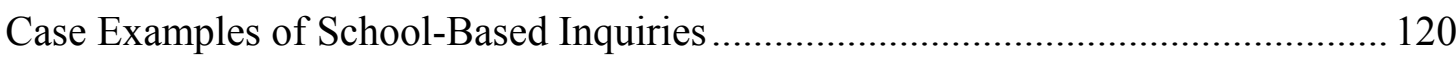

Case Study 1: Teaching and learning English in Years 10 and 11 in the wider context of whole school development ............................................................................ 120

Case Study 2: Boys' self-esteem and engagement in learning ............................... 123

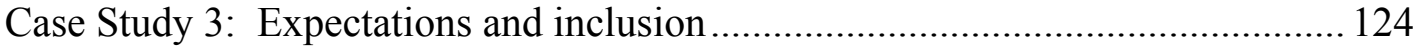

Case Study 4: Supporting concentration and independence in classroom learning 125 Some General Conclusions from the Case Studies................................................... 126

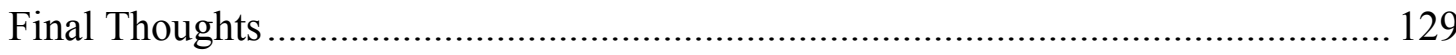

CHAPTER 11: The Broader Concerns: Developing, Supporting and Sharing Innovative Ideas ........................................................................................................................ 131

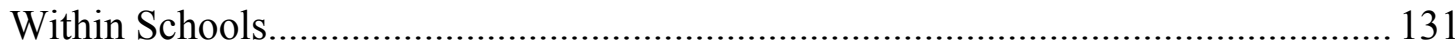

Understanding the context and meeting the preconditions for development...... 131

Factors that impede steady progress ................................................................... 133

The importance of a longterm approach ............................................................ 133

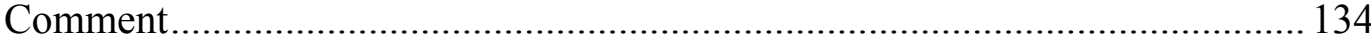

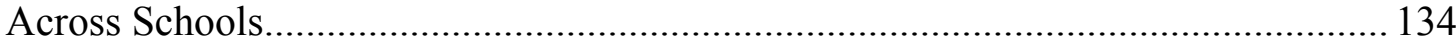

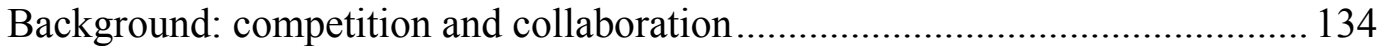

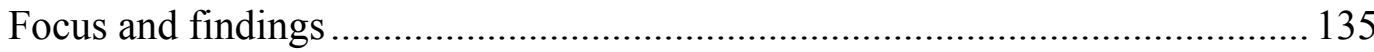

From hierarchy to equality ............................................................................. 135

The benefits of working in a triad ...................................................................... 136

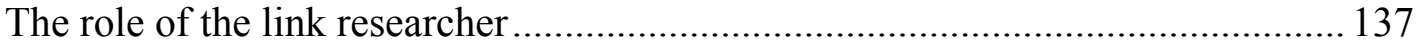




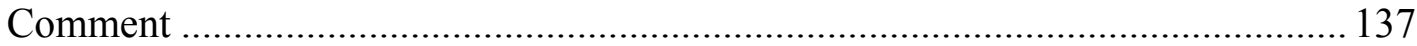

CHAPTER 12: Concluding Discussion........................................................... 139

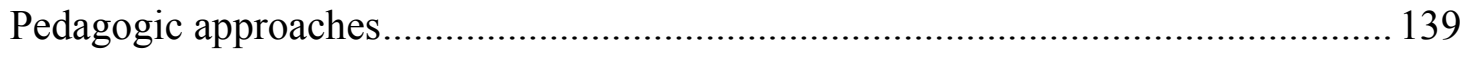

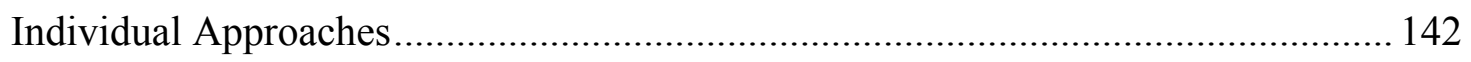

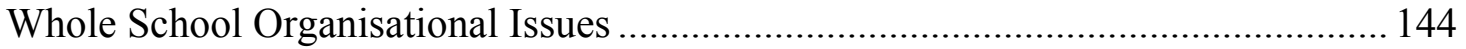

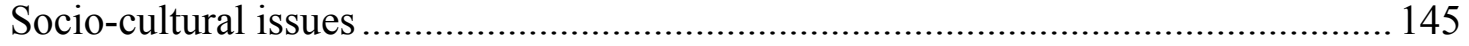

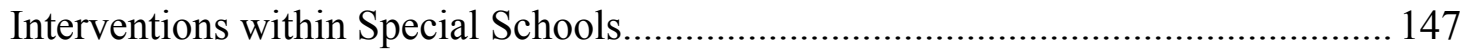

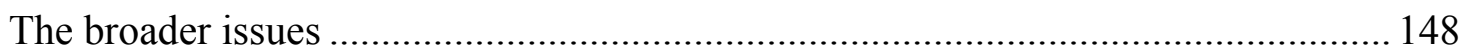

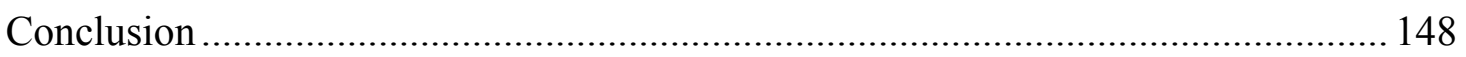

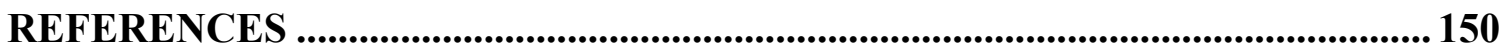

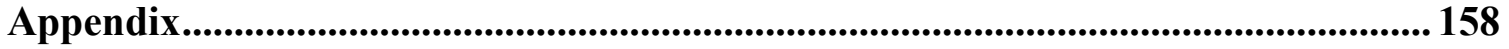

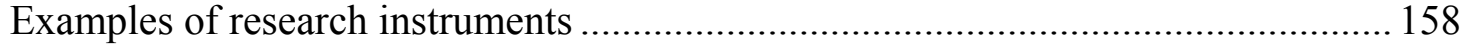




\section{ACKNOWLEDGEMENTS}

The research team would like to thank all those who have worked so persistently and enthusiastically on this project over the period 2000-2004.

In particular we acknowledge our debt to those school students who have shared their views and perspectives with us, with such tolerance and good humour, and to headteachers and school staff who have worked with us over the four-year span of the project.

The research team has been supported throughout by Research Officers at the DfES, to whom we give thanks, and by four part-time Research Assistants who have undertaken fieldwork in schools at various stages of the project: Mary Berry (who sadly died late in 2004), Suzanne Carter, Peter Jackson and Jennifer Reynolds. We are very grateful to them for their support and critical analysis. 


\section{EXECUTIVE SUMMARY}

The 'Raising Boys' Achievement Project' (RBA) was a four-year project (2000-2004) which focused on issues associated with the apparent differential academic achievement of boys and girls at key stage 2 and key stage 4 in schools in England. This report highlights some of the dilemmas which are implicit within the debate, explores different interpretations and perspectives about boys' 'under-achievement', and challenges some common misconceptions.

Working with over fifty primary, secondary and special schools in England over four years, we have endeavoured to identify strategies which appear to have the potential to make a difference to boys' (and girls') learning, motivation and engagement with their schooling, and consequently to raise levels of academic achievement. These strategies have been analysed in different school settings through time, in an attempt to identify their essential characteristics, so that they might be transferred to other schools in similar socioeconomic contexts.

The process of transfer of these intervention strategies has involved schools working together in learning triads (each triad consisting of one Originator School and two Partner Schools), with a total of seventeen triads studied, to introduce, refine and consolidate these strategies. The project team has worked with triads throughout this process, supporting, exploring and analysing the process of innovation transfer. In so doing, we have aimed to clarify further the essential characteristics of each intervention strategy, and to identify essential pre-conditions which appear to need to be in place if the potential of the strategy is to be maximised.

\section{'Boys' 'Under-Achievement' ?}

The debate, about whether, and to what extent, boys under-achieve academically in English schools has been high profile since the early 1990s, and it is clear from national data that there is legitimate concern over the achievement levels of some boys throughout their schooling. Rather more boys than girls fail to achieve level 4 in English national tests at the end of key stage 2; rather more boys than girls fail to achieve the $5 \mathrm{~A}^{*}-\mathrm{C}$ benchmark grades in GCSE examinations taken at $16+$. These patterns of academic achievement are evident in most schools in England.

It is crucial, though, to situate the debate carefully:

- Achievement levels in primary and secondary schools, as measured by national tests at the end of each key stage, are rising through time. In some schools and LEAs, this has widened the 'gender gap', at least in the short term, as girls' performances have 'taken off' at a more dramatic level than those of boys. Overall, however, evidence suggests that the gap has stabilised, against a background of a rising trajectory of achievement for both girls and boys.

- There is diversity of gender constructions which indicate that generalisations about 'boys' and 'girls' conceal as much as they reveal. Many boys continue to achieve extremely well at school, both academically and in community, extra-curricular and sporting fields; equally, there are some girls whose needs are not recognised within schools and who under-achieve. The core of the issue in many schools 
revolves around a minority of pupils, rather than a majority; the 'problem' needs to be carefully contextualised, both in scale and in response.

- Although issues of image and status are crucial in impacting upon boys' notions of masculinity, as they search for acceptability and respectability amongst their peers, there are boys who devise coping strategies that enable them to achieve academically. These boys preserve their positions and their masculinity within the legitimised local cultures whilst at the same time meeting their own high targets.

Nevertheless, the continuing gender gap in key stage 2 English results, particularly in writing, where the performance ratio suggests that less than $80 \%$ of boys perform at the same level as girls, suggests that a stubborn problem remains to be tackled. The situation is mirrored at key stage 4, where the improved performances of girls in science and mathematics have not been matched by a comparable improvement of boys' performances in subjects such as Modern Languages, English and the Humanities.

\section{Intervention Strategies}

Initial research with Originator schools (schools which appeared to have strategies in place which were improving the academic achievement of boys without impacting negatively on girls' performances) suggested that strategies could be grouped into four different areas:

- Pedagogic: classroom-based approaches centred on teaching and learning

- Individual: essentially a focus on target-setting and mentoring

- Organisational: ways of organising learning at the whole school level

- Socio-cultural: approaches which attempt to create an environment for learning where key boys and girls feel able to work with, rather than against the aims and aspirations of the school.

Although this classification was a useful device for analysis and identification of the essence of the different strategies, it is clear that these strategies are not self-contained and independent. As our work in special schools suggested, there must be an integration of different approaches if their impact is to be maximised. It is equally clear, however, that socio-cultural approaches are of central importance if schools are to be successful in challenging images of laddish masculinity and ladettish femininity, and getting peer leaders 'on side' and engaged with their schooling.

\section{Pedagogic approaches}

A main pedagogic approach followed by the RBA project in primary schools focused on literacy, essentially because many boys do less well than girls in reading and particularly in writing. In identifying pedagogy which helps to support pupils' reading and writing, however, it became self-evident that these strategies are most effective within a holistic approach, which assimilates opportunities for reading, writing, speaking and listening into an integrated whole.

Thus the aim in one triad became concentrated not so much on a concern about teaching reading, but on a need to encourage boys to become successful and satisfied readers. This involved having a wide range of texts available to stimulate and sustain pupils' interest and to build confidence through paired reading schemes. Crucially, however, this objective was only achieved when teachers were prepared to take the pedagogic decision to give 
pupils space to talk and reflect about their reading, to share ideas about the text and what was enjoyable in it. When this happened and teachers had the confidence to develop an integrated approach to literacy, the standards of reading of many boys improved markedly, sometimes by twice that expected within national test parameters.

In two other primary triads, developing children's writing was given more prominence. In pedagogic terms, more emphasis was placed upon strategies such as paired and group talk, oral preparation for narrative, hot seating and drama and role play. These classroom approaches encouraged pupils to discuss their story lines more explicitly with each other in collaborative contexts, to explore aspects of character, plot, setting and vocabulary, and to write in role, freed from their immediate surroundings. In both these triads, subsequent analysis of boys' writing showed marked improvements in the pace and structure of their story writing, in their character depiction, in their creation of atmosphere within stories and in their use of dialogue as a narrative device. Significantly, too, the performance of many of these boys in National Curriculum tests showed marked improvements, over and above those expected in value-added terms.

We would suggest that gains can be made in primary literacy, particularly in the levels achieved by apparently under-achieving boys, when:

- a variety of interactive classroom activities are adopted, with a 'fitness for purpose', so that both short, specific focused activities and more sustained, ongoing activities are used, as and when appropriate

- acknowledgement is given to the central importance of talk, to speaking and listening as a means of supporting writing.

- the advantages to be gained through companionable writing with response partners and through group work are recognised

- teachers are prepared to risk-take to bring more creativity and variety to literacy

- more integrated use is made of ICT so that quality presentation can be more easily achieved, and drafts amended with more ease.

The project has also focused, in one primary and two secondary triads, on exploring work related to preferred learning styles, and on associated teaching strategies such as mind mapping, physical and practical activities, role play and creative design activities. A number of caveats about such approaches have emerged from our research. We have found little evidence, for example, to support the notion that the dominant learning style of boys differs from those of girls, and that more boys (than girls) favour kinaesthetic learning. Equally, it is often difficult to analyse classroom activities in terms of specific learning styles because many pedagogic activities engage different modalities.

Nonetheless, this emphasis on teaching and learning styles can be effective when:

- such an approach is implemented carefully and holistically

- the emphasis is placed on developing an understanding, with teachers and students, of how learning takes place, through keynote presentations to teachers and students about different modes and styles of learning

- students understand that, as individuals, they have different learning styles, some of which (e.g. visual, auditory or kinaesthetic) may be more prominent than others, 
but that to be effective learners, they must be able to access different learning styles at different times

- teachers are able to plan lessons which encompass different learning styles, and thus become more creative in their teaching, planning and assessing.

Work on preferred learning styles is misconceived if it simply tries to identify and teach to students' dominances within their learning profiles; such an approach misses opportunities and narrows learning. Rather, it is important to locate any discussion of teaching-learning styles within an ongoing staff development agenda, which addresses explicitly issues of classroom pedagogy, because work on preferred learning styles must be translated into teaching style. In this project, most impact on students' achievement took place when teachers explicitly discussed teaching and learning with students, and made them aware, in a collaborative context, of the ways in which they were trying explicitly to change their teaching styles.

\section{Individual approaches}

Individual approaches, based on a coherent and integrated approach to target-setting and mentoring, have been very important in some schools in transforming and sustaining improvements in achievement. There are challenges in implementing such an approach, particularly in the need to avoid focusing scarce resources on a minority of students, usually boys, who hover around the $5 \mathrm{~A} *-\mathrm{C}$ grade benchmark at GCSE, or around level 4 in key stage 2 English. Equally, some mentoring schemes fail because they become oppressive and demotivating for students, or because mentors do not prioritise the time to give credibility to the process.

Our research with two secondary triads suggests that target-setting and mentoring can be successful when there is mutual understanding and shared commitment to all aspects of the process within a school staff, and a common belief and conviction in the system which is held by teachers and students. There are crucial pre-conditions, we suggest, if students' achievements are to be changed in this way:

- Target-setting needs to be both realistic and challenging, not simply based on historic data within the school, but based upon higher expectations and detailed analysis of contextualized value-added data at the individual level.

- Teachers within subjects departments need time and support on a regular and frequent basis, to set targets for individuals within their classes, and to engage in professional dialogue about learning at the level of the individual child.

- Mentoring needs to be developed within an ethos which accepts that mentors will mediate and negotiate with subject teachers on behalf of 'their' student, and subsequently challenge 'their' student to achieve more.

- The mentor needs to be credible to individuals, collaborative and supportive on the one hand, offering strategies, advice and encouragement, but crucially, also assertive and demanding on the other, so that disengaged students have the opportunity to protect their own image and use their mentor's pressure to excuse their own involvement in academic work.

When these pre-conditions are in place, our experiences during the course of this project confirm that target-setting and mentoring have the potential to change the aspirations and 
engagement of many students, particularly those previously disenchanted and disengaged. Many boys, in particular, have achieved far better than predicted on the basis of previous performances, because they have developed a sense of self-belief, and come to realise that they can reconcile academic work with the self-image which they wish to promote.

\section{Whole school organisational approaches}

Within the area of whole school approaches, we have focused on single-sex classes as a mode of organization in co-educational schools. There is emerging evidence, despite the reservations of those who feel that comprehensive schools should be co-educational in all respects, that many girls and boys feel more at ease in such classes, feel more able to interact with learning and to show real interest without inhibition, and often achieve more highly as a result.

As with other intervention strategies, however, there is the need for some caution in any analysis. Such single-sex classes are not a panacea in themselves; in some schools, boys'only classes have become very challenging to teach, or stereotyping of expectation has established a macho regime which has alienated some boys. Even in the most successful schools, both boys and girls have consistently said that they do not want to be in single-sex classes for all lessons.

Evidence in favour of the development of single-sex classes for some subjects, from both students' voices and from an analysis of levels of academic achievement, is nonetheless persuasive. Again it has been possible, through an examination of good practice, to identify a series of pre-conditions for successful implementation. These include:

- The use of a proactive and assertive approach in the classroom, which avoids the negative or confrontational, conveys high expectations and a sense of challenge, and uses praise regularly and consistently.

- The development of a team ethic, to establish a class identity, supported by humour and informality on the part of both teachers and students, to identify with their interests and enthusiasms, but without reinforcing stereotypes.

- Senior managers who give high profile and active support to single-sex classes, and see them as a central plank within the achievement ethos of the school, rather than simply allowing them as an 'experiment' which might succeed or fail.

- Promoting the intervention actively to governors, parents and carers, and all staff, so that single-sex classes can be promoted and sustained through time.

Where these pedagogic and organizational pre-conditions have been in place, in selective but carefully targeted subjects for specific students, there has been a positive effect on achievement, particularly in relation to boys' performances in modern languages and English and girls' performances in sciences and mathematics.

\section{Socio-cultural approaches}

It is self-evident, not only in secondary, but also in primary schools, that some boys go to considerable lengths to protect their macho image and their sense of self-worth by indulging in a range of non-conformist behaviour which frequently prevents them, and others in the same classes, from achieving well. Such disruptive behaviour, seeming lack of effort and apparent disengagement has the effect, too, of protecting such boys from 
possible failure. These boys are frequently key players in affecting the tone and engagement of the whole year group, and on occasions they hold considerable sway amongst their peers, both male and female.

Schools which have successfully addressed these challenges have adopted a range of socio-cultural strategies to integrate these boys more fully within school life. During the project, we have worked with, and evaluated, a number of such strategies:

- Citizenship initiatives in primary schools, linked to Schools Councils, teambuilding clubs, circle time and a 'You Can Do It' programme.

- A central focus on the Arts across primary schools, with artists-in-residence schemes, poetry weeks, dance sessions run by professional dancers, and drama productions which allocated lead roles to disengaged boys.

- Paired reading schemes between year 3 and year 5 pupils, with the explicit rationale of promoting self-esteem amongst the year 5 'experts'.

- A key leader and key befriender scheme in secondary schools, targeting and supporting particularly those students (usually more boys than girls) whose physical presence, manner and behaviour exerted considerable power and influence within the peer group.

These intervention strategies have been challenging to transfer to Partner schools. In the secondary contexts, the key leader / key befriender scheme has only been successful where:

- it has been possible to identify accurately the key leaders in the year group, who will also respond positively to initiatives the school puts in place

- key befrienders are willing to work with disengaged and challenging individual students, and who are credible, able to establish rapport, use persuasion and model non-stereotyping attitudes and behaviour

- the school has been able to mould expectations and change aspirations, through the creation of a school 'house' style, with emphasis on uniform, on regular attendance and responsive behaviour monitoring, and on the school day as a time of learning rather than social activity.

In the primary contexts, achievement and aspirations have been transformed in those schools where:

- headteachers acknowledged under-achievement and used familiar curricular activities creatively and imaginatively to target it

- teachers were willing to take risks to engage individual pupils in roles where they were actively supported to make choices and to achieve success

- staff were fully committed to create opportunities to give pupils space to articulate their feelings and emotions

- pupils were offered challenge but also activities which individually they could excel in.

When these preconditions are in place, it has been possible to generate a sense of inclusiveness for under-achieving students, improving their engagement, developing an 
increased sense of responsibility, and contributing to an increasing sense of confidence and positive self-image, as well as enhancing academic achievement.

\section{Interventions within Special Schools}

Many of the intervention strategies developed within the context of special schools are similar to those developed in the mainstream context. These included:

- an integrated approach to boys' writing, through visual and aural approaches, and scaffolding of tasks

- the development of pedagogy which took account of work on learning styles and active learning

- evolving whole school approaches which responded effectively to pupils with low self-esteem, and their developing disaffection because of a placement in special schools which separated them from their peers at primary-secondary transfer.

Two differences in emphasis, however, were:

- in the context of small classes, an overriding concern with the social and personal well-being of the individual child, as well as with their academic achievement

- the need for tight integration of different approaches (pedagogic, individual, organizational and socio-cultural) within a holistic whole school framework .

Despite these differences, the evidence emerging from the project does not support the case for a separate special education pedagogy, but rather for an integration of approaches to develop inclusive pedagogy.

\section{The Broader Issues}

Whilst preceding discussion has focused on specific strategies, broader findings also emerged from the project.

The triad structure allowed schools to work positively together in a non-hierarchical relationship, to evaluate, refine and develop different strategies, to transfer them to other contexts and to share good practice.

The project also highlighted the importance of school and community contexts, where there are different routes to achievement, and alternative ways forward. Successful strategies emerged that schools found appropriate to their own particular contexts; these were implemented through collaboration, rather than through imposition.

In addition to the pre-conditions necessary for the successful development of specific strategies, we identified a further set of more generic preconditions necessary before any strategy could succeed. These included leadership support and commitment by all staff, a clearly articulated ethos where high expectations were the norm, a culture which celebrated achievement in its widest sense, and an emphasis on pedagogic practice.

\section{Conclusion}

We are confident that these intervention strategies, developed by participating schools in contrasting socio-economic environments across England, can be effective in raising boys' achievement. 
Such strategies also have the potential to raise girls' achievement, and so in many instances the gender gap - at least in the short term - is perpetuated. We are not unduly concerned about this, since we do not find it acceptable to promote intervention strategies which, whilst supporting boys' learning, are detrimental to girls in either an academic or a social sense.

A recurring theme in these policy initiatives, whether related to pedagogy, forms of organisation or strategies which focus on the individual, is the fundamental importance of context and of whole school approaches. In each case, however, the strategies are no panacea: they cannot be implemented successfully without regard to the necessary preconditions which we have explored in the main report.

In addressing issues of under-achievement it is crucial that intervention strategies address issues linked to students' attitudes and image, their expectations and aspirations, tackled at the core. To be fully effective, these strategies must be developed systematically through time, and subsequently evaluated and refined in the light of experience. We have no evidence to suggest that short-term strategies are likely to impact positively upon students' achievements in sustainable and ongoing ways.

Finally, our research does not support the notion that there is a case for boy-friendly pedagogies. Pedagogies which appeal to and engage boys are equally girl-friendly. They characterise quality teaching, and as such are just as suitable and desirable for girls as for boys. 


\section{CHAPTER 1: The Context of the Project}

The apparent academic 'under-achievement' of boys at school has been a pervasive theme in both academic and public debate over the last ten years. In the United Kingdom, the increasing availability of national performance data has focused the debate on the 'gender gap' between the level of boys' and girls' performance, whether at the age of 7 in reading and writing or at the age of 16, in virtually all GCSE subjects. Each summer, media reports from the mid 90s onwards have focused on 'lost' boys (Gold, 1995), referring to boys 'being lapped by girls' (Williams, 1995), emphasising the need to 'rescue Britain's lost boys' and to devise 'rescue plans for the weaker sex' (Lee-Potter, 2003). This media concern has been reinforced, at different times, by prominent figures within the educational establishment, with Her Majesty's Chief Inspector declaring that the failure of boys was 'one of the most disturbing problems we face in the whole educational system' (Woodhead, 1996), and the then Secretary of State for Education maintaining that

'We face a genuine problem of under-achievement among boys, particularly those from working class families. This under-achievement is linked to a laddish culture which in many areas has grown out of deprivation, and a lack of both self-confidence and opportunity'. (Blunkett, DfEE, 2000).

Similar concerns were voiced in other Western economies, with talk in Australia of 'underachieving and under privileged' boys (Gilbert \& Gilbert, 2001; Martino \& Berrill, 2003) and of boys as the new disadvantaged (Kenway et al, 1998; Martino \& Meyenn, 2002). In the United States, too, there emerged concern around the theme of how to 'protect' boys, and on how teachers, counsellors and therapists might identify and respond to boys' hidden despondency and depression. The educational scene in mainland Europe echoed similar concerns: in Belgium, research suggested that boys' culture was less studyoriented than girls' and that this impacted upon achievement levels in secondary schooling (Van Houtte, 2004); in Sweden, there has been a concern with the need to develop boys' social competence and democratic understanding (Ohrn, 2001); in Germany girls have been obtaining better school marks than boys, repeating classes less often and gaining school certificates more successfully.

In the United Kingdom, this concern with boys' academic achievements and with their engagement with schooling embodied a marked change in emphasis within the gender mainstreaming debate. During the 1970s and 1980s, the focus of work on gender and equal opportunities was centrally on girls. Research demonstrated that career expectations and subject choices were structured along traditional gender lines, to the disadvantage of girls (Sharpe, 1976; Deem, 1980; Griffin, 1985). They showed that aspects of the hidden curriculum contributed to the reinforcement of sex roles (Woods, 1990) and that girls were frequently marginalized in the classroom, with teachers responding more readily to boys who monopolized linguistic and physical space and teacher attention (Mahony, 1985; Stanworth, 1987). Equal opportunity initiatives focused on confronting these issues, on new textbooks and language conventions to reduce gender bias, on analysing classroom dynamics and interactions, on a common curriculum to attract more girls into science, technology and mathematics (Myers, 2000), and on policies to reduce gender discrimination in career structures (Rudduck, 1994).

The debate about boys' 'under-achievement' focused the concern on to boys in their own right, rather than simply as students whose attitudes and approaches had to be changed if 
female disadvantage was to be eliminated (Kenway, 1997). There is an increasing unease with some aspects of this changed focus (Epstein et al, 1998; Francis \& Skelton, 2001; Martino \& Berrill, 2003) and the sense of moral panic which this has induced in some commentators and governments. We share this unease, not least because the notion of male disadvantage is one which is difficult to sustain in the labour market of Western economies and in the wider context of our male-oriented society, and there is evidence to suggest that in some schools girls still remain invisible and teachers gender-blind (Warrington \& Younger, 2000).

Nevertheless, there is legitimate concern over the achievement levels of some boys, and it is apparent that in some schools, more boys are likely to perform below their potential, as defined in value-added terms, than girls. This differential pattern of achievement poses a major challenge for those concerned with raising standards in schools. Work in individual school contexts (Rudduck, Chaplain \& Wallace, 1996; Pickering, 1997; Younger et al, 1999) emphasises that more boys than girls are disengaged, that more discipline problems are perceived to be caused by boys, that more boys are excluded from secondary schooling. Similarly, more sophisticated attempts at value-added analysis (DfES, 2004a) and of charting the progress made by girls and boys who achieved similar levels of achievement at the ages of 7,11 and 14 (as measured in National Curriculum tests and teacher assessments), suggest that more girls than boys make significantly greater progress over the course of key stages 2- 4 in English primary and secondary schools.

\section{Why Differential Achievement?}

Previous research has been carried out at a number of levels in an attempt to explain the failure of boys to achieve at the same levels as girls, and debate about the reasons for boys' lower levels of achievement and, crucially, possibly ways of narrowing the gap, has been vigorous. A variety of different explanations have been offered, and the gender gap is variously construed as resulting from:

- brain differences between girls and boys (Sommers 2000, Gurian, 2001), with links to boys' testosterone and the 'natural' development of boys (Biddulph, 1998). Similarly, Archer \& Lloyd (2002) have argued for a biological construction of masculinity, citing studies which show behavioural sex differences at a very early age, before children are able to form any notions of socially constructed gender (Connellan et al, 2000; Baron-Cohen, 2003);

- boys' disregard for authority, academic work and formal achievement (Harris et al, 1993; Rudduck et al, 1996), and the formation of concepts of masculinity which are in direct conflict with the ethos of the school (Connell, 1989; Mac an Ghaill, 1994);

- differences in students' attitudes to work, and their goals and aspirations (Younger and Warrington, 1996, Warrington and Younger, 1999), linked to the wider social context of changing labour markets, de-industrialisation and male unemployment (Arnot et al, 1998);

- girls' increased maturity and more effective learning strategies (Boaler, 1997; Gipps, 1996), with the emphasis on collaboration, talk and sharing (Askew \& Ross, 1988; Fennema, 1996), whilst boys were seen neither as competitive nor as team players, unwilling to collaborate to learn (Barker, 1997), and less inclined to use 
cooperative talk and discussion to aid and support their own learning (Gipps, 1996);

- differential gender interactions between pupils and teachers in the classroom (Younger et al, 1999).

Crucial to this discussion, however, is the need to understand how important it is for many boys to be accepted by other boys, to enable them to identify with and act in line with peer group norms, so that they are seen as belonging (Skelton, 2001; Martino \& PallottaChiarolli, 2003) rather than as different. Such acceptance is often dependent on an act, negotiating an acceptable identity, and incorporating aspects of laddishness of behaviour and risk-taking (Jackson 2002, 2003). Expressed in behaviour, speech, dress code and body language, such laddishness often runs counter to the expectations of the school, but such behaviour is seen as a reasonable cost by boys if it allows them to protect their macho image, and enable them to ensure their acceptance as part of the chosen group. (Francis, 2000).

Our research in many English schools over the last decade has reiterated this concern with image and the extent to which social groupings plays a major role in students' lives. Boys in schools in very different socio-cultural contexts, in inner cities and in rural counties, in Southern England's commuter belt and in Northern England's former mining villages, have all stressed this common theme of the vital need to conform to peer pressure, to be part of the crowd and to live up to crowd norms and expectations. Unlike girls, whose interests are quite widely spread, boys' groups mainly revolve around a football culture, and boys with little or no interest in football are often excluded or marginalized (Swain, 2000; West, 1996). Some boys, particularly those in higher sets, certainly are part of a group where hard work is accepted, and others have learnt to take no notice of taunts from their peers, but for many boys, being 'one of the lads', being 'real hard', 'having a laugh sometimes', 'not showing your emotions and having to win', embody the essence of the all-important macho image (Mac an Ghaill, 1994).

The adoption by lads of specific strategies associated with laddishness also minimises the possibility of failure and the consequent loss of status and esteem in the group context; it is linked to an avoidance of the feminine and the perceived 'stigma' of homosexuality (Jackson, 2002).

\section{Which Boys? Which Girls?}

As the debate has intensified in the United Kingdom, so it has become obvious that the issue of boys' 'under-achievement' is far more complex and multi-faceted than assumed by some commentators. While it is clear that many boys negotiate a position with respect to the locally dominant masculinity, which preserves their image and status and leads them to take pride in disengagement with school, some boys also devise coping strategies which enable them to achieve academically within a legitimised local culture. Not all boys are under-achievers, therefore, and the issue of 'under-achievement' does not affect all boys. An all-pervasive view of boys as under-achieving because of a laddish masculinity ignores the fact that, in many schools, boys are achieving high levels of success in academic, community, sporting and artistic contexts. Indeed, many boys have always done extremely well, and continue to do so (Arnot et al, 1998). Equally, there are those boys who define their sexuality differently from the 'mainstream' macho, football-loving boys: gentle 
caring boys who find their comfort zone in the company of girls and women (Mac an Ghaill 1994, Martino and Pallotta-Chiarolli 2003). Whilst there are boys who can be aggressive perpetuators of homophobic aggression against other boys, not all boys act in the same way.

Just as it is important to look beneath the stereotype of the 'normal' boy, and acknowledge multiple perspectives on masculinity, so there are different kinds of girls and multiple perspectives on femininity (Frosh et al, 2001, Reay 2001). Not all girls are highachievers and conform to the conscientious, hard-working and well-motivated stereotype, distracted from their endeavours by recalcitrant boys. Indeed, some girls are taking on the 'laddish' attributes of their male peers (Jackson, 2004), and we need to pay greater attention to the monitoring of withdrawn, quiet, 'less visible' girls, whose quietness may hide severe problems (Bell, 2004). Boys do not have a monopoly on such matters: in many schools, there are also disengaged girls who do not reach their potential academically.

Whilst our main focus in the Raising Boys'Achievement Project has been on gender, we have also tried to take account of the complex intersections with ethnicity and class (Sewell, 1998; Gillborn \& Mirza, 2000), and we acknowledge explicitly the privilege which advantages some boys over others, and over some girls, and recognise that this usually reflects, at least in the United Kingdom, social class factors (Epstein et al, 1998). Indeed, our selection of project schools has reflected the crucial role of different ethnic and social class backgrounds. This has inevitably added to the complexity of the research, and made analysis more tentative and multi-faceted, but such is reality.

It is inappropriate, therefore, to generalise uncritically about girls and boys: issues of ethnicity and class, of individuality and sexual inclination, differing images of femininity and masculinity, all affect motivation, attitude and achievement. The emphasis has to be placed upon variety and plurality, more than upon similarity and uniformity. Student interviews themselves reveal that girls and boys often feel uneasy and express disquiet when notions of sameness are attributed to them.

At its simplistic level, then, the 'boys' under-achievement' debate ignores the diversity of gender constructions which exist within the schools and societies in which boys and girls operate. Nevertheless, whilst it is nonsensical to accept the simplistic view that the issue is to do with the under-achievement of most boys (Arnot et al, 1999), our own research, particularly interviews with hundreds of boys over the last decade, has shown that there are typical patterns of behaviour to which many boys conform. Gillborn and Mirza's research, too, has shown that - when educational performance of boys and girls is compared within social classes, or within ethnic groups - girls as a group invariably do better than boys as a group (Gillborn and Mirza, 2000). There is also evidence to suggest (Warrington and Younger, 1999) that more girls achieve top grades in their school-leaving examinations than do boys. In the light of this, we think that it is admissible to recognise that, although boys are not an undifferentiated group, there are broad similarities within sub-groups which allow us to make valid generalisations, and within this, if we compare similar groups of boys with similar groups of girls, we can confidently point to evidence of lower levels of academic attainment by boys. 


\section{CHAPTER 2: The Gender Gap: Dilemmas and Debates}

\section{The Nature of the Gender Gap}

The education system in the United Kingdom throughout the 1990s was characterised by a concern with the improvement of standards, on identifying patterns of achievement within the context of 'value-added' measures, and on the compulsory testing of students in English, mathematics and science at 7, 11, 14 and 16. This data-rich environment highlighted yet further the extent to which disparities existed within the educational system, within contexts of class, ethnicity and gender. Whilst there is dispute, as we show later, about the extent to which the gender gap is narrowing, stabilising or widening (Gorard et al, 1999, 2001), it is undeniable that there is, in many schools, differential achievement between girls and boys (Arnot et al, 1998; Tinklin et al, 2001; Younger et al, 2002; Warrington et al, 2003).

In primary schooling, these differences in achievement are quite narrowly defined. At key stage 1, results of the 2004 National Curriculum tests (Table 2.1) show an 8 percentage points difference between girls and boys who achieved level 2 or above in reading and an 11 percentage points gap in writing, a pattern that has been reasonably consistent through time. Data for Mathematics, in contrast, show little difference between the percentage of girls and boys achieving level 2.

\section{Table 2.1: Key Stage 1 Performance Profiles 2000-2004 (Autumn Packages, DfES)}

\% boys and girls achieving level 2 or above in national tests

\begin{tabular}{|c|c|c|c|c|c|c|}
\hline \multirow{2}{*}{ Reading } & \multicolumn{2}{|c|}{2000} & 2001 & 2002 & 2003 & 2004 \\
\cline { 2 - 7 } & Boys & 79 & 80 & 81 & 80 & 81 \\
\hline \multirow{2}{*}{ Writing } & Girls & 88 & 88 & 88 & 88 & 89 \\
\cline { 2 - 8 } & Boys & 80 & 82 & 82 & 76 & 76 \\
\hline \multirow{2}{*}{ Mathematics } & Goys & 89 & 90 & 90 & 87 & 87 \\
\cline { 2 - 8 } & Girls & 91 & 92 & 92 & 91 & 92 \\
\hline
\end{tabular}

Similarly, national data over the period 1996-2004 reveal that although there has been little difference between the percentages of boys and girls achieving level 4 or above in the National Curriculum key stage 2 (Table 2.2) tests in Mathematics and Science, there has been a marked disparity between the attainment of boys and girls in English. Here, girls have persistently outperformed boys in both reading and writing, although it is also significant that there is a persistently lower levels of boys' and girls' performance in writing than in reading. It should not be overlooked, however, that the percentage of both 
girls and boys achieving level 4 in these national tests in all three subjects rose steadily over time through to 2000 , by around 20 percentage points in most subjects, although there was then a period of relative stability in performance until 2004.

Table 2.2: Key Stage 2 Performance Profiles 1996-2004

$\%$ boys and girls achieving level 4 or above in national tests

\begin{tabular}{|c|c|c|c|c|c|c|c|c|c|c|}
\hline & & 1996 & 1997 & 1998 & 1999 & 2000 & 2001 & 2002 & 2003 & 2004 \\
\hline \multirow{2}{*}{ English } & boys & 50 & 57 & 57 & 65 & 70 & 70 & 70 & 70 & 72 \\
\hline & girls & 65 & 70 & 73 & 76 & 79 & 80 & 79 & 80 & 83 \\
\hline & & & & & & & & & & \\
\hline \multirow[t]{2}{*}{ Reading } & boys & & & & & 80 & 78 & 77 & 78 & 79 \\
\hline & girls & & & & & 86 & 85 & 83 & 84 & 87 \\
\hline & & & & & & & & & & \\
\hline \multirow[t]{2}{*}{ Writing } & boys & & & & & 48 & 50 & 52 & 52 & 56 \\
\hline & girls & & & & & 63 & 65 & 68 & 69 & 71 \\
\hline & & & & & & & & & & \\
\hline \multirow[t]{2}{*}{ Mathematics } & boys & 54 & 63 & 59 & 69 & 72 & 71 & 73 & 73 & 74 \\
\hline & girls & 54 & 61 & 58 & 69 & 71 & 70 & 73 & 72 & 74 \\
\hline & & & & & & & & & & \\
\hline \multirow[t]{2}{*}{ Science } & boys & 61 & 68 & 70 & 79 & 84 & 87 & 86 & 86 & 86 \\
\hline & girls & 63 & 69 & 69 & 78 & 85 & 88 & 87 & 87 & 86 \\
\hline
\end{tabular}

This gender gap persists and widens in the secondary school context. When girls and boys are tested at the age of 14 , at the end of key stage 3, for example, there is frequently around a 15 percentage point difference commonly recorded in achievement levels at National Curriculum Level 5(+) in English and in many of the Humanities, Languages and Creative Arts subjects, although again the difference in mathematics and science is much smaller (Table 2.3).

The academic attainments of boys and girls in their GCSE examinations, taken at the end of compulsory schooling at the age of 16, reveal similar patterns of disparity at school, local education authority and national level. Data for the period 1975 - 1995 (Fig.2.1, adapted from Arnot et al, 1998)) show three distinct stages:

- In GCE / CSE examinations, a period of stability and closeness to parity from 1975 through to the introduction of GCSE examinations in 1988. Patterns of examination entry denied many pupils access to the higher grade equivalence of the GCE examinations, so that fewer students were involved in such final examinations, and only $31.7 \%$ of girls and $28.2 \%$ of boys of the national cohort achieved five or more A-C (or grade 1 CSE) grades in the last year of examination of GCE / CSE (1987). 
- The introduction of GCSE examinations and of a National Curriculum in England and Wales coincided with a period of very rapid change, with the relative difference in girls' and boys' attainments exacerbated. The gender gap increased from 3.5 percentage points in 1987 to 8 percentage points in 1990, when $38 \%$ of girls and $30 \%$ of boys achieved the $5 \mathrm{~A}-\mathrm{C}$ benchmark grades.

- A period of increased stability and continuing disparity, 1991-95, with a gender gap in the region of $8-10$ percentage points between the attainments of girls and boys, together with rising achievement, so that in $199548 \%$ of girls and $38 \%$ of boys achieved the $5 \mathrm{~A}^{*}$-C benchmark grades.

Table 2.3: Key Stage 3 Performance Profiles

$\%$ boys and girls achieving level 5 or above in national tests

\begin{tabular}{|c|l|c|c|c|c|c|c|c|c|}
\hline & & 1997 & 1998 & 1999 & 2000 & 2001 & 2002 & 2003 & 2004 \\
\hline \multirow{2}{*}{ English } & boys & 46 & 57 & 55 & 55 & 57 & 59 & 62 & 64 \\
\cline { 2 - 11 } & girls & 66 & 73 & 62 & 73 & 74 & 76 & 76 & 77 \\
\hline \multirow{2}{*}{ Mathematics } & boys & 60 & 60 & 62 & 65 & 64 & 67 & 70 & 72 \\
\cline { 2 - 11 } & girls & 60 & 60 & 62 & 65 & 68 & 68 & 72 & 74 \\
\hline \multirow{2}{*}{ Science } & boys & 61 & 57 & 55 & 60 & 66 & 67 & 68 & 65 \\
\cline { 2 - 11 } & girls & 59 & 64 & 55 & 59 & 66 & 67 & 69 & 67 \\
\hline
\end{tabular}

Fig. 2.1: Changing levels of performance at GCE / CSE or GCSE (1975-95)

Number of boys per 100 girls achieving $5(+) A^{*}-C$ grades

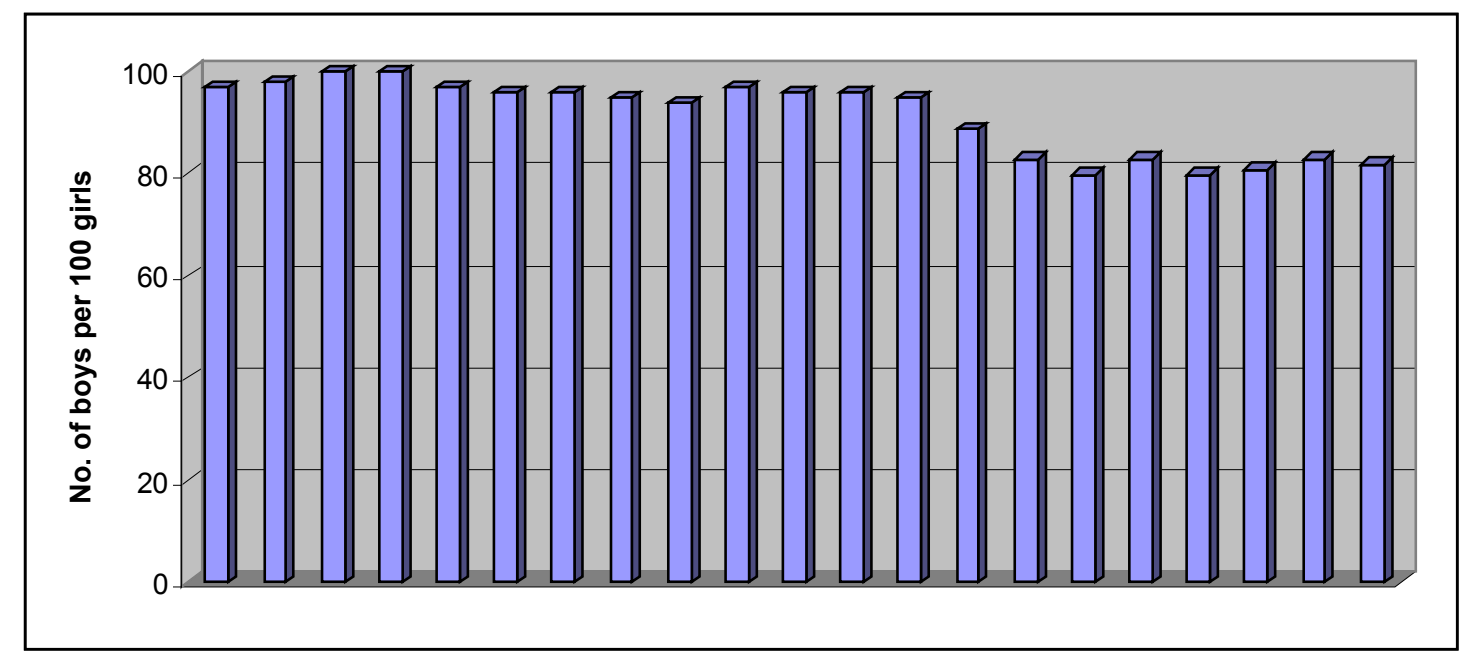

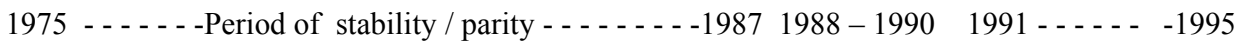

Rapid change Stability / Disparity 
This pattern of stability and disparity has been sustained into the early years of the twentyfirst century (Fig. 2.2). A three-year rolling mean figure for England over the period 19971999 shows that $51.6 \%$ of girls and $41.5 \%$ of boys achieved the benchmark grades (10.1 percentage points difference); by 2004, the gender gap was unchanged, with the benchmark grades being achieved by $58.5 \%$ of girls and $48.4 \%$ of boys (DfES Autumn Package, 2004). Notably, however, this gender gap continues to exist against a background of rising levels of academic achievement of both boys and girls, although this aspect of the national performance profile has been relatively unrecognised and uncelebrated by most commentators.

Fig. 2.2: Percentage entry of girls and boys achieving 5(+) $A$ *-C grades GCSE (or equivalency) 1996-2004

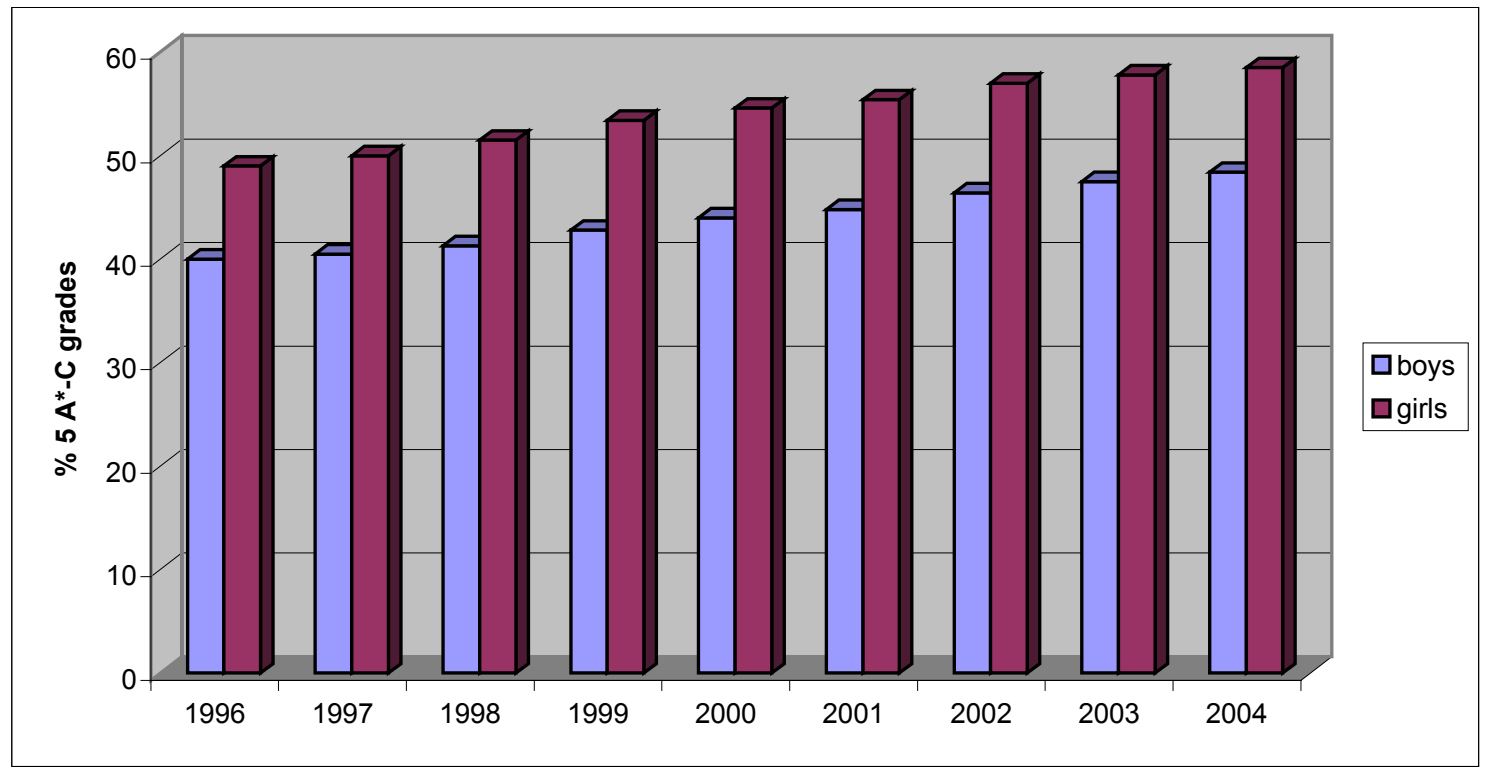

This analysis also confirms, however, that the gender gap at the higher levels of attainment is not growing through time (Gorard et al, 2001), and that it has now stabilised at around 10 percentage points. It is clear also that at the lowest levels of attainment in GCSE terms, there is little evidence of a substantial gender gap; in 2004, for example, $96.6 \%$ of girls and $94.9 \%$ of boys achieved at least $1 \mathrm{~A}^{*}-\mathrm{G}$ GCSE grade or the GNVQ equivalent (DfES Autumn Package data, 2004b).

Another significant aspect of the gender gap at the end of compulsory schooling, however, relates to students' performances in different subjects. Those subjects perceived traditionally as 'boys" subjects, subjects such as Mathematics, Science, Design and Technology, Information Technology, have been colonised by girls with increasing success, whereas boys have failed to engage to a similar degree with traditional "girls" subjects such as the Humanities and Modern Languages.

Thus in 2004, as in every preceding year of the century, girls out-performed boys in every mainstream subject of the National Curriculum (Table 2.4), not only at the benchmark grade level, but (with the exception of Mathematics) also at the highest level of 
achievement at GCSE (i.e. at $\mathrm{A}^{*}$ and $\mathrm{A}$ grade). This confirms the outcome of research we carried out in selective schools in eastern England in the late 1990s, when we concluded that whilst a 'male tail' certainly did exist, there was no corresponding sign of a maledomination of the higher-grade results (Warrington \& Younger, 1999).

Table 2.4: GCSE entries and performance levels, 2004 for subjects taken by over 20\% of national cohort.

\begin{tabular}{|c|c|c|c|c|c|}
\hline Subject & Gender & $\begin{array}{c}\% \mathrm{~A}^{*}-\mathrm{C} \\
\text { grades }\end{array}$ & Gender gap & $\% A^{*}$-A grades & Gender gap \\
\hline \multirow[t]{2}{*}{ Art / Design } & female & 76 & \multirow{2}{*}{20} & 28 & \multirow{2}{*}{14} \\
\hline & male & 56 & & 14 & \\
\hline \multirow[t]{2}{*}{$\begin{array}{l}\text { Design / } \\
\text { technology }\end{array}$} & female & 64 & \multirow{2}{*}{16} & 21 & \multirow{2}{*}{10} \\
\hline & male & 48 & & 11 & \\
\hline \multirow[t]{2}{*}{ English } & female & 67 & \multirow{2}{*}{14} & 18 & \multirow{2}{*}{7} \\
\hline & male & 53 & & 11 & \\
\hline \multirow[t]{2}{*}{ English Lit } & female & 71 & \multirow{2}{*}{14} & 21 & \multirow{2}{*}{7.5} \\
\hline & male & 58 & & 13.5 & \\
\hline \multirow[t]{2}{*}{ French } & female & 58 & \multirow{2}{*}{14} & 21 & \multirow{2}{*}{7} \\
\hline & male & 44 & & 14 & \\
\hline \multirow[t]{2}{*}{ Geography } & female & 66 & \multirow{2}{*}{7} & 26 & \multirow{2}{*}{7} \\
\hline & male & 59 & & 19 & \\
\hline \multirow[t]{2}{*}{ History } & female & 67 & \multirow{2}{*}{5} & 29 & \multirow{2}{*}{5} \\
\hline & male & 62 & & 24 & \\
\hline \multirow[t]{2}{*}{ Mathematics } & female & 53 & \multirow{2}{*}{1} & 11.5 & \multirow{2}{*}{-0.5} \\
\hline & male & 52 & & 12 & \\
\hline \multirow[t]{2}{*}{ Physical education } & female & 61 & \multirow{2}{*}{4} & 22 & \multirow{2}{*}{7} \\
\hline & male & 57 & & 15 & \\
\hline \multirow[t]{2}{*}{$\begin{array}{l}\text { Religious } \\
\text { education }\end{array}$} & female & 71 & \multirow[t]{2}{*}{13} & 32 & \multirow[t]{2}{*}{11} \\
\hline & male & 58 & & 21 & \\
\hline \multirow[t]{2}{*}{$\begin{array}{c}\text { Science } \\
\text { (double award) }\end{array}$} & female & 54 & \multirow[t]{2}{*}{1} & 14 & \multirow[t]{2}{*}{3} \\
\hline & male & 53 & & 11 & \\
\hline
\end{tabular}




\section{Interpreting the Gender Gap: the case for care and vigilance}

This debate on the gender gap and boys' 'under-achievement' is beset by misconceptions, however, and data need sensitive interpretation:

- As Gorard et al (1999) make clear, it is important to distinguish between percentage point gaps and percentage changes in those gaps; thus a change in the percentage of boys achieving the benchmark grades at GCSE, for example from $44 \%$ in 2000 to $48.4 \%$ in 2004 , is a rise of 4.4 percentage points, but a $10 \%$ positive change in the level of boys' performances.

- Similarly, a point difference of 10 points, sustained through time, becomes less significant if the overall levels of attainment rise. This point can best be illustrated by reference to Table 2.5 below, which shows the position in England and Wales over the last decade. Although the gender gap has remained more or less constant (column 4 ), despite the increased proportion of boys reaching the $5+\mathrm{A}^{*}-\mathrm{C}$ grades hurdle (column 3), the ratio between girls' and boys' performances has decreased through time (column 5). Thus the position of boys, whilst still lagging considerably behind that of girls, has improved slowly relative to girls, and the significance of the percentage points gap has reduced.

- We need also to consider examination entry figures of boys and girls, as a proportion of the cohort in the year, as some schools restrict examination access to those students seen more at risk of failure, in order to improve their overall performance data.

Equally, we need to consider the achievements of all students, rather than simply focusing on those who have achieved the high profile benchmark grades, if we are to avoid the risk of establishing an $\mathrm{A}^{*}-\mathrm{C}$ economy (Gillborn \& Youdell, 2000). This involves a recognition, rather than an underestimation, of the achievements of those boys and girls who achieve GCSE grades in the D-G range, and for whom those grades often represent a very real sense of achievement. There have been positive recent developments here, with the publication of performance indicators which place more emphasis upon the average points score per student, using standardised conversion measures across key stages 1-4 and a student's best eight GCSE / GNVQ results to calculate a 'capped points score' (DfES, 2004b), and with the diversification of the scope of the performance indicators to include a greater range of courses and qualifications (DfES, 2004a). Inevitably, though, these developments have taken place within the context of a more sophisticated approach to performance indicators, and this can reiterate the emphasis upon competition and standards. The debate continues to be dominated, at least within the media, by a focus on benchmark grades at GCSE. 
Fig.2.3: GCSE results 1994 -2004: \% Girls and boys gaining 5(+) $A^{*}-C$ GCSEs: Results' profile from a pilot school

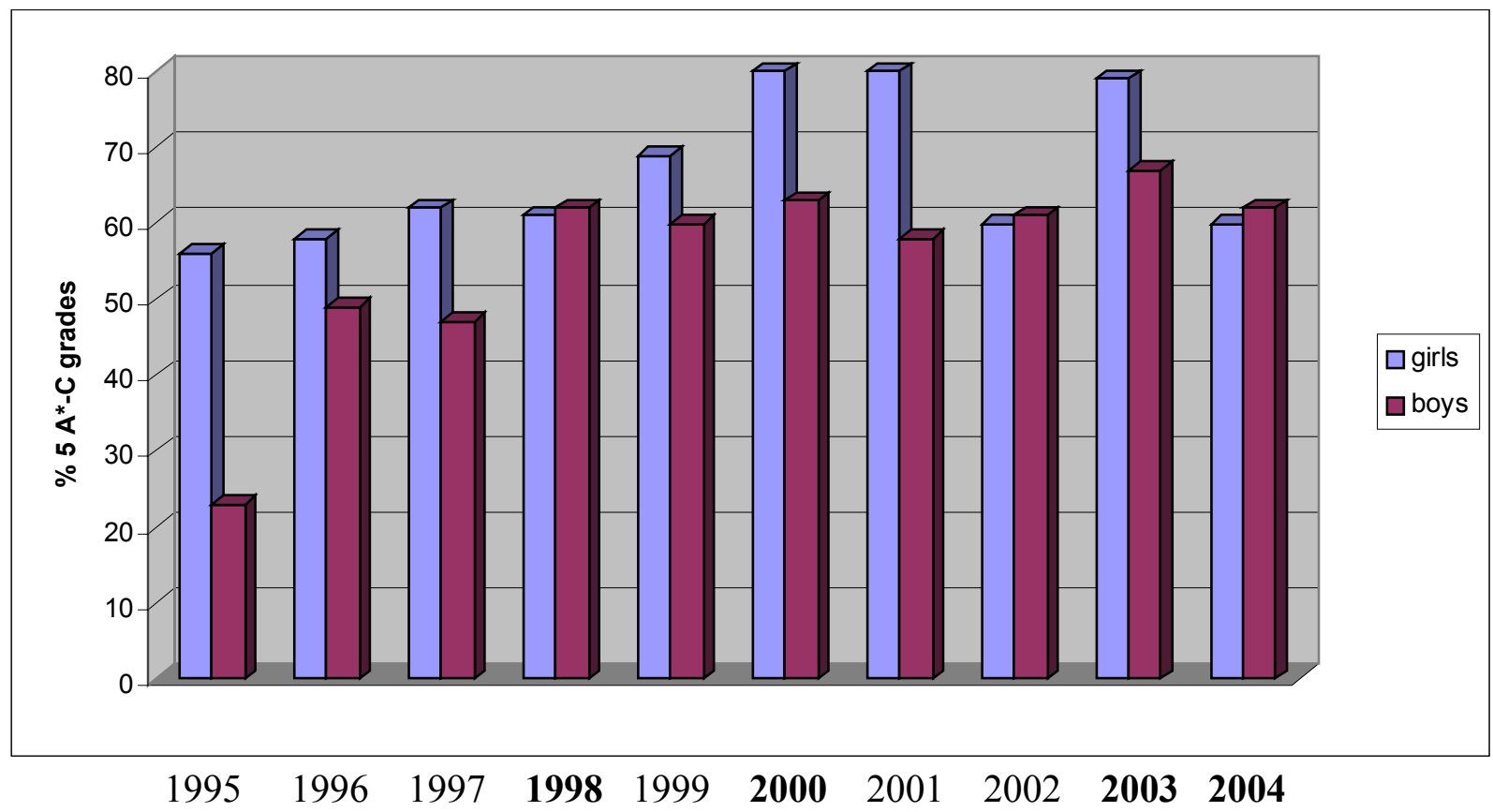

Table 2.5: Percentages of boys and girls reaching the 5(+) $A^{*}$-C grades hurdle, England and Wales, 1995-2004

\begin{tabular}{|c|c|c|c|c|}
\hline & $\begin{array}{c}\text { \% girls achieving 5 } \\
\text { 5(+)A*-C grades }\end{array}$ & $\begin{array}{c}\text { \% boys achieving 5 } \\
5(+) \mathrm{A}^{*} \text {-C grades }\end{array}$ & $\begin{array}{c}\text { Gender gap in } \\
\text { percentage points } \\
\text { (girls - boys) }\end{array}$ & $\begin{array}{c}\text { No of girls reaching } \\
\text { level for each boy } \\
\text { (the ratio) }\end{array}$ \\
\hline 1995 & 48.1 & 38.0 & 10.1 & 1.27 \\
\hline 1996 & 49.4 & 39.9 & 9.5 & 1.24 \\
\hline 1997 & 50.2 & 40.5 & 9.7 & 1.24 \\
\hline 1998 & 51.5 & 41.3 & 10.2 & 1.25 \\
\hline 1999 & 53.4 & 42.8 & 10.6 & 1.25 \\
\hline 2000 & 54.6 & 44.0 & 10.6 & 1.24 \\
\hline 2001 & 55.4 & 44.8 & 10.6 & 1.24 \\
\hline 2002 & 57.0 & 46.4 & 10.6 & 1.23 \\
\hline 2003 & 57.8 & 47.5 & 10.3 & 1.22 \\
\hline 2004 & 58.5 & 48.4 & 10.1 & 1.21 \\
\hline
\end{tabular}


Any debate about the gender gap per se needs careful contextualisation, therefore. As Fig. 2.3 shows, even in large schools with GCSE entries in excess of 300 students each year and stable catchment areas, the pattern of GCSE results can vary markedly from year-toyear. There is a dilemma here: one of our concerns, as a society and as schools, is presumably to narrow and eventually eliminate the gender gap, but in many schools this will be a challenging task. In responding, it is important that we do not become obsessed with the gender gap at the expense of all else. Presumably the senior management in this particular school, for example, ought to be happier with the GCSE outcomes in 2000 than in 1998, and in 2003 than in 2004? An incessant focus on the gender gap must not conceal the fact that it is the absolute achievements of boys and girls that we must be primarily focused on, and that we must locate all our discussions about the gender gap within specific contexts at the individual school level if we are to fully understand achievement profiles within schools.

\section{Concluding Comments}

In summary, there are a number of crucial points which must be emphasised in this discussion of the gender gap:

- It is true that a gender gap continues to exist in many schools in England and Wales, and that in numerical terms there are a significant number of boys nationally who achieve less well than girls of comparable ability.

- But in recent years, the percentage gender gap has decreased slightly as achievement levels have risen.

- There has also been a distinct growth in the number of girls and boys achieving higher level GCSE passes, particularly in terms of girls' achievements in subjects traditionally perceived as boys' subjects', in physical education and design / technology, as well as in science and mathematics.

- But there has been a lack of comparable improvement in the achievement of boys in subjects such as English, Foreign Languages and the Humanities.

- Equally, it is also true that concepts of 'under-achievement' have not been sufficiently clarified throughout this debate; too often, high-achieving students who have under-achieved have not been identified, and those students who have achieved over their apparent potential but at relative low levels of achievement have not been given the praise and recognition which has been their due.

- The use of increasingly more sophisticated value-added measures has enabled schools to make more targeted judgements about the academic performance of individuals and groups of students within their own contexts. This has created its own set of dangers, however, particularly in terms of the targeting and rationing of scarce resources to those perceived to be on the borderlines of benchmark grades at GCSE. 


\section{CHAPTER 3: Intervention Strategies}

\section{The Project's Framework and Approach}

The early stages of the Raising Boys' Achievement Project were concerned with recruiting a pilot group of schools (primary and secondary) where the 'gender gap' had demonstrably been narrowed or eliminated during the last three or four years. Initially, we asked LEA Inspectors, DfES officials and Ofsted inspectors to help us identify such schools. Whilst our concerns clearly coincided with the agenda being pursued at both local and national levels, it was surprising how few schools were identified with any degree of assurance. Discussions suggested that changes may have been achieved in a particular subject area or year group but not in others, or that improvements had been initiated but not always sustained. There were, in short, few confirmed examples where those identifying the school felt that improvements had been initiated across a range of subjects or years groups and sustained through time.

Despite this initial difficulty, it was possible, during the pilot stage of the project, to locate eight schools, four primary and four secondary, which met most of the criteria stipulated by the research team. These were that:

- Each school should be developing an active approach to the issue of 'underachievement', and be able to articulate the impact of that strategy on the changing performance profile within the school.

- There should be some evidence that the gender gap was narrowing consistently through time (in at least three of the four years 1996-2000), as a result of the improvement of boys' performances in external tests at key stage 2 or in public examinations at key stage 4.

- Girls' performances should, at the very least, have been sustained at a stable level for three of the four previous years. Schools where the gender gap had narrowed because of a marked fall in girls' achievements over the period 1996-2000 were not considered for the pilot study.

- The catchment area of the school and the school's intake should be reasonably stable over this period, so that any improvements reflected similar characteristics within the student cohort

- Schools should be located in contrasting socio-economic contexts in different parts of England. Thus the secondary pilots varied from an 11-16 school in a deprived inner-urban authority in North-West England, with a largely white, working-class intake of whom over one-third were eligible for free school meals, to an 11-18 high school serving an extensive rural area in East Anglia, with a number of students from homes where there were low expectations of education. Similarly, the primary pilot schools included a 3-11 primary school in an inner city London borough with a high proportion of pupils from minority ethnic backgrounds and twenty-three different languages represented within the school's intake, a large (480 children) 711 junior school in a deprived authority in the West Midlands, and a small (140 children) rural Church of England primary school in South Cambridgeshire, serving a white, relatively affluent catchment area. 


\section{The Outcomes of the Pilot Study}

In the pilot stage, we were able, working closely with headteachers, class and subject teachers and pupils / students, to identify a number of strategies which schools had been developing through time to address under-achievement and to improve boys' engagement with school. These strategies were wide-ranging and well-documented. In the primary sector, unsurprisingly, pedagogic approaches to literacy were high profile: in each school, library provision and reading baskets had been revamped to provide a wider variety of books (fiction, non-fiction, poetry, plays), and particular emphasis had been placed on gender-friendly texts, to make available reading material which was attractive and enjoyable to boys. The notion of writing for audience had been well-developed in some schools, and emphasis placed too on developing pedagogic approaches, which stressed pace, variety, structure and immediate plenary feedback, which were thought to favour boys. Yet other approaches stressed the need to engage boys more actively in the school as learning community, to combat notions of laddishness in young boys through the creation of 'Executives' and 'Buddies' to give responsibility and support in the playground and the classroom, and to generate a sense of enhanced self-esteem and self-worth (Warrington et $a l, 2003)$. In the secondary sector, merit systems had been introduced, for example, in an effort to improve motivation, and short-term target-setting strategies were implemented; students had been offered mentors (in some cases other pupils, or staff, governors or members of the local community) with whom they could talk about their progress; other initiatives focused on specific departments, analysing teaching materials, resources and assessment strategies, examining the pattern of classroom interactions and the focus, direction and purpose of teacher-pupil talk (Younger et al, 2002).

Within this range of initiatives, we encountered a number of distinct challenges, however:

- Most crucially, even in schools which had been taking the issues seriously for several years and, in the process, were undertaking a number of interesting innovations, there was little real evidence about whether some interventions were more successful than others. Most innovations had not been subjected to anything approaching rigorous evaluation, and there was little grasp as to how and why ideas which appeared to have worked in one school or setting had worked and been effective.

- The schools we initially worked with did not always know, therefore, why their gender gap had closed. Certainly all schools had put into effect certain measures to raise improvement, but these were not necessarily gender specific, nor indeed boy specific. Thus, although there was often an intuitive grasp of why the gap was narrowing, there was little analysis of the process of impact which would enable the strategy to be transferred, with confidence, to other schools.

- To complicate things further, no school had focused on just one form of intervention. We found that schools tend to employ a basket of strategies, particularly primary schools. Sometimes the basket contained explicit, clearly articulated strategies, but it often contained approaches that had become such an implicit part of the culture of a particular school, that the school found it difficult to acknowledge that it was doing anything special. It was, therefore, a considerable challenge to disentangle the process of change within the school, and to evaluate the specific effect of particular interventions. 
At one level, then, our pilot study supported previous work (Arnot et al, 1998) in confirming that in many instances, approaches and strategies had often been tried on an essentially ad hoc basis and, for reasons which are perhaps understandable given the conflicting pressures on schools, not been properly evaluated and their potential identified. Crucially, we became aware that, even where these schools appeared to have in place intervention strategies which were working effectively to raise boys' (and girls') achievements, little attention had been placed on defining how these ideas might be transferred to other settings within the school and indeed, sustained through time in the face of staff loss.

At another level, however, a number of interesting, if diffuse, strategies were identified within the pilot study which did seem to have some potential to help transform the achievement of boys (and girls) in specific contexts. Some of these strategies were shortterm and tightly focused, but the more promising seemed to be developing within a more holistic framework. Here schools acknowledged the complexity of the issue of students' under-achievement and recognised the plurality of 'boys' and 'girls', rather than making homogenous and stereotypical assumptions about how 'boys' and 'girls' learn and might be engaged with schooling. This challenging of assumptions about the nature of 'boy' and 'girl' intensified the challenge for the project team, but we hope it gives our work more validity and credibility, and will enable teachers and schools to recognise the reality of the situation they are operating within.

These intervention strategies have evolved, therefore, as we have engaged with headteachers and with teachers to address a number of fundamental issues which they have identified as crucial in affecting boys' and girls' learning. We have classified these intervention strategies into four broad groups - pedagogic, individual, organisational and socio-cultural - according to their predominant emphasis and characteristics, although inevitably no one group is exclusive of the others:

- Pedagogic strategies have included approaches which are classroom focused, relating to teaching approaches, analysing classroom interactions and dynamics, exploring the role of collaborative and competitive activities within the classroom, and exploring issues associated with accelerated learning and preferred learning styles.

- Individual strategies have usually focused on some form of mentoring and targetsetting, aiming to enhance students' confidence and self-assurance as learners, and to monitor individuals' progress through tutorial systems and approaches which focus on the academic as well as the pastoral.

- Organisational strategies emphasise whole school approaches, where schools attempt to develop a school ethos and culture in which achievement in all sorts of areas is an expectation which is celebrated, and accepted as the norm. Such approaches include developing a leadership (or prefects') system, examining and utilising data, implementing single-sex groupings on a shorter or longer time scale, sometimes in particular subjects, organising lunchtime and twilight support clubs, and developing high profile praise and reward systems.

- Socio-cultural approaches: those which attempt to change images of laddish masculinity held by the peer group or perhaps the family and community, and to develop an ethos which helps to eradicate the 'it's not cool to learn' attitude 
amongst boys. These often involve the early identification and de-fusing of antiwork groups, helping students to use friendships positively and as a support for learning, and developing key befriender / key leader schemes.

\section{Research Design for the Intervention Stage}

\section{Selection of schools}

The research design which structured the work in the intervention stage within the Raising Boys' Achievement project (2001-2004) involved the team in the identification of a number of key schools. The same criteria used to select schools in the pilot phase of the project (see page 27) were applied, using national data sets supplied by the DfES. Thus in the intervention stage of the Project, primary schools were identified where the gap in average points score between girls and boys in English at key stage 2 had declined through time, in response to well-defined strategies. Table 3:1 shows one example of a two-form entry school where both boys' and girls' Key Stage 2 results in English had improved over the period 1996-2000, but where boys' results had improved at a much faster rate than average, and to an extent where the gender gap was in favour of boys.

Table 3.1: Comparison of boys' and girls' mean points score in English, 19962000

Data from an Inner London 3 - 11 school

\begin{tabular}{|c|c|c|c|c|c|c|}
\hline & 1996 & 1998 & 2000 & $\begin{array}{c}1996-2000 \\
\text { school } \\
\text { improvement } \\
\text { rate (\%) }\end{array}$ & $\begin{array}{c}\text { National } \\
\text { comparison } \\
2000\end{array}$ & $\begin{array}{c}1996-2000 \\
\text { national } \\
\text { improvement } \\
\text { rate (\%) }\end{array}$ \\
\hline Boys & 19.56 & 21.91 & 27.00 & $38.0 \%$ & 25.70 & $10.1 \%$ \\
\hline Girls & 24.79 & 24.53 & 25.00 & $0.8 \%$ & 27.40 & $7.7 \%$ \\
\hline Ratio b /g & 0.79 & 0.89 & 1.08 & & 0.94 & \\
\hline
\end{tabular}

Similarly, secondary schools were identified where differences between the average points score of boys and girls in their GCSE examinations had narrowed over the period 19962000, again within the context of a rising trajectory of results. Thus, in both phases, schools were selected where this narrowing of the trend lines between girls' and boys' performances reflected boys' performances improving at a greater rate than girls, but in both cases trends through time indicated improvements over most of the time period studied (1996-2000). We acknowledged that, given schools' individual circumstances and relatively small sample size, the average points score of girls or boys might dip in one particular year, compared to the previous year, and we accepted a one-year tolerance within the year-on-year sustained pattern of improvement. Thus, in the example used in Figure 3.1, although the levels of academic achievements of both boys and girls were lower in 1999 than in 1998 (as measured by the average points score achieved), the trajectory of achievement of both boys' and girls' achievements at GCSE increased 
through time, and the gender gap narrowed from 7.5 percentage points (1996) to 5 percentage points (2000).

Equally, schools were only selected for further involvement in the project when senior staff were able to articulate why this 'narrowing of the gap' was happening, and to identify the strategies which had been explicitly developed to raise boys' achievements without detracting from those of the girls within the school.

Fig. 3.1: Average GCSE Results by Gender, 1996-2000

Data from an East Anglia 11-16 school

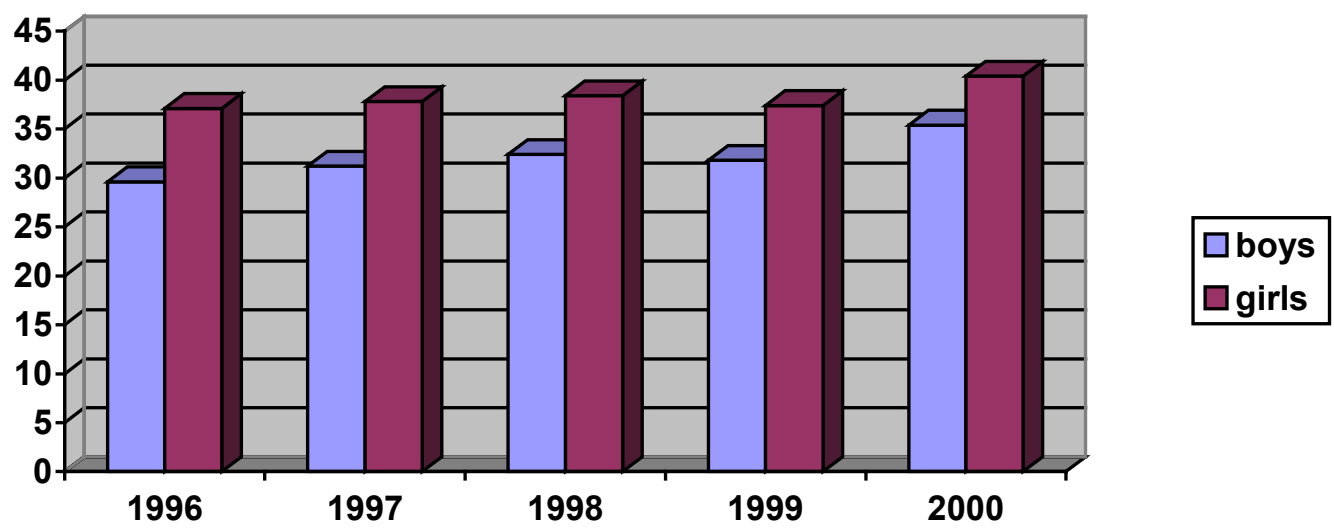

This process of identifying possible key schools was not easy to implement (Younger et al, 2002). In fact, very few schools across the whole of England met these criteria - fewer than $0.5 \%$ of all schools - suggesting that it is very difficult to sustain the closing of the gap over even a few years, and as already pointed out, the initial criteria had to be relaxed slightly in order to find enough schools to work with. A further difficulty, as telephone interviews with senior staff sometimes revealed, was that schools were not always clear why or how the school was having this impact on boys' achievements. In primary schools in particular, a 'basket' of strategies was often in place, and sometimes it was difficult to identify a precise approach.

Despite these difficulties the project team was able to select eight primary and eight secondary schools to begin work in the academic year 2000-01. We called these 'Originator' schools, since each had in place a strategy which had contributed towards the transformation of the achievements of boys (and girls) in the preceding four years. Our next task was to select two 'Partner' schools to work with each Originator school as a 'triad' (group of three) schools. The Partner schools were similar in terms of their socioeconomic context, student numbers and age-range to their linked Originator school. In the primary sector they were located within the same local education authority area and often in close proximity; in the secondary sector, however, because of issues of competition between nearby schools, we chose triad schools some distance apart, usually in neighbouring authorities. Key Stage 2 and Key Stage 4 data for the Partner schools revealed a gender pattern of results similar to the national average: in other words these were schools which had a consistent gender gap in favour of girls. 
The particular nature of Special Schools, and the fact that there are far fewer of these types of schools, meant that different selection criteria were used. These are discussed in Chapter 10, which is devoted to special schools.

\section{The research strategy: aim}

The aim of the research strategy was for school colleagues in each Originator school, working with a member of the research team, to define the core of the intervention strategy which had led to rising levels of achievement among the boys in that school, and to attempt to transfer it, with contextual specific modifications, into their linked Partner schools. As time passed, it was envisaged that this process would support clarification of the strategy within the Originator school, and help to sharpen the focus and effectiveness of the strategy - and to further improve achievement levels - in the Originator school. At the same time, it was hoped that, as the intervention strategy became embedded in the Partner schools, boys' achievement levels there would rise.

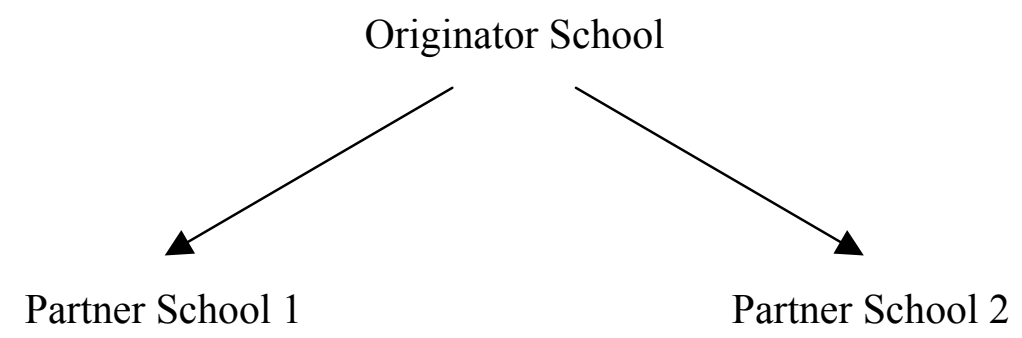

In some triads, this research design worked as we had anticipated, with the Originator school acknowledged as such within the triad because of its expertise and its performance profile, and with this school initiating and sustaining the lead throughout the four years of the project. In other triads, however, a more collaborative structure emerged (see Chapter 11), with a Partner school assuming leadership as the triad developed, refining and elaborating the initial ideas from the Originator school and driving these forward in a new direction.

\section{The research strategy: process}

As mentioned above, each triad was assigned a member of the research team as link researcher. One of the main authors of this Report undertook overall responsibility for the secondary and special schools working with the project; the other oversaw the primary aspects, with both also taking responsibility for individual triads. A full-time research associate took responsibility for some triads at both secondary and primary level, a colleague with expertise in literacy worked with two primary triads focusing on literacy, and another colleague with expertise in special education led that aspect of the Project. The Project also employed seconded teachers as link researchers in some of the triads. The qualitative aspects of the research were underpinned by a feminist methodology (McDowell, 1992, Moss, 2002, Roberts, 1981) which tried to take into account the positionality of the various members of the research team. Thus we are fully conscious that a shared ethnicity, together with different genders, ages, areas of expertise and levels of experience among the interviewers, will have had some impact on the qualitative data 
we collected from various students in the course of the Project. While such impacts are inevitable, particularly in such a large project, regular meetings of the research team ensured that, as far as possible, a common approach was adopted, with full discussion of the research instruments used at different stages.

In terms of the actual research process, both quantitative and qualitative methodologies were used in order to address this issue of 'under-achievement'. Analysis of DfES databases helped not only to identify Originator and Partner schools, but also allowed access to a consistent database through which to track trajectories in average points scores among the schools we worked with. We have also drawn upon the various developments in the value-added debate, to try to identify progression in achievement across key stages 1 to 4. Teacher assessments and records from non-statutory tests within the schools themselves added to the quantitative picture we were able to develop for each school.

At the beginning of the intervention stage, link researchers from the Project undertook an intensive interview with the Headteacher, or other link person in each of the schools, in order to understand each school's approach to gender issues and to discuss any specific strategies which had been implemented in order to address disparities. Documents such as prospectuses, relevant policies and Ofsted reports were also collected at this stage to provide a fuller understanding of each school's context.

In every triad school the link researcher undertook a common 'before' and 'after' methodology. Four common interview guides were developed: two were used at the beginning of the research process, one for all primary schools and one for all secondary schools; the other was used at the end of the two years of the intervention strategy. Schools were asked to select two or three friendship groups of boys of a range of abilities who were thought to be achieving at a lower level than might be expected, drawn from year 5 in primary schools and year 10 in secondary schools. Parental / carers' permission to interview the students was sought through the schools; the purpose of the interviews was also explained to each group of students, who were asked if they agreed to being interviewed. The first of the focus group interviews aimed to explore students' views on any perceived differences between boys and girls in their school, their views of school and their own achievements, how they perceived levels of support for their school work and what might help them to improve. Final focus group interviews, insofar as possible with the same groups of boys, used some of the same questions again to explore any changes in attitudes, and well as including questions to identify any perceptions of change as a result of specific strategies.

During the main stage of the project the link researcher worked intensively with schools, liaising through telephone calls and email, and spending two days each term in each school. In addition, a termly triad meeting gave key staff the opportunity to come together with the link researcher to share experiences, to explore issues and to clarify understanding. Initially the triad meetings were used to define the essence of the intervention strategy to be adopted, the particular year group to focus on, and the process factors seen to be important for the diffusion and embedding of the strategy in the Partner schools. Staff also occasionally visited other schools in their triad to see the interventions in practice and to offer critical and reflective support. The triad meetings and associated school visits by the research team have been at the heart of the project: they have enabled the intervention strategies to evolve, to be evaluated in practice, and to be sustained. This 
pattern of regular meetings and visits have offered support where the attempted transfer was meeting resistance, they have allowed strategies to evolve in different directions (in Originator schools as well as in Partner schools), they have created a sense of togetherness and common purpose, and they have allowed the authenticity of the intervention to be verified - and on occasions, challenged.

Another central aspect of the research process was annual conferences held separately for primary and secondary triads. Special school representatives joined conferences in the relevant phase, and also met together as a group on three occasions. Other schools were brought together from time to time for specific purposes: for example, a day conference was held for primary triads in the London area; another day conference was held for primary and secondary triads working on teaching and learning styles. The purpose of these various conferences was threefold: to build a common purpose and identification with the ethos of the Project, to disseminate findings and share good practice both from within the Project and more widely, and to enable cross-triad links to develop, particularly across triads working on the same kind of strategy.

Various methods were employed during the pilot phase, main intervention stage and extension phase of the Project:

- Focus group interviews with groups of boys (and girls)

- Individual interviews with boys (and girls)

- Interviews with teachers

- Visual methods (inviting primary age pupils to respond to photographs)

- Classroom observations

- Questionnaires to students, staff and parents

- Attitude surveys

- Analysis of pupils' writing through time

(All interviews were structured or semi-structured - see Appendix for examples.)

Because different kinds of strategies were used in different triads, and because the research ran across primary, secondary and special schools, not all methods were appropriate to use in all contexts, and the precise mix of approaches was determined by the context of the triad and the specific strategy being implemented. In all schools, however, listening to pupil voice was a central part of our approach (Rudduck et al, 1996, Rudduck and Flutter, 2004), and thus interviews with students were a central aspect of data collection in every school. These enabled students' perspectives to be heard on how the strategies impacted upon their learning and on their attitudes towards the learning process through time.

In all the primary triads and in some of the secondary triads the impact of the strategy was assessed through a focus in each school on a group of 8-12 'under-achieving' boys, who were interviewed on several occasions throughout the project. Detailed case studies were built up for each boy, including attainment through time; where appropriate writing samples were analysed, and classroom observations focused on these pupils. In several of the triads, particularly at secondary level, questionnaires were used to gain a wider response rate to whole class interventions. The precise mix of methods employed in each triad is discussed in more detail in the following chapters, which present case study material from across the range of strategies used in the RBA project. 


\section{CHAPTER 4: Pedagogy I: Raising Achievement in Literacy at Key Stage 2}

Boys' lack of engagement with literacy has been identified as one of the most significant factors in accounting for their lower attainment in relation to girls (Ofsted, 1993a; Barrs and Pidgeon, 1993 and 2001). Writing, in particular, is highlighted as an area where boys show lack of motivation. However, as the 1998 Ofsted report into gender and school performance noted, blanket statements about boys' and girls' attitudes or attainment do not help in unpicking the complexities of under-achievement in certain curriculum areas (Arnot et al, 1998). Research carried out by Moss (2000) takes a rather more carefully defined look at boys' reading, suggesting the need to acknowledge that some boys do not present problems in their approaches to literacy, and a recent Ofsted report (2003a) focuses on schools where boys perform well in writing. More recently, too, there have been challenges to negative views of boys and literacy (Safford, O'Sullivan and Barrs, 2004) with fears that stereotyping may lead to restricted policy. Nevertheless, whilst there have been shifts in the kinds of approaches taken by researchers and policy makers over the last ten years, the gap in national scores for boys' and girls' literacy remains (Chapter 2).

Boys' under-achievement in literacy has been described and analysed in terms of different perspectives: personal factors, such as motivation, or lack of it (Ofsted 1993 and 2003a); the lack of strong male literacy models (Barrs and Pidgeon, 1993); teachers' perceptions of behaviour (Myhill 2000); teaching approaches (Frater, 2000; PNS/UKLA, 2004); the content of the literacy curriculum (Marsh and Millard, 2000) and class and ethnicity (Gillborn and Mirza, 2000). The complexity of the area is matched by the range of different types of evidence which inform debate: some is based on national testing data; some on teacher/practitioner research; some on observational data. Alongside this variation, literacy itself is a shifting concept in the different research perspectives and evidence of achievement in literacy can be differently conceived. The schools in the Raising Boys'Achievement project which took literacy as a focus shared some common ground: they saw successful literacy as measured by test results and processes, as evidenced by pupils' growing ability to operate as literate individuals.

\section{Approaches to Literacy: processes and products}

Any research into promoting standards of literacy has to confront the complex relationship between the products of reading and writing and the processes of learning to become literate. Taking a focus on boys' literacy adds to these complexities. In order to provide evidence of how standards of achievement and attainment might be secured, the research and intervention plans of the triad schools had to take the dual perspective of "teaching reading' and 'teaching writing' as well as a view of what is involved in 'becoming a reader' and 'becoming a writer'. The schools have provided several kinds of evidence: performance in National Curriculum tests, mainly at key stage 2 and trends within the schools involved; teacher assessments; pupil perceptions; classroom observations; analyses of writing and evaluative data from teachers and pupils. Over the time schools have been working on the project, it has become clear that local, even school-specific, situations vary greatly and have their effect on achievement, and that issues about raising standards of literacy cannot be separated from wider socio-cultural issues. The diversity of the settings and circumstances of the schools involved in the project is matched by the diversity of the 
findings. Nevertheless, some common threads have emerged which suggest the importance of taking a longer-term view of how schools might best raise standards of boys' achievements in literacy.

Three primary school triads focused on literacy. Early involvement indicated that the emphasis in each triad would be slightly different to accommodate the context of each school and area. One inner city triad of schools, where the population is culturally and ethnically very mixed, identified reading as more of a challenge for boys. In the two other triads, one in a largely white, suburban setting and the other in a south coast holiday resort and associated suburban areas, writing was the main focus, although each took different routes to raising boys' achievement. All the triads included key stage 1 pupils at some stage of their work, although this is not reported here as there was not sufficient work to offer generalised conclusions. The junior and primary schools focused on years 5 and 6 . Within each triad, whether the focus was on reading or writing, there were specific issues that the schools wished to address. Each of the triads worked on an initial phase of intervention followed by a shorter second, extension phase. The three case studies which follow, although representing particular and specific features, highlight some of the key classroom and whole school issues which need to be considered when undertaking any initiative to raise boys' standards of literacy.

\section{Case study 1}

\section{The background}

The schools in this triad were situated in an inner London borough. The Originator school was a large primary school of over 400 pupils, located in a ward which falls in the bottom $3 \%$ of wards in terms of multiple deprivation according to the 2000 Index of Deprivation. The infant school in the triad, again very large, with a population of 300 pupils, was also in the bottom 3\% whilst the third, a junior school of 360, was in the bottom $7 \%$. A very high proportion of the pupils were bilingual or multilingual. Performance at key stage 2 in both schools with older pupils was well above the national average for similar schools in 2002. National curriculum test results for four years before the project began showed a slight gender gap in favour of the boys in literacy in both schools. There was no comparable information for the infant school, but the impressionistic picture was that many of the boys were under-performing, often because of pupil turnover and disrupted experiences of schooling.

\section{Reading - involving homes and families}

The emphasis in all schools was on reading and involvement of homes and families with the aim to raise boys' self esteem and achievement in literacy and to continue and strengthen links with parents. All the schools investigated parents' and carers' attitudes to reading and each selected focus groups of boys who would be tracked during the project. The response to surveys of parents' and carers' attitudes to reading yielded some unexpected outcomes. In the infants school, adult male family members indicated less confidence about literacy than female family members. This was a surprise since it is often assumed that female family members from the ethnic groups represented in the school would have shorter educational experience than the males and therefore limited access to learning. At key stage 2, the surprise was that many of the boys held very negative views 
about the reading practices of school whereas adult male carers responded with enthusiasm about their own (considerable) reading experience and choices at home.

After these initial surveys, it became clear that the priority should be to raise the profile of reading as a pleasurable activity in school rather than focus on reading practices at home. The aim of including more adults was not lost, but the emphasis shifted to including them in school-based reading where possible. There was also a sense that it was important to develop positive views of reading amongst the fathers of the future - the boys in the classrooms at the time. Each school selected focus groups of boys who were perceived as underachieving in reading, or in literacy more generally. They were interviewed at various stages during the project and their reading scores kept throughout. The boys' responses in interview and the initial perceptions surveys strongly suggested the significance of the social aspects of learning, where friendship groups and family members were seen as very important both as support and example.

The junior school (Partner school A) established a range of approaches to reading based on more extensive discussion of texts and less of a 'testing' approach. This included explicit attention to teachers modelling ways of responding to the meaning and content of books, not just decoding the text, and using reading journals on a regular but not routine basis as a reflective space to record, by choice, response to texts. The Originator school began setting homework for years 5 and 6 which specifically encouraged pupils to read all kinds of texts and set up a pilot 'reading buddy' scheme between unmotivated year 6 boys and underachieving year 3 boys which, in the second phase of the project, was extended to other schools in the borough.

These approaches raise immediate questions about any ideas of 'transferable strategies' to raise standards of unmotivated boy readers. What these schools identified was not so much a gap in teaching reading but a need to focus on how to encourage boys to become successful and satisfied readers. It was recognised that all the relevant reading skills had to be in place, and, indeed, the reading buddies were given explicit instruction in the range of strategies involved in teaching reading to the younger boys. But for many of the pupils, even the youngest, it was not so much a matter of knowing how to decode text on the page but of having a sense of 'what's in it for me'. Talk and reflection about reading, sharing ideas about what was enjoyable to read, between adults and pupils and between the pupils themselves, were the means by which the technical skills of reading were successfully linked to the boys' development of greater discrimination and independence as readers and, eventually, higher standards of reading for most of them.

It became clear that discussion of reading made an important contribution to raising standards of reading. The key question, however, is how opportunities for conversations about reading can be managed and how the social aspects, which were obviously so important to the promotion of engaged reading, can be provided for in classroom teaching of reading. There is a fine balance to be held between relaxed talk about reading which could possibly become desultory, even though pleasurable, and planned and purposeful exchanges between partners about reading which will genuinely promote interest and engagement. In this triad, the schools were clear that discussing what you were reading as part of learning how to read must be an essential element in a planned and systematic approach to teaching reading. 


\section{Assessing progress in reading at key stage 2}

At key stage 2, both numerical and interview data, as well as teachers' informal observations, indicate the success of the approaches used. In both schools in the original triad with year 6 pupils, there were marked gains in teacher assessed reading scores in the focus groups of boys. The expected rate of progress is usually judged as one National Curriculum level over two years, often recorded in thirds of levels as part of regular record keeping in schools. The teacher assessments indicated that from the two focus groups (29 boys in all) there were remarkable gains (see Table 4.1) with over half the group far exceeding expected yearly progress.

Table 4.1: Teacher assessments of reading progress in the Year 6 focus group boys in National Curriculum Levels (no. of pupils)

\begin{tabular}{|c|c|c|c|c|c|}
\hline $1 / 3^{\text {rd }}$ Level & $2 / 3^{\text {rd }}$ Level & 1 Level & Between 1 and 2 levels & 2 Levels & More than 2 Levels \\
\hline 2 & 11 & 9 & 3 & 2 & 2 \\
\hline
\end{tabular}

In interview, the focus group boys commented on their own progress towards greater independence and responsibility for their learning. When asked specifically about reading many were able to articulate exactly the kind of progress they felt they had made. Some were about technical skills and decoding strategies:

I've learned strategies to help me read faster.

I read the whole sentence.

Break it up.

Some revisited the early views about the importance of others in making progress with reading:

My brother's helped me pronounce the words... I read more now.

I think I've improved in reading. Teachers have helped me... I'm more fluent now. I know I've been prepared for secondary school.

Many commented on their preferences in reading:

Reading is fun. I like reading long books and comics ... and books from films.

I'm reading more often - picture books and horrible histories, autobiographies about famous people (Bruce Lee and Neil Armstrong).

In evaluating the reading buddies project, the boys were able to comment even more specifically about how to promote successful reading. This project had been very effective, both socially and in terms of reading progress. The younger boys' reading improved and the older boys showed high motivation to work as mentors:

Before, I didn't understand PSHE - things that our teachers told us - but after the buddy reading scheme... at first I thought it was teaching but now I know it's about friendship too.

In interview, and during observations of the reading buddies in action, the year 6 boys commented on reading strategies, using vocabulary which might be more associated with 
teacher language: initial sounds, picture clues, letter clusters, meaning, comparisons with other words, for example:

Shane is more fluent now and used picture clues more. I might try to make it fun by putting it in pictures... and making it more physical to help him learn.

They also noted the improved attitudes of the younger boys and the effects on reading behaviour:

If somebody doesn't like reading then they read more and more... and they like to read.

I think reading buddies are good because there are more children in the community who will learn to read and write... and when you grow up you might hear from them, saying 'Thank you for helping me in reading, and now I've got a great job because of your help'.

Interview and observational data add to the evidence about the importance of talking about reading: the reading buddies project, particularly, indicated the value of giving pupils the opportunity to develop a language to talk about reading - a meta-language. The year 6 focus group boys confidently used technical terminology about reading strategies and about the range of texts which were on offer to them, thus extending and enhancing their capacity to make reasoned and thoughtful judgements about the processes of reading and the kinds of texts they enjoyed reading.

\section{Extending the strategy}

In the final year of the Project, the reading buddies programme (re-named 'Boys Supporting Boys') was disseminated to three further schools in the same local authority. Responsibility for coordinating this stage was taken by the two members of staff who had implemented and developed the programme in the Originator school. The three additional schools served similar catchments, with pupils from a diversity of backgrounds, high pupil turnovers and very high proportions of pupils with free school meals eligibility, special needs and English as an additional language. An initial meeting was held with the coordinators in the new schools to explain the strategy, and resource materials were provided. In each school a group of under-achieving year 6 pupils (mainly boys) was identified and trained, and the programme ran in all four schools over the course of the project's final year, with a project researcher interviewing groups of boys before and after the strategy. The year 6 participants completed a simple questionnaire, quantitative data were collected, and an end-of-year meeting took place to review progress.

Sustaining and refining the strategy in the Originator school led to its continuing success in terms of significantly improved results in reading: Table 4.2 shows a comparison of the reading levels of the focus group boys at the beginning and end of year 6 in terms of national curriculum levels. Improvements were also seen in concentration, behaviour and attendance, and in the boys becoming more responsible and thoughtful. Questionnaire results from this school showed very positive responses: all the year 6 boys thought their buddies' reading had improved, and all felt they had gained from the experience:

I think reading buddies was excellent because it helped young people learn to improve their reading plus I have learnt a lot from the two buddies I've had. 
Table 4.2: Progress in Reading Levels of Year 6 Focus Group Boys in Originator School (no. of pupils)

\begin{tabular}{|c|c|c|c|c|}
\hline Less than $1 / 3^{\text {rd }}$ Level & $1 / 3^{\text {rd }}$ Level & $2 / 3^{\text {rd }}$ Level & 1 Level & Between 1 and 2 levels \\
\hline 1 & 4 & 5 & 3 & 1 \\
\hline
\end{tabular}

In two of the other schools outcomes were also positive. Tables produced by the DfES (2003) allow a 'value-added' score to be calculated to measure progress for each child based on performance in core subjects from Key Stage 1 to Key Stage 2. The value added measure for the group of 11 boys between Key Stages 1 and 2 in one of the schools was 102.4, compared with that of the school as a whole of 100.4, indicating that the Boys Supporting Boys programme may have had a particular impact on these boys. All but one of the boys in this group said they had enjoyed reading club, and most felt it had improved their own reading as well as that of the year 3 pupils. Attitudes to reading changed, too:

At the beginning when we started talking about what kind of books we liked, my partner used to just bring books what I didn't like, but then I started liking it because I used to read it, and he used to read it as well, so I quite like the stories and how they used the words. So now I really like different kinds of books as well.

In both of these schools, the fact that, although several of the boys said they did not like school but enjoyed reading club, may be an indicator of its success.

In the third school there were also some successful outcomes, with 7 of the 12 boys involved improving their reading grades by more than one National Curriculum level during year 6 . The boys themselves were much less positive, however, with most not enjoying reading club and feeling it had been imposed on them against their will, although two thirds of them did feel that they had made progress with their reading as a result. One of the difficulties of the scheme in this school was it had been run by a teaching assistant who appeared to have problems in managing discipline problems which had emerged, and who consistently lacked support in this role.

The gains from the reading buddies programme, both in the intervention stage of the project and in the final year, were very evident, both in their effects on attitudes and selfesteem among the chosen boys, and in their impact on reading progress. Research across all the schools involved showed, however, that there were practical problems which need to be addressed if such an initiative is to succeed. In particular, if year 6 boys who already show some reluctance to engage in school-based literacy are asked to give up their lunchtime leisure time to participate in a reading club, this has to be sensitively handled, with support at whole school level in terms of organisation and strong reinforcement given to the boys for their participation. Once this issue of timing is resolved, and with training for the year 6 boys in place, together with a range of accessible reading material and careful teacher monitoring and coordination, our research has shown this strategy to be particularly successful in terms of transfer to other schools. It is expected that three further schools will join a self-sustaining continuation of the project in the next academic year. 


\section{Case Study 2}

\section{The background}

This triad, situated in an outer London borough, focused on writing. The schools, according to the 2000 Index of Multiple Deprivation, were within the $12 \%$ least deprived wards in England. The Originator school consistently maintained high key stage 2 SAT results. Since this large junior school (359 pupils) shared a site with the infant school (268 pupils), which was also successful in maintaining high SAT results, both schools participated in the project. The third school, a one form entry primary school of 201 pupils (Partner school B), also with high general standards of literacy, allowed useful comparisons to be made in terms of analysing successful school practice as well as looking at how, even in high performing schools, boys' achievements in writing might be further promoted.

At the start of the project there was a very small gap in favour of girls' performance in English at key stage 2 in both schools. However, in the Originator school boys' results in English had experienced a general upward trend over five years. In Partner school B the results had been more erratic, although boys had consistently outperformed girls in national curriculum science and maths tests in five of the previous seven years. Gender had not been apparent as a 'problem' area for either school at key stage 2 . In the triad as a whole, most pupils were of white UK heritage and the percentage of pupils identified as having special educational needs was below the national average.

\section{A whole school approach}

Since all three schools had high national test results in English, particularly for boys in relation to the national average, a first stage before developing strategies to improve writing was a rigorous examination of school and classroom factors which may have contributed to this success. Despite much recent work on school leadership and school improvement (MacBeath and Moos 2004; Hopkins, 2001) it is not always easy to identify factors which contribute to a school's success. Whilst the schools in this triad were sited in relatively prosperous areas, the pupils were by no means all from affluent homes where high achievement might be expected. Partner school B necessarily took pupils from all backgrounds and pupils with difficulties were often referred to the infant and junior schools because of their good record. The headteachers' views and experiences were similar: all had been heads for 10 years or more; their initial concerns on appointment had been to raise the profile of the school but then all reported shifting the focus towards greater coherence in teaching and learning. Interviews with parents from the three schools indicated that good relationships had been established and maintained.

An extensive review showed several factors which seemed to be significant contributors to high standards, and among the most important were those which form the pre-conditions for the successful implementation of any strategy, and which are brought together in Chapter 11 (see p. 132). In addition, the coherent and integrated planning and inclusion of ICT and creative aspects of the curriculum were high profile, combined with opportunities for pupils' personal development beyond the scope of lessons. There was an emphasis on partnership, with positive relationships with parents and carers and strong support for, and involvement in, school activities, as well as effective teamwork between governors, staff 
and parents in determining school priorities. The schools aimed, too, for continuity of experience, not only for the pupil but for teachers, so that links were made between different aspects of the pupil's learning experiences and the teaching.

\section{Becoming a writer}

The self scrutiny undertaken by the schools allowed them to focus more precisely on areas of writing which might be improved. In early interviews, the boys who were identified as underachieving in writing presented views which were very much in line with other research into pupils' perceptions (UKLA/PNS 2004). Many of the boys disliked writing, with the exception of writing stories, and their view of writing was mostly focused on technicalities of spelling and handwriting. Analysis of their writing samples showed a lack of personal voice; a lack of awareness that the piece was meant to communicate with a reader; and insecure structure in terms of paragraphs and punctuation. There was also a tendency to write from inner visual images and a failure to translate these into coherent written form.

As a result of these analyses, the overall triad strategy concentrated on moving from 'learning to write' (technical skills) to 'becoming a writer' (understanding the meaning and other dimensions of writing). There was already strong emphasis on speaking and listening, particularly in the Originator school and this increasingly became a focus of attention in the triad. The aim was to develop an integrated approach to literacy in its broadest sense, seeing reading, writing, speaking and listening not as separate components, but as interrelated.

Teaching approaches which would be developed during the project put emphasis on:

- greater attention to paired and group talk and drama; more oral preparation for narrative;

- work on the elements of narrative; more explicit discussion of character, plot, setting, structure and vocabulary;

- teacher demonstration: reading, discussing and analysing ideas, 'thinking aloud';

- aligning each curriculum area with a specific text type as designated by the Literacy Framework for specific terms and year groups;

- the visual as a source of inspiration for writing and use of visual approaches to planning and note-making.

By the end of the project, all of the focus group boys' interviews referred to satisfaction about writing and they were much more able to talk precisely about how to write. Their comments reveal genuine shifts in understanding. Overall, they became much more assured with the language of writing:

I like the way I've used the past tense.

I've started to use more adjectives.

I've got better at setting a scene and explaining what I think.

When asked what advice they would give younger writers, planning strategies and visual aspects of the imagination were important: 
Use bullet points in non-fiction writing because they make sure you say the facts. STICK TO THE PLAN!!

Plan out before writing a story. Try and pick out the key events. Describe the setting and feeling well. See and picture it.

Write down your ideas in your plan. Eliminate bad ones and write the best ones. Try to create a picture in your head.

Several of them were explicit about how they took ideas from personal experience including listening, reading, and watching film and television:

You get the voice in your brain.

I read a lot... I shut out everyone around me ... I like characters in my stories, I make a mixture of all the people I know....

When I watch movies I see the story so when I write I see the action.

They were able to talk about the requirements of different text types and about the decisions they had to make when writing. Overall, from their earlier concerns about the surface features of writing ('learning to write') they had moved towards a clear sense of what 'being a writer' involves in terms of both technical and imaginative elements:

Make long sentences but don't make them boring or repetitive and use lots of punctuation. Work with someone you can work with.

You should stop and think of what you're going to do and see it in your head.

Use your imaginations and see what actually happens in your head. Write something you want to write.

Analysis of their writing showed marked improvements in the pace and structure of story writing, as well as identifiable individual preferences and strengths: for character depiction; evoking atmosphere; drawing on visual memory of narratives; using dialogue as a narrative device. In non-fiction writing there was an equally marked awareness of the needs of a reader: the extent and clarity of information required to communicate effectively. These developments in writing align with the specific approaches to teaching writing adopted by the teachers.

\section{The importance of talk}

Building on their preliminary work about boys' writing, the Originator and Partner school $\mathrm{B}$ extended their focus in phase two to give more explicit and attention to speaking and listening as a means of supporting writing. They also aimed to extend the work beyond years 5 and 6. The Primary National Strategy introduced guidance for speaking and listening in summer 2003. In the junior school, this prompted a thorough review of planning specifically to incorporate forms of speaking and listening which would directly support the teaching of particular text types.

Teachers in both schools were positive about the effects on themselves and the pupils:

I've improved the tasks and can see the clear objectives of what we want to do. I can see the positive aspects of it.

Because I've seen the improvement it's encouraged me to do more. I feel more confident. You can experiment a bit more. 
It's added a new dimension because it fits with the Strategy's speaking and listening focus... this integrated approach of speaking and listening as an automatic part of literacy work is now part of my normal planning and teaching.

Pupil interviews showed that even the younger boys had developed a sense of how partnered talk helped with their writing:

When you're talking it makes you have more ideas and if you have a partner it makes you feel more confident.

When you're working... when you're writing it's like you're learning as well at the same time. You always have to talk to yourself and your friend about something.

If you don't really know what you're doing, you should talk.

By Year 6, there was even more conviction about how talk contributes to learning and writing:

It does a lot... if you explain to others you understand it.

Yes it can, when you're talking to yourself... telling yourself how to do it.

Yes. You make your story up in your mind and plan it in your head ... If I speak to David or Chris about it .... I've got all the bits and I don't know where to put them... then it helps me - and them

The security of sharing ideas before writing had helped the pupils to be more adventurous with their writing, taking greater risks both in the language they were using and the structure of their work as a whole. There was also evidence of greater enjoyment and a sense of I can do it. Teacher assessments showed that the boys' writing had improved more than expected, specifically in the development of pace and individual voice in their writing; the establishment of atmosphere; a clearer structure and improved punctuation and paragraphing.

\section{Classroom approaches to support boys' writing}

The work of this second triad serves to emphasise the picture of complexity outlined in Case Study 1. In particular, it stresses whole school policy and planning and the impossibility of separating writing from language teaching as a whole. In this triad the emphasis was on cutting into a vicious cycle of boys' negative perceptions of themselves as writers and learners. Using 'boys' as a descriptive category for a group of learners can, however, tend to ignore differences between boys. Over-generalisations are not helpful and several of the boys involved in this triad's work showed that they were all too aware of public views and were affected by media representations of them. The sensitivity to diversity shown by the schools in this triad allowed analysis of factors which can support successful writing for boys (and girls):

- the organisation of literacy sessions to incorporate speaking and listening and IT;

- an emphasis on talk and time to reflect - finding ways to talk about learning and literacy;

- the importance of 'companionable' writing through using response partners and group work;

- a move away from commercial schemes for teaching writing towards more innovative literacy sessions; 
- teachers being prepared to take risks in bringing more creativity to literacy sessions;

- covering a range of writing types but also teaching (and allowing choice of) different ways to approach writing;

- no unnecessary writing: writing only required where it is central to learning, not as an automatic 'proof' of curriculum coverage;

- teachers modelling writing and showing how it is constructed;

- using a variety of activities: at times these might be short, specific focused writing tasks; on other occasions they would allow time for boys to return to writing over a period of time; these activities would also mean a shift away from initial concerns about accuracy and neatness towards a sense of 'fitness for purpose' and knowing when it is important to 'get writing right';

- not engaging in purposeless writing - less writing, but writing which matters and which is relevant to the learners;

- more transparent assessment and marking with targets shared and negotiated with the pupils;

- having some sense of how literacy is perceived and supported at home.

In reviewing the triad's contribution to debates about raising boys' achievements in writing, significant success factors have been seen as related to the coherent management of learning at whole school and classroom level. This means an emphasis on longer term learning, not teaching alone and the importance of establishing a culture which values learners as individuals within an ordered learning environment with clear boundaries and high expectations.

\section{Case Study 3}

\section{The background}

The schools in this triad were situated in relatively prosperous medium-sized and small towns on the south coast of England. The Originator school was a larger than average primary school with over 370 pupils who came from a wide range of family and ethnic backgrounds. While the proportion of pupils eligible for free school meals was below average, above average numbers of pupils had special educational needs. Partner schools A and B were primary schools of average size, taking pupils from a range of social backgrounds, though very few pupils were from ethnic minority households. Partner school B served a large area of rented housing, and had a high level of mobility among its pupils; like the Originator school, it had an above average proportion of pupils who had special educational needs.

Although attainment on entry in all three schools was below average, results in National Curriculum tests at Key Stage 2 were in most years above average. At the start of the project results at Key Stage 2 in the Originator school had increased steadily between 1996 and 2000, with boys' results increasing at a slightly greater rate than those for girls, and a slight gender gap in favour of boys. In Partner school A, boys' results had increased markedly between 1997 and 2001, such that they out-performed girls overall in 2001, though the gender gap in English remained in favour of girls. Performance in Partner school B was more erratic from year to year, with girls' results in mathematics and boys' 
results in English fluctuating from year to year, but with a significant overall gender gap in favour of girls.

\section{Improving writing through drama.}

In October 2001, drama was becoming a focus for the local authority where the triad was situated. The drama advisor was proactive in getting schools and fellow advisors interested in a more creative approach in teaching literacy. Initial perception surveys in the Originator school, the headteacher's observations, indicated that the pupils may have had a limited diet of multi-sensory experiences both outside school and within the school curriculum. Drama offered a route into greater creative and sensory experience. Another head in the triad was particularly interested in the creative aspects of the curriculum and was keen to make cross-curricular links with literacy teaching based on the National Literacy Framework. In the third school, a rigorous schedule of assessing and setting targets for individual pupils in writing was already underway and the senior mangers were keen to follow the lead of the Originator school and explore the use of drama within the literacy hour.

In the first phase of the project, teachers wanted to combine the successful factors of the National Literacy Strategy (for example, termly objectives and a planned progression of skills) and the knowledge that a creative approach to teaching literacy may further enhance learning. In each school, drama was used to enhance all aspects of English teaching:

For reading, drama was used:

- to display understanding of the text;

- to reinforce understanding of the text;

- to gain empathy with characters;

- to work through higher order reading skills.

For writing, drama was used:

- to provide pupils with first hand experiences;

- to provide pupils with other ways of planning their writing;

- to allow pupils to work collaboratively on written tasks;

- to stimulate imagination and lead into descriptive writing;

- to provide an audience for writing by writing in role;

- to enable pupils to write for a range of purposes which feel 'real'.

For speaking and listening, drama was used:

- to enable pupils to discuss texts with their peers and teachers;

- to allow pupils to rehearse and 'draft' writing through talk;

- to formulate/plan compositions.

\section{Assessing progress in writing and drama}

During initial interviews the boys were generally negative about their own writing and reading. In common with boys in the second triad, in these initial interviews they mentioned handwriting, punctuation and spellings as factors that slowed down their 
writing but they also said that they were often worried about getting ideas for both fiction and non-fiction writing.

By the end of the first phase of this triad's work, case study evidence indicated progression in writing for all the boys involved. In all three schools the focus groups of boys achieved a higher standard of work during literacy activities involving drama. After a year's work, there was also a marked shift in their attitudes. Observations of the boys during drama showed them as enthusiastic, engaged and achieving in line with, or above, teacher expectations. When interviewed following drama work involving texts and writing, the boys commented:

I prefer doing drama in the class than writing straight away.

Drama helped me to write in the first person because it gives you an idea of what it would be like and their feelings.

Drama helps my writing because it gives me more information, it makes it (texts) more understandable.

In the Originator school each year group had established drama as an integral feature of literacy teaching units and was working with advisors from the LEA to raise awareness of the use of drama across the county. In the other schools, there had not been quite such an in-depth impact although several teachers had begun to integrate drama regularly into their literacy sessions. In evaluating the first phase, three main factors were identified as keys to successful implementation of drama as integral to literacy teaching:

- Teachers' subject knowledge about drama: the use of drama within the literacy hour relies heavily on the confidence, experience and knowledge of the teacher.

- Support: in-service training played a vital role in developing subject knowledge alongside school-based support with 'experts' within the school supporting less confident members of staff and the LEA providing county expertise.

- Allocating time for joint planning: dedicated time within school hours supported the success of the initiative.

This evaluation formed the basis for plans for a second, extension phase.

\section{The extension project}

The opportunity for another year's work allowed the triad to evaluate how far any gains by teachers and pupils were being sustained and how the approaches might be developed. The second phase aimed to look at how the established work on using drama to support writing could be embedded into the curriculum and extended to give other teachers confidence in using drama approaches. There was also the chance to strengthen links between the three schools, specifically by joint planning. One of the headteachers had moved school and was keen to continue involvement with the triad, hoping to develop the integrated use of drama in literacy in his new school. The group also decided to involve key stage 1 classes in all schools.

After a term's work there had been a mixed outcome to the collaborative work on planning. It was clear from the beginning that the year 4 teacher at the newly involved school was less enthusiastic than the headteacher and as the work progressed she felt unable to continue involvement. The year 1 teacher from this school also withdrew and so 
the year 1 group was disbanded. By the end of phase 2, however, the first three aims had largely been met in the two schools which remained involved. There had been some very successful joint planning sessions which had identified success factors and constraints for future developments of across-school planning and definite gains in the boys' achievements.

A final evaluative meeting considered: the evidence there had been for drama leading to better writing; classroom practices which have an impact on standards; and factors which support curriculum development related to drama (and writing).

\section{Evidence for drama leading to better writing: effects on writers and writing}

Teachers commented that there had been improvements in writing without using drama, but with it progress had been more noticeable. Attitudes and motivation had improved and the boys' confidence had increased. Progress was also evident in the pupils' language to describe writing (meta-language) before and after the work. One of the interview questions was specifically designed to measure development of meta-language: What advice would you give to someone in the year younger than you to help them get better at writing? Examples from the year 4 classes show that responses to this question in the initial interviews were mostly concerned with concentration, technicalities or the physical aspects of writing:

Stay calm. Take your time and keep practising at home

Use your guidelines properly. Always draw a margin on the left hand side so you know when to stop.

Always write in pen in literacy. Never write rude words in your book.

Use your guidelines or if you've lost them get a ruler. Try not to stick your elbows out and get jogged.

At the end of the project, the boys were noticeably more able to talk about writing specifically and in greater detail:

Teach them how to write neat, put in full stops. Tell them to imaginate (sic) yourself being in the book and you can work out what happens.

Show them your writing and see if they can get it a bit neater. I always used to press too hard. Relax a bit. Find a story for them - an easy story - and read it for them and they'll get ideas.

The boys were also aware of the link between drama and writing:

You get ideas about things from drama ... so you can come up with 'The Iron Man fell off the cliff very fast and left marks on the slide...'

Whenever you do drama and can't think of ideas and think you're someone and that you're dressed like them - and you are.

In one interview they talked about how role play had helped:

I was the mum. There was an argument and I looked angry and the teacher took pictures.

This sparked off a conversation about whether they minded taking on female roles. All of them were very positive that this did not worry them:

No, because you know you're a boy. 
Over one year, the boys made two thirds of a level progress judged by teacher assessments against National Curriculum level descriptors (which are written in the expectation of one level's progress every two years). The boys' writing showed clearer voice, structure and pace; vocabulary was being used more precisely and punctuation was more varied and consistently included.

\section{Classroom practices}

The teachers felt that it was important to establish a culture in the classroom which would support drama for writing. In this second phase of the project, the pupils were clearly getting used to classroom work which included drama as an expected part of literacy sessions. The teachers felt that they had become more assured in using drama themselves and that they could now involve the pupils themselves rather more:

We do a bit of drama then a bit of writing - fast - a diary entry - do it fast. Then you get the immediate effects of the feelings they've been exploring.

I can now change what I'm doing if I think it will help the writing.... Until you've done it enough you don't know what works but you may need a year or so... you can now pick out a bit and can use it in other curriculum areas.

You can justify what you're doing. We know that if it goes completely pear shaped it doesn't matter.

The school culture was also seen as important. Initiatives like this flourish best when the headteacher or senior management team fully support the work. However, the support of the head is not enough on its own, as the extension project has shown. There needs to be a developed culture of readiness to develop professionally. The school which withdrew from the extension project had simply not yet developed a culture which could support initiatives seen by the teachers as 'risky' and requiring them to question their own practice. As one of the teachers at the evaluation meeting commented: We were committed to it. That commitment is clearly a key factor.

\section{Factors which supported curriculum development: across schools}

The teachers valued working with a planning structure which was also flexible. In this way they were exemplifying the guidance in Excellence and Enjoyment (DfES 2003). The collaborative longer term unit planning integrating drama and writing had been very successful:

We planned it as a unit and interpreted it in our own ways. We were amazed at how much writing they'd covered.

We had a normal skeleton plan then applied our own activities.

We used the Literacy Framework and decided on a major outcome... The planning became emergent

They also felt that it had been helpful to work with a different school because this brought a new perspective. Also, seeing the results - the progress in the boys' attitudes and achievement that can be made in a relatively short space of time - gave them support.

The evaluation meeting wanted to describe the factors likely to lead to improved standards at local authority, school, classroom and individual level. The successes and problems of the project indicate some of the key factors for effective curriculum development in raising 
standards of writing through integrating drama with literacy. Clearly, it is important to establish a school culture which can support teachers as they take more curriculum 'risks', as they often see it, in bringing drama into a more central place in their teaching. This seems to be linked with the role of the headteacher. Where there is an established expectation and understanding, the head is likely to be in a better position to challenge teachers to develop their teaching approaches.

Decisions about which teachers might become involved are also critical. The most effective teachers were those who were already enthusiastic about developing their professional expertise by using drama. Once they felt more secure, they could then support others who were more tentative but prepared to try things out. These findings accord with success factors and constraints to creativity outlined in HMI's 2003 report on developing creativity in schools (Ofsted 2003b).

The steady embedding of drama into the curriculum is a matter of 'evolution not imposition'. This suggests that initiatives like this need at least a year of experimentation by one or two teachers in the school before involving others. The implications for curriculum development are clear: any initiative which means a fundamental shift of teaching approach will take time to develop with individuals before taking the approaches further and wider in the school (and, more significantly, across schools) and has to be made possible by senior management. In this triad another important factor in developing work across schools was the support from local authority advisers and in-service providers.

\section{Overview}

Throughout the project's main approaches, but perhaps most significantly in the literacy strand, key themes are: the importance of integrated, planned, systematic management and organisation - at whole school level as well as in classroom literacy teaching and learning; the valuable role of creative arts in the school curriculum and the effects of increasing confidence in learning. In terms of classroom teaching, in each of the three triads this was shown through clarity of teaching aims and processes; having high but realistic expectations; strong emphasis on group work; integrated planning; and teachers having a clear knowledge of pupils' abilities which allows them to provide for diversity so that they can challenge high achievers and support less assured learners. Some of these factors have already been identified in research into effective literacy teaching (Wray et al, 2002; Bearne et al, 2003). However, the single most significant factor which has not previously been specifically identified in work on effective literacy teaching to raise boys' achievements is the role of speaking and listening.

The Raising Boys' Achievement project has identified as critical both teachers' use of language and opportunities for pupils to extend their spoken repertoires. It has provided detailed evidence of the importance of systematic and clearly conceptualised oral work and identified some specific approaches to speaking and listening which contribute to improvements in boys' literacy:

Modelling the language of texts and of learning: teachers automatically using specific terminology about texts and language, as well as offering ways of thinking through their use of language, for example, Why has the author chosen to use those words? and What might be a way forward for this group? and thinking aloud: teachers sharing their thought 
processes and giving their own opinions, for example, I feel that this is more effective because... and I can see that working....

Asking questions - teachers and pupils: questions from teachers which are workfocused (rather than behaviour-focused) and vary between those requiring a precise response and those inviting reflection or speculation. The chance for pupils to ask questions is a significant contributor to boys' engagement with learning.

Talk during literacy sessions: deliberate planning for integration of all aspects of speaking and listening in each lesson including using drama within literacy learning.

In drawing together the different findings of the literacy areas of the project, it is clear that there are no easy answers. The schools involved are not offering quick fixes. However, their work has given some useful pointers for ways forward and raised some important issues about the crucial importance of an integrated approach to literacy and learning and one which specifically includes speaking and listening as a central feature. 


\section{CHAPTER 5: Pedagogy II: Issues about Teaching- Learning Styles}

A great deal of energy has been spent, over the last decade, in identifying pedagogies which are thought to be 'boy-friendly' and likely to be more effective in engaging boys in learning. Such lessons are defined as having a fast pace, a series of short and tightly focused activities, clearly defined and achievable aims and short-term targets. There is frequently an emphasis on competition, and on variety, structure and activity within lessons. Extensive classroom observations, across many triads in both primary and secondary schools, confirm many of these characteristics of effective lessons. We must recognise dangers, however, when we assume that such strategies are 'boy-friendly'.

There are several obvious problems in such a discussion:

- As we have suggested in chapter 1 , there is diversity and heterogeneity within this notion of 'boy' and 'girl', rather than homogeneity and uniformity; there is little evidence to suggest that 'boy-friendly' strategies suit most boys' learning styles and preferences, nor that these teaching methods are more appropriate to boys than to girls.

- Associated with this, there is sometimes an implicit - and in some schools - an explicit acknowledgement that girls do not need to be taught in similarly interactive and investigative styles, because they are often more cooperative and willing to learn, and will learn whatever teaching style is used. This is both demeaning and contestable.

- The concern with competition is particularly difficult. Whilst some boys may thrive in a classroom context which emphasises challenge, and be keen to engage in activities in which there will be winners (and conversely losers), so will some girls, and some boys will not be motivated in this way. Equally, though, there is something perverse in trying to engage 'under-achieving' boys, who have often failed to learn, in activities which, by emphasising the competitive element, expose them to the risk of further failure. Unless success can be assured in these competitive activities, there is the possibility of enhanced demotivation and disengagement of these boys.

- The concern with competition often runs counter, too, to collaboration. In some classrooms, it seems to us that girls perform better than boys because they use more interactive and cooperative modes of learning, with the emphasis on discussion and collaboration, working together on an issue or a problem, rather than in isolation, and competition with others. This ability to talk through an issue, to explore dimensions of the issue collaboratively with others, sustains and supports more effective learning, and needs to be mirrored by more boys.

In many schools, of course, there is a much more inclusive approach to teaching and learning, and there is increasing evidence to support the improving quality of teaching and learning. Nonetheless, these are important reservations as we search for more effective ways to engage students who might be under-achieving because the teaching they encounter is unfocused, lacking a challenge, dull and monotonous, without any excitement to motivate and engage. In such contexts, students felt that they wanted ... 
... more of a say in what we learn, how we learn and how teachers can get better. Teachers need to know about different learning styles, different ways to teach us, better and more varied teaching technique. We know it's hard for some teachers, but they need to help us structure our learning more.

and, in the words of the headteacher of one Originator school:

There's no point doing all the work we do on reducing absentee and exclusion levels unless we transform what's happening in the classroom, so that they feel that what's on offer is worth coming for!

\section{Teaching - Learning Styles}

One aspect of the pedagogic work of the Project has focused, through two secondary triads and one primary triad, on learning styles work within the context of accelerated learning (Smith, 1997, 1998) and on exploring the application of Gardner's work on multiple intelligences $(1983,1999)$ to classroom practice. Smith's work (Smith 2001a), on 'brainbased' accelerated learning in practice (ALPS), aims to enhance pupils' motivation and achievement, and is based around the notion that pupils can be taught to think and learn more effectively using a range of visual, auditory and kinaesthetic techniques (VAK), such as mind-mapping, musical stimulation, physical activity and practical design activities. Gardner's notion of multiple intelligences identifies a cluster of distinct intelligences, grounded in neurobiology, psychology and anthropology; initially a set of seven (linguistic, musical, logical-mathematical, spatial, bodily-kinaesthetic, interpersonal and intrapersonal), these have subsequently been extended to nine with the additional identification of naturalist and existential intelligences. Gardner's work has likewise been taken up by schools and local education authorities because it has seemed to acknowledge students' varying strengths and predispositions, and it has been perceived as a way of structuring school and classroom experiences to promote learning.

There has been active promotion of in-service training and professional development on learning styles, for both secondary and primary schools, particularly by some local education authorities, and implicit endorsement by the DfES itself, both through its website on learning styles and brain-based learning (DfES, 2004c), and through the resource materials produced by London Challenge and the Key Stage 3 Strategy, Ensuring the Attainment of Black Caribbean Boys (DfES, 2004d). In each of these three triads, work on preferred learning styles had impacted markedly within each Originator school, and there was some confidence in the potential of the work to enhance boys' and girls' motivation, and their subsequent achievements in public examinations.

\section{Case Study 1: Accessing Kinaesthetic Learners more effectively}

The Originator and Partner schools within one triad were all 13-18 upper schools in different local education authorities in East Anglia and the East Midlands. Each school served the local market town in which it was located, together with an extensive rural catchment. The Originator school had previously been involved in a number of regional and LEA based projects involving the development of learning styles based upon work on accelerated learning and multiple intelligences, and there seemed some evidence, from its GCSE data through time, that this work had impacted upon students' performance data, with an upward trajectory of results and a corresponding narrowing of the gender gap. 
Our initial work within this triad brought some surprises. Different instruments, used in different schools to analyse students' dominance profiles (the balance between visual, auditory and kinaesthetic preferred learning styles), yielded different results, raising the intriguing possibility that students in broadly similar schools had significantly different learning styles from each other. Subsequent use of a common questionnaire ${ }^{1}$ produced broadly comparable results across the three schools, however, and reiterated the need for care in the choice of the instrument to be used. Equally, analysis of the learning dominance profiles of 384 boys and 335 girls did not reveal any correlation with gender: indeed, contrary to assertions in some of the literature, the mean scores suggested that, if anything, there was more of a kinaesthetic tendency amongst the girls than the boys. Further analysis in one Partner school, however, revealed that those students (mainly boys) characterised by teachers as falling into one of a number of 'bad behaviour' categories and in danger of 'under-achieving', (for example, those said by teachers to be 'disillusioned and negative about teachers and school' or those who were 'disorganised') did record a dominance profile which emphasised kinaesthetic learning. 'Model' students, in contrast, and both male and female teachers, displayed a marked tendency to visual learning.

Lesson observation data, derived from student shadowing exercises in each school in the triad and subsequent interviews with key students, confirmed that very few lessons in 'traditional academic' subjects gave opportunities for students to learn kinaesthetically, compared with subjects such as the Arts subjects, the various Technology options, prevocational courses, Graphics and ICT, where kinaesthetic learning activities were dominant. In each school within the triad, however, pockets of innovative kinaesthetic practice were identified, and the process of developmental work within the triad focused on identifying the core of this effective practice. This led to a classification of kinaesthetic activities, with emphasis - for example - on those activities which involved students in handling objects, artefacts and models to support learning; drama and role-play; investigative work using student movement and / or experimentation; the active use of ICT to support learning; the use of movement, place and space to change atmosphere and refocus students' minds; the use of movement in space to capture and reinforce ideas and terminology.

These school-specific teaching approaches were brought together in a teaching activities booklet, common to all the three schools, and presented to staff development sessions in each school. Staff were encouraged to introduce a range of these kinaesthetic activities into their teaching, and - through monitoring their use of such strategies - to evaluate their effectiveness in supporting the learning, motivation and engagement of their classes and of particular groups and individuals within their classes. The research process in subsequent years, as the students progressed from year 9 through to year 11, focused on refining and extending the range of kinaesthetic teaching activities, in the light of experience in each of the triad's schools, and monitoring staff and students' perceptions of the effectiveness of the strategies.

The developmental process in this triad was firmly rooted in the realities of existing pedagogic practice within each school; teachers evolved their own classroom situations rather than approaches being 'top-down' or imposed from outside 'expert' practice, and

${ }^{1}$ From O'Brien, L (1991) SOS: Strengthening of Skills (Rockville MD: Specific Diagnostic Studies Inc) 
thus they developed a sense of ownership of the work which the triad was developing. By the very nature of their evolution, therefore, the range of these pedagogic strategies which evolved to support kinaesthetic learning in these schools was not new, untried or experimental. In Religious Education and History, students were encouraged to move around the classroom in carousel style to explore different types of resources - artefacts, CD-Rom, illustrations, decoding documents and symbols. Investigative work in science and Maths involved mind mapping, brain gym activities and physical sequencing. In geography, jigsaw activities were used as part of role play, and students were encouraged to use ICT publisher programs to design leaflets and poster presentations. In English, emphasis was placed on strategies such as hot-seating, analysing and subsequently enacting short video clips, and creating themed illustrations to draw out aspects of poems and set books. The second edition of the resource booklet on kinaesthetic learning activities thus drew on current existing practice in all the schools, and offered examples of over 250 subject-specific and generic proposed learning activities.

In parallel, it was recognised that this process of developing kinaesthetically-orientated pedagogic activities needed to be complemented by work with students, to make more explicit for them the ideas associated with preferred learning styles. Sessions on study skills, learning styles and revision techniques were incorporated into the Pastoral, Social and Health Education (PSHE) programmes in each school, and keynote sessions taught to all students. Feedback on 'VAK testing' (visual, auditory and kinaesthetic testing) also enabled all students to become aware of their own dominant preferred learning style, and the balance between the visual, the auditory and the kinaesthetic in their own individual profile.

\section{Impact}

Tangible outcomes from the work in this triad are mixed. Teachers were asked to review their use of kinaesthetic teaching approaches at the start and end of the final year of the project, and their self-review - across the three schools - suggested that there had been little increase in the use of kinaesthetic activities. Students' perceptions, analysed at the start and end of their GCSE course, over six terms, confirmed that, at the outset of the research, much of the teaching in high status, 'traditional academic' subjects in all three schools placed more emphasis on auditory (English, French, History) and visual (Mathematics) modes of learning, with only teaching in the sciences approaching more of a balance in the learning styles encountered by the students. This pattern remained unchanged throughout their GCSE courses for students in two schools, but in one Partner school, there appeared to be a marked increase in kinaesthetic activities so that students perceived that teaching was reasonably balanced across the three modes of learning in English, Science, History and Geography, with kinaesthetic activities predominant in Mathematics. When data are analysed in gender terms, there is little difference in response between boys and girls in any of the three schools; in the Partner school where the balance of teaching activities is perceived to have changed, this observation is offered by both girls and boys.

One of the difficulties encountered in this triad, however, was trying to identify, with any degree of specificity, the extent to which the use of kinaesthetic teaching activities had actually changed over a limited time span. Questionnaire returns from teachers themselves tended to be inconclusive on this issue; whilst some of the original strategies were more 
prominent in some schools, other strategies were apparently used less frequently. At one level, the outcomes of the research in this triad suggests that, over a limited time period, it is difficult to change the balance of teaching activities and teaching styles used by teachers. In most of the high status academic subjects, there has been little evidence, except in one Partner school, of any marked increase in teaching activities which access kinaesthetic learning, and teaching which accesses visual and the auditory modes of learning continues to dominate. In part, this may be because, whilst teachers did acknowledge the importance of introducing more kinaesthetic learning activities, in order to present more of a balanced range of learning opportunities across each learning style, some teachers did not feel they had received sufficient guidance in adapting the teaching activities listed in the resource booklet for their own subject and context. Teachers suggested, for example, that they would have welcomed the opportunity to observe the practice of colleagues who were more experienced and confident in using kinaesthetic activities, to have had subject-specific staff development time to formulate, prepare and subsequently evaluate pilot activities, and to have had focused in-service activities to enable them to explore the detailed implications for implementing such approaches, and incorporating them into departmental medium-term planning.

At another level, however, the outcomes in this triad were more encouraging. Senior staff who had coordinated the research in each school reported that the project had impacted very positively on teachers' awareness of how students learn, and had focused staff concern on the learning process, for themselves as well as for students. They also suggested, on the basis of their extensive experiences of classroom observations across different subjects in their own schools that there had been some significant changes, particularly in the increased use of kinaesthetic activities in middle ability and lower ability groups, and in the quality of kinaesthetic activities used by staff. This issue of quality is interesting. It was felt that teachers were now far more aware of the variety of kinaesthetic activities which were appropriate in classrooms, and how these actively supported real learning. There is a degree of subjectivity here, perhaps, but discussion with these senior staff does suggest that there is more thoughtful use of kinaesthetic activity, more evaluation of their effectiveness and more integration into the structure of lessons on an ongoing basis, rather than reliance on high profile, infrequent events which might support learning less effectively.

\section{Case Study 2: An approach based around multiple intelligences}

The second triad was based around secondary schools in the West Midlands which served mixed catchment areas, with predominantly white working class intakes, although in each school there was a significant proportion of students of Indian or Black Caribbean heritage. Areas of extreme social and economic disadvantage existed within each catchment area, and each school exhibited - to a greater or lesser degree - features closely associated with schools in challenging circumstances. The Originator school was in 'Special Measures' for a time six years ago, with only $84 \%$ of its student capacity filled; it received a significant number of students excluded from other schools, as well as children from households displaced to the large local authority estates around the school by family breakdown, insecurity of the job market and high debt levels. The Partner schools were similarly under-subscribed, with significant weaknesses identified by Ofsted in the late 90s, particularly within the context of pedagogy, low expectations of students and insufficient challenge to motivate students. 


\section{The Nature of the Intervention}

As in the first case study, a strong interest had developed in the Originator school in accelerated learning and multiple intelligences. Work on students' preferred learning styles had been researched by senior staff and was initially developed with students in terms of identifying preferred learning styles, through a focus on multiple intelligences. Parallel staff development sessions and lesson observations focused on identifying teaching strategies which were differentiated to target different preferred learning styles.

Work with the RBA project focused, in two successive years, on developing this approach further within single-sex groups, focusing initially on higher ability boys in year 9 who were perceived as being potential under-achievers in English. Identification of the preferred learning styles of the first cohort of boys revealed that a number of preferred learning styles - mathematical / logical, inter-personal, linguistic and visual / spatial intelligences - were more dominant than others in these boys' profiles. Amongst the second cohort of boys, mathematical / logical and kinaesthetic learning styles were dominant. This difference between the boys in the two cohorts is not surprising given the small sample size, but again there is a warning here in case we assume that any class of boys and girls will have predominant preferred learning styles which are gender determined.

With each cohort of students, the intervention strategy then focused on how to transform classroom practices, initially to accommodate these preferred learning styles. With the first cohort, the focus was placed specifically on the mathematical / logical and inter-personal learning styles because teachers felt that these were less well-developed in the teaching of English. The classroom pedagogy thus required the boys to work together in small groups and teams, on jigsaw activities which focused on group interactions and presentations, on role-play activity, and on drama. In accessing mathematical / logical learning styles in the teaching of Macbeth, the boys were involved in short, sharply focused activities which emphasised ordering ideas, sequencing events, developing a train of thought based on evidence from the play, and making predictions and analysis as a result of collating given information.

In the second year, emphasis was also placed on developing teaching styles which stressed intra-personal ways of learning, since teachers felt that this was a marked weakness which restricted the boys' learning in English. In fact, this had not been identified as a preferred learning style by any of the boys. So teachers devised teaching approaches which enabled the boys to visualize better the activities which were being enacted within the text, and to formalise their feelings and responses to incidents within a particular play. The boys were particularly encouraged to reflect upon the range of emotions and experiences which the play was creating, through talking with each other in small groups, and asking questions of each other, to help in their search for understanding.

The research process in this Originator school changed focus over the two years, then, from emphasising boys' preferred learning styles, to focus subsequently upon both strengths and weaknesses in preferred learning styles.

\section{Outcomes in the Originator School: Patterns of Achievement}

With both cohorts of boys, attendance at school improved and temporary exclusion rates dropped; lesson observations conducted by senior staff within the school suggested that the 
boys were more motivated and involved in their work than earlier in the year. There is also some evidence of the favourable impact of these initiatives on learning and achievement, although this needs to be treated with caution. In the National Curriculum English tests in year 9, the performance of the first cohort of boys was less strong than that of the corresponding cohort of girls; overall they achieved a mean attainment level of 4.96 compared with 5.44 for the girls. The boys scored a higher mean mark than the girls on the 'Shakespeare' paper, at which the strategy was directed, however, and a higher mean mark on the 'Shakespeare' paper than other aspects of the key stage 3 English tests. In the second cohort, boys did less well overall and on the 'Shakespeare' paper than the corresponding cohort of girls, but the boys' performance was better on the 'Shakespeare' paper than in other aspects of the examination. We need to be extremely careful in extrapolating firm outcomes from such a limited context, but one conclusion may be to suggest that the boys were able to identify with the modes of teaching developed by the teachers for their study of Macbeth, but were unable to transfer their emerging understanding and skills as a learner to facilitate their learning in other aspects of their English work.

\section{Outcomes in the Originator School: Students' Views and Perspectives from the staffroom}

Although the focus of the research in this school was on a range of teachers, it is still difficult to separate the impact of the intervention strategy itself from issues to do with teacher personality and charisma. Nonetheless, it is possible to draw a number of tentative conclusions from longitudinal interviews with boys in both cohorts carried out over a three year period:

- the boys interviewed often had a clear understanding of basic ideas about preferred learning styles, knew their own dominant style and were able to talk about how this related to their learning;

- usually, they knew the dominance profiles of some of their teachers, since these were displayed prominently in classrooms and offices around the school;

- some boys suggested that their preferred learning style might change through time;

- some boys were beginning to become aware of their own strengths and weaknesses as learners, and were able to discuss with each other how they learnt best;

- they knew that some lessons used a variety of different learning styles within them, and that some teachers made explicit reference to preferred learning styles within lessons.

Their reaction to the teaching of Macbeth was unequivocal. The variety of the teaching the use of video, of text reading, of listening to tapes, of acting and drama led by the teacher himself - was crucial to its success, and the fact that the boys knew that the lessons were carefully planned and organised to access a variety of learning styles meant that there was a sense of being in a collaborative learning enterprise with the teacher. Interviewed again as year 10 students in May, the vividness of the activity had remained with them. They described the real difference which it had made to their learning, the stimulus which had been provided by the variety of teaching styles and the explicit link to learning, of the excitement and activity of the lessons: 
The way he taught, it really helped us to remember scenes from play. It made English lessons different, so enjoyable, like active.

Yeah, like when he kept changing things around, so we did lots of things which involved acting, watching a video of the play, listening to music to get at the mood of the play.

And he used rhythm from songs we knew to remember key points and quotes.

It's been so brilliant; I didn't think I could understand a play like that.

This work on preferred learning styles pervaded much of the staff development work on pedagogy within the Originator school, and was linked into the staff peer-review process. Whilst it would be an over-statement to claim that this approach to pedagogy had been adopted by all teachers, it was clear - from classroom observations and students' interviews - that it was high profile in a number of departments, notably in Science, Mathematics, Modern Foreign Languages and Music, as well as in English. Teachers in these departments felt that the approach gave them opportunity to be more creative in their planning and teaching, and enabled them to be more analytical about the learning teaching process.

As our interviews with students confirmed, central to this work was the sharing of the notion of preferred learning styles with the students. Teachers continually engaged in dialogue with the students, to make them aware of the preferred learning styles being accessed in a particular lesson, and to reassure them that their own lack of understanding or difficulty with a topic was not linked to any inherent shortcomings of their own, but might be linked to the nature of the learning demanded or the way in which the topic had been taught. In the words of a senior member of staff:

I want students to be able to say to me: 'I don't understand that; can you teach it to me in a different way please?'

\section{The Intervention within one Partner School}

Work on preferred learning styles and on multiple intelligences was not high profile in either Partner school at the outset of the intervention stage of the project, and one Partner school did not become fully involved as time progressed. In the second (11-18) Partner school, although there was strong support in principle from the headteacher, initial involvement with the RBA project in the whole school was also limited in scope. However, the opportunity to be involved in developmental work on teaching-learning styles was seized upon by the Mathematics department in this school. Here, developments reflected the initiatives taken in the Originator. Pedagogic approaches relating to each multiple intelligence were identified and exemplified, and resources made available, for example, to support activities based around mind mapping, role-play and effective groupwork. Booklets were produced for Year 11 students, outlining teaching strategies which teachers might use to access different intelligences, and recommending revision strategies which might help to consolidate the learning of different types of learner.

Subsequent interviews immediately prior to their GCSE examinations revealed that Year 11 boys were strongly enthusiastic about the quality of teaching they received in Mathematics: 
I really enjoy Maths because there is more variety: we use rhymes and colours to understand formulae, we have classical background music to create a calmer atmosphere which is more soothing and helps concentration, we have kinaesthetic activities which involve relays and board work and lots on movement and activity.

She does more interactive things with us, uses whiteboards, uses games and bingo so that we learn new ways of looking at and doing Maths, she uses humour and wears silly hats, she teaches in such an interesting way, it's impossible not to learn.

In Maths we don't just sit there as though we're dead - we get on very well with her because she trusts us and works hard for us, so we return the trust. If she says we're to do something which seems really weird because it will help us learn, then we do it!

Subsequently, the scope of the work was broadened into other subjects and into whole staff continuing professional development activities. A core group of interested staff was established, involving representatives drawn from Humanities, Mathematics, Physical Education, Art, Modern Foreign Languages and Science, to try to establish a coherent base from which diffusion might subsequently take place across a wider group of staff.

Interviews conducted with Year 11 boys and girls during the second year of the Partner school's involvement with the RBA Project suggested, however, that - beyond Mathematics - the learning styles initiative was not yet impacting on their consciousness in any real way. In some respects, these outcomes were not surprising, given the recent introduction of the initiative to the whole staff, although we had anticipated more positive feedback! To some degree, students could identify the style of teaching associated with effective teaching, but were unable to go beyond this to discuss their own learning styles in anything other than the most general of terms. Although some could identify their preferred learning styles, fewer could articulate what this meant for them as individual learners. There was little sense yet of how they might help their own learning, or of the need to take effective responsibility themselves for their own learning. In this context, then, there was less emphasis placed - by teacher or students - on the students' own preferred learning styles; the focus instead was on the broader pedagogy, on a variety of innovative, lively and entertaining teaching methods, to stimulate and aid learning.

In terms of whole school impact, then, it is clear that the intervention work in this Partner School was only at an early stage of development, accepted and adopted by an enthusiast who was herself a charismatic and dedicated teacher, but not yet engaging the whole staff.

\section{Case Study 3: A Primary Context}

The primary schools which worked on learning needs and teaching styles were located in mixed urban / rural catchments in one local education authority in East Anglia, where the LEA had been proactive in stimulating discussion of ideas associated with multiple intelligences amongst its schools. There was a strong commitment, therefore, in the Originator school (although not in the Partner schools), to the promotion of multi-sensory approaches to learning which incorporated references to visual, auditory and kinaesthetic styles of learning and, in the view of the staff, this had impacted positively on boys' and girls' motivation and attainment at key stage 2. At the start of the project, data from the Originator school (a two-class entry school) showed a steady increase in the Key Stage 2 performance of boys and girls in English between 1996 and 2000, with a narrowing gender gap, such that the gender gap in 2000 was negligible (see Table 5.1). 
Table 5.1: Average points scores in KS2 National Curriculum Tests, Originator School

\begin{tabular}{|c|c|c|c|}
\hline Year & $\begin{array}{c}\text { Boys } \\
\text { (Average points } \\
\text { scores) }\end{array}$ & $\begin{array}{c}\text { Girls } \\
\text { (Average points } \\
\text { scores) }\end{array}$ & Boy:Girl Ratio \\
\hline 1996 & 25.35 & 26.80 & .95 \\
\hline 1997 & 24.66 & 27.60 & .89 \\
\hline 1998 & 26.00 & 27.75 & .94 \\
\hline 1999 & 28.23 & 29.21 & .97 \\
\hline 2000 & 29.77 & 29.86 & 1.00 \\
\hline
\end{tabular}

Performance in national curriculum tests in this school remained above the national average, and gender parity between the performance of girls and boys in English was maintained through the lifetime of the project. Inevitably, though, average points scores varied from year to year, as would be expected given the small size of the cohorts.

It was not difficult, in each of the three schools, for teachers to identify some year 5 boys who appeared, from the quality of their work or their classroom engagement, to be underachieving when compared with their performance at Key Stage 1. Observations conducted by senior staff in each school during lessons and at playtimes suggested that these boys were frequent non-participants in class or group discussion, often misinterpreted tasks set by the teacher, were passively disengaged in lessons, yet were assertive and quite dominant within the peer group when out of lessons.

The research design within the triad incorporated a number of different methods:

- establishing a small focus group of 'under-achieving' boys in each school, and identifying, through questionnaire and response to pictorial stimuli, the preferred learning styles (plural) of each of these boys;

- confirming these learning styles through individual interviews with each boy, to provide each boy with more self-awareness of self as a learner;

- staff development work on preferred learning styles, in association with the LEA, to enable teachers to develop activities which would differentiate learning, cater for pupils' strengths and preferred learning styles, and develop strategies to support pupils to work more effectively in their 'less preferred' styles

- a more integrated approach to curriculum planning, to enable Foundation subject resources and teaching approaches to support the teaching of the core subjects.

- tracking the boys' attitudes, behaviour and attainment over a two-year span, through years 5 and 6 , to make an initial assessment of the impact of the intervention strategy

- at the conclusion of the two-year cycle, interviews with teachers and lesson observations carried out by members of the research team 


\section{Impact}

While one of the Partner schools decided not to proceed with the chosen strategy into the second year, end-of-project interviews with focus group boys in the other two schools suggested that they were shifting their previously negative opinions about school, and felt that teachers were more proactive in helping them access learning that they were finding difficult. The boys commented, for example, that:

She helps us to do better and gives us choices.

She asks us if we understand and makes it fun.

He uses picture cards to help me.

We use whiteboards to work out sums the quickest, it's bigger than a page.

He points carefully to the things I need to look at.

And there were more explicit references to modes of learning:

I like acting things out.

He shows me how to do stuff, I learn by looking.

They set up activities and they know what we like.

It was clear that these boys had a better understanding of how teachers were trying to support their learning, through role play, through group work, through carousel activities which involved searching the classroom for prompts and clues, through drama, through scientific experiments:

In geography, we had to make our own city in Egypt that would be better than Cairo.

We done more talking about Twelfth Night.

In PSHE I got to be the teacher. I learnt how to lead a group.

Interviews with teachers, and lesson observations at the conclusion of the two-year cycle, suggested a number of significant changes within each school:

- teachers planning and teaching in more cross-curricular ways, combining art and literacy, for example, or ICT and history, or science and literacy, to diversify the range of learning styles in lessons;

- teachers' increased awareness of preferred learning styles of individual pupils, differentiating to build on strengths and establish confidence, and offering support to develop other, less favoured aspects of pupils' learning;

- more pupil engagement within lessons, with increased motivation, and a better understanding of the intentions of the teacher.

In terms of value added data, the focus group boys in the Originator school in general did not do as well as might have been hoped, with four of the eight boys under-performing in terms of their progress between Key Stages 1 and 2. However, during the school's work with the project, there was evidence of gains made between the end of year 5 and the end of year 6, with four of the focus group boys making average progress and two making better progress than expected. In the Partner school, too, some progress was apparent between teacher assessments at the beginning of year 6 and final national curriculum test 
results (Table 5.2) particularly in reading, where seven of the nine focus group boys made greater progress than the average one-third of a level.

Table 5.2: Progress in Partner school during Year 6 in relation to National Curriculum levels (no. of boys)

\begin{tabular}{|c|c|c|c|}
\hline & $1 / 3^{\text {rd }}$ Level & $2 / 3^{\text {rd }}$ Level & 1 Level \\
\hline Reading & 2 & 4 & 2 \\
\hline Writing & 5 & 3 & \\
\hline
\end{tabular}

The work with this triad was extended into a third year, and involved tracking the focus group boys from two of the primary schools into their first year of secondary education, and comparing their experiences with a similarly sized 'control' group. Again, the small sample size means that it is unwise to suggest that conclusions can be drawn which have widespread validity. Within these groups of boys, however, in two contrasting secondary schools, the boys who had experienced the learning styles approach in their primary school were more able to express ideas about what helped them to learn and what made learning difficult. They were also more likely to be able to talk about generic activities than to talk about specific subjects, and there seemed to be less gender stereotyped responses concerning learning from these boys than there had been when they were at primary school. This suggests that in this context the learning styles approach may have had a positive effect in helping the boys to think about their own learning.

Research within this triad suggests that work on preferred learning styles within the primary context needs to be firmly located within a whole school context, cross referencing to integrated curriculum planning and to broader pedagogic issues. The central role of the class teacher in many primary schools means that the ethos and environment of the classroom is crucially important, too, because pedagogy and teaching style cannot be divorced from the overall tone and implicit messages which the children receive. It is important, too, that VAK (visual, auditory, kinaesthetic) activities are planned to occur together rather than separately. There appears to be a need to combine them, since many pupils wanted to be able to listen and look at the same time, and felt that they learned better when such an approach was used. More interactive learning styles, increased opportunities for groupwork and for talk to support learning, more physical breaks and opportunities for activities such as brain gym, will not be effective in isolation. All of these activities can access learning for more children, whatever their preferred learning styles, but teachers need to support this by developing a classroom culture which encourages children to take increased responsibility for their own learning and to become more independent in their approach to learning.

\section{Reflections on Pedagogic Approaches Focusing on Preferred Learning Styles}

There has been some very interesting work published on learning styles, both in whole school contexts (Wise and Lovatt, 2001) and in terms of research into teacher effectiveness (Hay McBer, 2000). To date, though, there has been little empirical work 
which supports some of the claims made by protagonists of accelerated learning and multiple intelligences. One notable aspect of Smith's work, for example, is that whilst it clearly offers a wide range of practical strategies which have undoubtedly been welcomed by some teachers, Smith himself acknowledges that

This work is not research-based. It is pragmatic and based on detailed elicitation and modelling. (Smith, 2001b, $p$ 173).

Indeed, recent research (Coffield et al, 2004) is somewhat critical of the use made in educational contexts of various models (such as Dunn \& Dunn's learning style model, and Gregorc's style delineator) which are based around the four modalities (visual, auditory, kinaesthetic and tactile), and underpin much of Smith's work on accelerated learning. It is perplexing, too, that so much of the work on preferred learning styles appears without context or critique, and the assumption is conveyed that these are valuable tools, which can be implemented quickly and uncritically, to extend the range of teaching which these students encounter. The learning styles movement has thus gained both a self-generating momentum and a rather uncritical golden halo effect.

It is within this context of competing claims - pragmatic insistence on the effectiveness of learning styles work in schools and theoretical misgivings about the validity and independence of the claims made - that we have situated our work with schools in these triads. In so doing, we wanted to support the development of teaching and learning strategies which focused on accelerated learning and multiple intelligences, and at the same time to identify an evidential base, where possible, to evaluate critically some of the claims made about learning styles.

Our work with these triads of schools, in very different socio-economic contexts, raises for us some important issues about teaching and learning:

- there is a need to acknowledge the difficulty of measuring and identifying students' preferred learning styles, and to recognise the different clarity and validity of different instruments which purport to measure this;

- there are very real difficulties in defining the learning styles which are accessed by different classroom-based activities, since many of these activities involve all three modalities;

- the approaches in some schools try to identify the preferred learning style (singular) of a student, without contextualising this within a balanced approach which looks at the significance of each learning style within the overall dominance profile. To do so can often exacerbate barriers to learning, rather than alerting students to opportunities;

- activities planned by teachers to access a particular learning style may well be processed by students in quite different ways;

- learning styles are flexible and can change through time, in response to different styles of teaching and to learning opportunities.

These are important reservations which need to be highlighted because work on preferred learning styles is very much in vogue but is sometimes implemented in simplistic and mechanistic ways. Nonetheless, research within these case studies does tend to suggest, albeit to a greater degree in some schools than in others, that work based on preferred learning styles and notions of multiple intelligences can help to enrich the learning of some 
boys and some girls. Where this work has been most successful, there has been an explicit focus on pedagogy and on teaching styles, not simply on students' learning styles. As we have seen in different schools, student engagement and learning has been enhanced when pedagogy has been opened up and discussed with students, when learning has been made more accessible to students, and when varied and stimulating approaches to teaching have been developed. These students get to the heart of the matter:

\begin{abstract}
Some teachers open up, talk with you about how you learn and how they're trying to teach. It makes it so much clearer, you can see what they're trying to do, and you realise all the thought that's gone into the lesson, so that makes you think you'll work harder too, in return.

We enjoy the work, don't we, because we're not copying from books or just writing down notes that the teacher is parroting. The lessons are active and practical, discussions, experiments. We get the feeling the lesson has been prepared carefully and just for us, for us to learn. She's safe, and she only has a go at us when we need it.

I like all this stuff about preferred learning styles ... it helps me to be more confident, to try to work things out for myself, and then to ask if I don't understand.
\end{abstract}

\title{
Important Pre-Conditions
}

There are, then, a number of enabling factors which need to be established if work on preferred learning style and pedagogy is to be most effective:

- raising awareness of how learning takes place, through keynote presentations to staff and students about different modes and styles of learning;

- consolidating and developing this through a tutorial or PSHE programme which focused on appropriate study skills, and how students might acquire different study skills for different contexts;

- emphasising the implications of knowing about preferred learning styles: the crucial importance of realising that they would only learn when they could access learning styles which were not their natural preferences;

- planning lessons which explicitly addressed a variety of preferred learning styles, and enabling teachers to become more creative in their teaching, planning and assessing;

- establishing the initiative with a wide range of staff, to provide some measure of consistency of expectation for the students, and ensuring that the gains achieved in terms of students' attitudes, engagement and motivation - were sustained as they progressed through school;

- regularly re--assessing students' preferred learning styles, and enabling teachers and learners within schools to keep the issue high profile. Indeed, one of the key informants in the first case study suggested that his preferred learning style differed from context to context, depending on the focus of the lesson.

When such pre-conditions exist, students have more opportunity to develop a better understanding of themselves as learners. In our experiences, the essence of work on preferred learning styles is not to define and indulge a particular learning style (because such categorisation of students is inflexible, may often be dangerously misleading and may 
exclude some students altogether), but to emphasise a balance of different learning activities and teaching styles, and through these activities, to recognise and value diversity of need. 


\section{CHAPTER 6: The Context of the Individual: Target- Setting and Mentoring}

There are few secondary schools in England which have not introduced some form of mentoring and target-setting. A number of initiatives (for example The National Mentoring Network, Excellence in Cities and Connexions), set out to recruit both paid and unpaid mentors to help support the learning and continuing involvement in education of young people at times of crucial transitions in their lives. There is, as a consequence, a considerable literature on mentoring which offers schools guidance, which suggests that mentoring schemes need to:

- have a clear purpose (Sims, 2002) and achievable aims which are communicated to all participants (Reid, 2002);

- be formalised within a clear context of where and when it is to take place (Hirom, 1999);

- have clear criteria for identifying mentors and mentees (Sukhnandan et al, 2000);

- be supported by training for mentors, to ensure knowledge of process as well as content of what the meetings should cover (Morrison, 2000), and to facilitate appropriate listening skills and body language (Radice, 2001, Pyatt, 2002);

- be non-judgemental and understanding (Reid, 2002), with a balance between tutoring, guiding and counselling (Hirom, 1999).

In some contexts, it is clear that mentoring schemes have been developed which have helped students address weaknesses and improve their learning skills, reconciled career aspirations and necessary short-term academic targets, and identified strategies which have helped students to meet those targets. In other contexts, however, the outcomes have been less encouraging, with students' resistance to change, a refusal to engage with targetsetting and mentoring, and an outright rejection of offers of support.

Thus whilst the vast growth of mentoring schemes within the last decade has involved an extensive commitment of time and energy by teachers, support staff and volunteers, it is often not clear if, how or why mentoring contributes to raised academic achievement. Thus there is, according to Colley:

'... little evidence to support the use of mentoring on such a vast scale.' (Colley, 2003, p 523).

\section{Target-Setting and Mentoring within the Raising Boys' Achievement Project}

The schools which have worked within the Raising Boys'Achievement Project on initiatives related to mentoring and target-setting have done so from differing perspectives and within different contexts. In one triad of schools mentoring has been an explicit and direct intervention, introduced formally in the final term of year 10, towards the end of a student's period of compulsory education. The system has embraced all students, regardless of gender or ability, and mentoring has been carried out by senior members of staff - including the headteacher in each school - who have made a considerable time commitment to support the learning of students. Mentoring sessions have been regular and frequent, normally on a monthly basis throughout the last year of 11-16 education, and 
have been informed by a rigorous system of data collection and analysis which has been fed by each subject department within the schools.

In a second triad of schools there has been more concern with developing a mentor scheme directed at the needs of students in key stage 3 (years 7-9). Here a smaller number of specific students have been targeted, on the one hand those students (usually boys but with an increasing number of girls) who have been identified as being 'at risk' of failing to fulfil their potential, and on the other, a number of students identified as 'hidden underachievers', those girls and boys classified as low achievers but not qualifying for special needs support, who the school feels are capable of performing at a higher level. In this second triad of schools, mentors have been either Learning Mentors, funded by initiatives such as Excellence in Cities, or teachers who usually do not directly teach the students they mentor, chosen for their personality and their ability to establish rapport with more challenging students. In this triad, too, mentoring has been regular and frequent, within protected time for the student, and informed by data on behaviour, effort and quality of homework, as well as by attainment data.

The contrasting characteristics of mentoring and target-setting in these two triads reinforce some of the dilemmas associated with such interventions. Given limited resources of time and energy, where should such interventions be located? Who should be targeted? Who should mentor? What are the success criteria for such interventions?

\section{Case Study 1}

\section{The background:}

The schools in this triad were 11-16 comprehensive schools on the periphery of urban areas in North-East England, serving essentially white mixed catchment areas of rural agricultural and commuting villages, together with large local authority housing estates within each urban area. Parts of each catchment contained areas of significant social deprivation and exclusion, with a history of low parental aspirations within the parental community. The nature of the catchment areas, and the size of the schools, had remained stable throughout the last decade.

The Originator school had an unexceptional achievement profile through much of the 1990s. The Ofsted report of 1998 summarised the pupils' achievements as being 'broadly in line with those expected nationally of pupils by the end of KS3 and KS4 ... and similar to those of the last inspection (in 1993)'; in $199739 \%$ of boys and $42 \%$ of girls achieved $5(+) A *_{-}$C grades at GCSE. Since then, however, an exceptional transformation in achievement has taken place (see Table 6.1), not only in terms of the percentage of students achieving the benchmark grades at GCSE but also in terms of the achievements of most students within successive cohorts, in terms of average points score. Equally significantly, perhaps, is the evidence offered by value-added data. Detailed analysis of students' GCSE performance against Yellis ${ }^{2}$ predictions for recent years shows that a

\footnotetext{
${ }^{2}$ YELLIS (Year 11 Information System) is a value-added monitoring system developed by the Curriculum Evaluation and Management Centre at the University of Durham. The YELLIS system provides a wide range of performance indicators for students aged 14-16, and makes possible comparisons of student achievement against their prior achievement, and against other students nationally by subject, student and school.
} 
considerable number of girls and boys perform significantly better at GCSE than predicted by Yellis data; in each year (Table 6.2), at least 50\% more boys and girls achieved the benchmark grades at GCSE than predicted by Yellis.

In achievement terms, the Partner schools had contrasting achievement profiles at the outset of the Project. Thus in school A, $25 \%$ of boys and $34 \%$ of girls achieved A*-C grades in 2001, at the outset of the Project's work with the school, reflecting the challenging nature of the catchment area with extensive multiple deprivation. A new headteacher had arrived in the previous year, inheriting a recent Ofsted report which had placed the school in the 'serious weakness' category. Partner school B, had higher levels of overall achievement, serving a catchment area which had more affluent areas within its areal mix, but a persistent gender gap similar to school A; in 2001, $49 \%$ of boys and $56 \%$ of girls achieved the benchmark grades.

Table 6.1 Achievement profile at GCSE, 1997 - 2004 (Originator School)

\begin{tabular}{|c|c|c|c|c|}
\hline & $\begin{array}{c}\text { Average } \\
\text { points score: } \\
\text { boys }\end{array}$ & $\begin{array}{c}\text { Average } \\
\text { points score: } \\
\text { girls }\end{array}$ & Boy/girl ratio & $\begin{array}{c}\text { Gender gap points score } \\
\text { (in girls' favour) }\end{array}$ \\
\hline 1997 & 33 & 34.3 & .96 & 1.3 \\
\hline 1998 & 31.3 & 35.0 & .89 & 3.7 \\
\hline 1999 & 37.3 & 40.8 & .91 & 3.5 \\
\hline 2000 & 42.8 & 43.2 & .99 & 0.4 \\
\hline 2001 & 43.4 & 45.9 & .95 & 2.5 \\
\hline 2002 & 42.5 & 46.3 & .92 & 3.8 \\
\hline 2003 & 36.7 & 46.4 & .79 & 9.7 \\
\hline 2004 & 50.7 & 48.1 & 1.05 & -2.6 \\
\hline
\end{tabular}

Table 6.2 GCSE performance against Yellis predictions, 2002 - 2004

\begin{tabular}{|c|c|c|c|c|c|c|}
\hline & $\begin{array}{c}\text { Yellis } \\
\text { prediction } \\
\% 5 \mathrm{~A}_{-} \mathrm{C} \\
\text { grades 2002 }\end{array}$ & $\begin{array}{c}\% 5 \mathrm{~A}_{-\mathrm{C}} \\
\text { grades 2002 }\end{array}$ & $\begin{array}{c}\text { Yellis } \\
\text { prediction } \\
\% 5 \mathrm{~A} \text {-C } \\
\text { grades 2003 }\end{array}$ & $\begin{array}{c}\% 5 \mathrm{~A}^{*}-\mathrm{C} \\
\text { grades 2003 }\end{array}$ & $\begin{array}{c}\text { Yellis } \\
\text { prediction \% } \\
5 \mathrm{~A}^{*} \mathrm{C} \\
\text { grades 2004 }\end{array}$ & $\begin{array}{c}\% 5 \mathrm{~A}_{-} \mathrm{C} \\
\text { grades 2004 }\end{array}$ \\
\hline boys & $33 \%$ & $60 \%$ & $31 \%$ & $61.5 \%$ & $50 \%$ & $74 \%$ \\
\hline girls & $40 \%$ & $68 \%$ & $43 \%$ & $79.5 \%$ & $52 \%$ & $81 \%$ \\
\hline
\end{tabular}

\section{Case Study 2}

\section{The background}

This triad consisted of three 11-18 inner-city comprehensive schools in the West Midlands. Two were virtually monocultural, serving well-established Asian communities, were boy dominated, and had $90 \%$ of students speaking English as an additional language. The third 
school served a more ethnically diverse but largely stable neighbourhood, with $33 \%$ of students speaking English as an additional language. The intake of all three schools was skewed towards the less able, with few very able students and the proportion of students with special educational needs was above the national average.

The Originator school had established a small-scale mentoring programme in KS4, targeting students who were underachieving, and this had contributed to a real improvement in students' results, as can be seen in Fig. 6.1.

Fig. 6.1: Achievement Profile at GCSE for the Originator School (\% students achieving 5(+) $A^{*}-C$ grades 1998-2004)

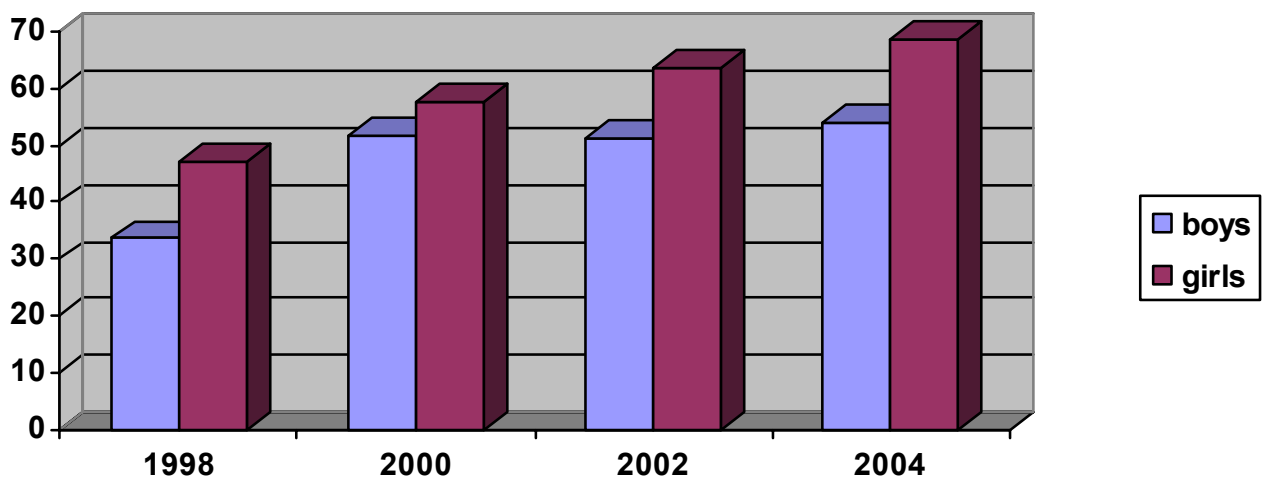

The Partner schools had limited prior experience of mentoring programmes, but saw the potential of such a scheme in key stage 3 , and consequently implemented a similar mentoring scheme (the Activate Programme) in Years 8 and 9. Three cohorts of boys were mentored as part of this programme. The results of these boys, comparing their actual results in the English, maths and science tests against projections based on CAT scores, which were taken on entry to secondary school, are given in Table 6.3. This analysis suggests that the attainment of $73 \%$ of boys in partner school A and $61 \%$ of boys in school B either matched or exceeded their predicted results. Given that all students participating in Activate were felt to be underachieving prior to their involvement in the programme, the fact that a reasonably high proportion of these students went on not only to attain at their projected level but in some cases to exceed their projections $(43 \%$ in school A, $32 \%$ in school B) suggested the programme had real potential to raise achievement.

Table 6.3: Breakdown of Activate Students' Key Stage 3 Results

\begin{tabular}{|c|c|c|c|c|c|c|}
\hline \multirow{2}{*}{ Attainment (no. of pupils) } & \multicolumn{3}{|c|}{ Partner School A } & \multicolumn{3}{c|}{ Partner School B } \\
\cline { 2 - 7 } & 2002 & 2003 & 2004 & 2002 & 2003 & 2004 \\
\hline Attainment below projection & 5 & 2 & 5 & 5 & 3 & 3 \\
\hline Attainment matches projection & 4 & 6 & 3 & 2 & 1 & 5 \\
\hline Attainment exceeds projection & 3 & 7 & 9 & 2 & 5 & 2 \\
\hline Total No. Students & 12 & 15 & 17 & 9 & 9 & 10 \\
\hline
\end{tabular}




\section{Target-Setting and Mentoring Approaches}

It is important to stress, as we make clear in chapter 3, that it is difficult with any intervention strategy, to disentangle the specific impact of any one strategy, and to make claims for one intervention in isolation from its context. We cannot be sure, in any school within any triad, that the effect of a particular intervention, in isolation, has been responsible for transforming attitudes, motivation and achievement. In some schools within these triads, for example, changes at senior management level (significantly in two schools, the appointment of new headteachers after periods of stability and / or stagnation) have led to revitalisation and periods of inspirational leadership; a renewed emphasis on learning and teaching strategies within the classroom and an allied focus on assessment for learning have raised expectations and possibilities; attempts to create an 'achievement for all' culture within the school has contributed to students' changing self-perceptions. Central to all these initiatives, however, has been the development of a vigorous and highly interventionist form of target-setting and mentoring.

The key question, though, is why and how target-setting and mentoring in some schools has been effective in contributing to these improvements in students' achievements, attitudes and expectations, whilst in other schools within the triads there has been far less impact.

\section{Students' Perspectives}

During the Raising Boys' Achievement Project, we have interviewed students, both boys and girls, individually and in single-sex focus groups, as they have progressed through their GCSE courses (in Case Study 1) and across key stage 3 (in Case Study 2). The voices of these boys offer revealing insights into the nature of mentoring, and highlight some of the key issues which schools must resolve if mentoring and target-setting are to be most effective. Throughout these interviews, there is an emphasis on easy communication and informality:

My mentor listens well, we have a good conversation, it's just like talking with your mates - well, perhaps not quite! He's a good mentor because he lets me talk to him.

You don't really realise how the relationship is building up. Your mentor talks to you as another adult, in here you're an individual person. It really boosts your confidence... you don't really see how it's helping till afterwards.

on establishing confidence and raising aspirations:

We can review our grades and see what can be done to make them better; it encourages us to keep going, and it motivates us. We'll definitely do better in GCSEs: our predictions have got better through the year. I'm certainly optimistic about what I'll get.

on achievable targets rooted in realistic expectations:

Seeing the grades is crucial: in my previous school mentoring wasn't linked to grades; it wasn't very precise or helpful. Here, you get to see where you're at and to know what to do to get higher... the targets are really important.

on practical support, clear advice and immediate resolution of issues: 
When I'm behind with something, I can really talk about it. My mentor will talk to the class teacher, and then to me, about how I can catch up; sometimes they'll set up extra sessions for a group of us.

I will tell the mentor stuff and we can work our way around a problem if I disagree with the teacher. The mentor and the teacher are in good communication. It helps to have your mentor, because he's talking for you, so it makes it easier to get the point across.

These insights enable us to confirm many of the qualities of 'good mentors', as perceived by students. In essence, it appears that 'good mentors':

- don't show irritation and have lots of patience;

- are willing to listen to students and are enthusiastic for them;

- enable students to establish a dialogue with them;

- convince students of their own ability and what they can achieve;

- are prepared to accept that a student is not perfect, and to look ahead and take a positive view on things, rather than simply 'going on and on if you've taken a dip';

- $\quad$ are straight talking, honest and genuine: 'he's dead canny and gets on so well with $u s^{\prime}$;

- 'give you advice and support, and strategies to help you learn, but give you responsibility because you have to go and do it, to take responsibility yourself for your time-management, and your own revision';

- realise there is life outside school!

There is a sense, in many of these interviews, of real understanding and valuing between mentor and student, with potentially disengaged boys and girls developing a sense of selfbelief, and being willing to accept praise openly when it is offered, appreciating that it is both genuine and realistic. The mentoring process is sustained, though, through regular and frequent meetings timetabled into the yearly calendar, with mentors working hard to protect time for mentoring, to give it the highest priority to ensure that appointments can be kept by both pupils and staff. This gives students the chance to work with their mentor on clearly defined targets over short time periods, and to sustain the momentum of learning and motivation. This was a vital element, particularly for some boys who welcomed this and saw it as offering regular feedback within a supportive context.

Another feature of these mentoring systems is the way in which the agenda is set, first and foremost, within an academic context, reflecting concerns with learning and with achievement, rather than simply with broader pastoral issues. Students are offered explicit strategies, too, which help them to minimise confrontations in the classroom and to manage their own anger. Students spoke of gaining more enjoyment from school, of being involved in less conflict with teachers and their peers, of noticing an impact on their own motivation and on how teachers in turn treated them:

I'm hardly ever sent out of lessons now. I used to spend as much time in the corridor as in the classroom! It's better now that someone is looking out for me, to help me. Now I'm a sensible person!

It is clear, too, from the students' responses, that mentoring is most effective when it makes reference to grade predictions which are both realistic and regularly updated: 
Having access to the grades is so important. It's given me more enthusiasm to work well because I can see what is possible. My grades sometimes surprise me. I've made a real move up. Seeing the grades and having encouragement and praise from my mentor gives me a real sense of achievement: you think you can do it after all. It's motivational ... you become convinced you really can do it!

At one level, then, the predominant tone of students' reactions suggest that this mentoring system is collaborative, supportive, persuasive in tone. There is indeed, a sense of negotiation between students and mentors, an acknowledgment that the mentoring scheme has the real interests and aspirations of the students at its core, that it is:

for us, not for the staff or the school. It really shows that they care for us as people, about what we can achieve and are capable of.

The process of triangulation, the discussion between student, mentor and subject teacher, is high profile in this approach, with students realising that mentors will intercede on their behalf, and that they will discuss alternative opportunities for them with their subject teachers. Students strongly welcome these interventions, and know that mentors will act with urgency to try to resolve a situation they have raised, even though it may not always be solved to their satisfaction! In our experiences working with these schools, this aspect gives mentoring strong credibility with students, establishes a degree of mutual confidence in the scheme, and enables a sense of reciprocity to be established. Students have a strong belief and confidence in their mentor, convinced that the mentors are working with and for them, 'battling' for them, in the memorable words of a Year 8 girl in the West Midlands, 'when no one else, at school or at home, seems to care'. Where this negotiation is delayed or ineffective, the scheme can quickly lose credibility with those very students it needs to hold.

Not all the schools in these triads have been able, as yet, to implement successfully this aspect of mentoring, and it is clear that there is continuing unease in some schools, where the negotiation between mentor and student is seen as having the potential to create a feeling of complicity which might undermine subject teachers. It is equally clear, however, that mentoring is most effective where such open negotiation between mentor and subject teacher has been established, and where it is transparent to the student.

\section{Assertive Mentoring}

There is a contrasting aspect of these mentoring schemes, however, which is equally crucial in determining their effectiveness, and which we describe as assertive mentoring. Although much of the mentoring is supportive, positive and relatively relaxed in tone, there is frequently a change in emphasis as the Year 10-11 cycle progresses, with more direct and challenging interactions with students. The following conversation, between a mentor and a female student successfully persuades the girl to focus efforts on the subject rather than the teacher:

S: 'I'm not bothered if I pass or not ... I just want to get out of his class because he's pushing me too hard'

T. 'But who will suffer then, the teacher or you?'

S. 'Who cares ... I don't want to work for him!' 
T. 'But you've done loads of work already; whose benefit is that for? It's your qualification which is going to be the loser if you go off in a huff, not the teacher, he won't lose.'

This change of tone is partly expressed in the rigour and persistence with which mentors pursue issues and targets raised in previous sessions, exploring which aspects of targets might not have been met, but mentoring sessions can also be characterised by challenge and the demand for more commitment from the student. In these instances, usually in mentoring sessions with specific under-performing students (mostly but not always boys), this embodies a more direct interventionist style of mentoring, offering strategies and negotiation but making assertive demands in return:

'I'll talk to your (subject) teacher so that you have opportunity to redo that piece of work

... You got a 5 in your SATs in year 9 ... there's no way you're going to get a D at GCSE ... you might as well have sat in the yard instead of going to (subject) lessons these last two years ... I'll have it sorted by Monday .... The problem, though, is that I haven't seen you around enough in lunchtime clubs and after school clubs ... Mr D is staying behind after school, are you? ... So more effort is required, my son ... see to it ... please ... OK?'

This is a more direct style of mentoring, relating partly to the personality of the mentor, but also to the implicit agenda brought to the sessions by the students, and to the timing of the mentoring sessions (March in Year 11) within the GCSE cycle. There is an attempt to establish the notion that the students have a personal responsibility not only to themselves, but to honour the commitment they are making to their mentor and to their subject teachers. There is still informality and charisma to establish rapport, but within this, there is also a direct and forceful style, with an emphasis on reciprocity, on keeping 'the bargain' made earlier in the year. Mentors know their students well, enabling them to refer back to their personal and family circumstances and to issues of illness and attendance, but it is also clear where the power is located, with mentors assertive and initiating the direction of the dialogue, focusing straight in on problem areas and subjects, establishing rapport and offering praise, but also challenging:

Your teacher of 20 years experience tells me you're on course for an E grade in this subject, so whether you think you're going to get a B grade is irrelevant! Her experience, and mine, is telling you that unless you change your attitude, approach, work rate, you're in for a shock. It's no B you're looking at, trust me on that one!

It is worth reiterating here, though, that there is a further aspect of the mentoring carried out in these contexts. We see this confrontational challenging aspect of mentoring as addressing directly issues of laddishness and macho image; the mentor in such a context provides some boys (and the occasional girl) with a way not to opt out. The intervention of senior staff, who are seen to be monitoring these students tightly, gives them a justification to use to their peers, to enable them to continue to work, to increase their involvement, to work to meet achieve targets. The school, through its strong intervention at this stage, is offering them almost a face-saving device to enable them to work without undermining their own sense of being a 'lad' or a 'ladette'.

\section{Pre-conditions for Successful Mentoring and Target-setting}

In some schools within these two triads, mentoring has been a crucial strategy in helping to raise the achievement levels of both boys and girls. In other schools, where key elements 
of the scheme have been embraced less enthusiastically, it has been less transformational. If the effectiveness of mentoring and target-setting is to be maximised, we suggest that it needs to embody a number of essential characteristics:

\section{To be based upon precise and detailed monitoring of students' progress}

Some schools have traditionally 'under-graded' students at key points, to 'shock' them and attempt to increase motivation. In other schools, teachers have sometimes 'over-graded' to boost confidence and to give reassurance. Both approaches can totally invalidate mentoring and reporting since there is no secure foundation for the grades and for subsequent target-setting. Equally, the use of historic data, without contextualisation in terms of value-added measures and intake scores, can sometimes reinforce historic underachievement, and aspirations which are too complacent; in such contexts, underachievement is sometimes widespread.

We believe that target-setting can make a powerful contribution to improving students' achievements, and that the definition of realistic grades, regularly reported and contextualised within value-added data, has been crucially important because it has enabled teachers (as well as students) to raise their own expectations and aspirations.

The use of value-added data to inform teachers and students of realistic possibilities thus not only avoids the dangers of an over-cautious approach to predictions which can demotivate and in turn contribute to the self-fulfilling prophecy of lower achievement, but can also reassure and build confidence. The importance of this cannot be over-estimated:

We have a target-setting system in place which is remarkably efficient, accurate and effective. It is immensely comforting to us, and for our students, to be able to say, 'Don't worry: you continue working as you are, and we can predict with some assurance what you will achieve. It's particularly reassuring to girls, in that it reduces the stress, but it also builds up the confidence of boys: it makes them more assured, visibly more confident.

\section{To protect time for professional dialogue}

To establish a culture where there is such confidence in performance data takes understanding, commitment to maximising students' achievements, a willingness to engage with potential data and become data literate. To be effective in such a context, data analysis and target-setting have to be perceived as a core activity by all staff, and protected time identified for this activity. Our work with triad schools suggests that it is essential that teachers have defined time regularly to work in departments in such target-setting activities. This seems to us to be an important aspect of target-setting. In some schools, we are aware that students' targets have been fixed by senior staff, using prior attainment data and informed by national data and expectations, rather than leaving target-setting to subject departments where expectations are perceived as being too low. This may be an interim step necessary in some schools, but our experiences suggest that it is better to identify the time and space to stimulate professional dialogue and to challenge such perceptions of what is possible. In some schools, it is clear that this process of data analysis and target-setting has sharpened the thinking of some teachers about possibilities and outcomes, about what it really takes, on the basis of past evidence within the school, for students to be awarded a particular grade. As this increased realism has informed some 
teachers' thinking, so this has led to higher expectations of students and higher predictions by staff, and subsequently generated more assurance and confidence in students.

\section{To involve senior staff in mentoring}

Where mentoring is meaningful and effective, it is a high intensity activity involving an ongoing and regular dialogue with students. But it also involves communication and feedback with subject teachers, receiving 'alert forms' and discussing grade changes so that these can be rationalised to students, as well as requiring a response to issues raised by both teachers and students. If mentors are to negotiate effectively with other members of staff, to intercede on behalf of students, on the one hand, and to challenge students on the other, they need some seniority in the school, to be credible with other staff as well as students.

\section{To identify the rationale for mentoring and offer mutual support}

Central to the success of these mentoring schemes is the extent to which they develop the conviction in students that they are capable of achieving well, raising expectations where traditionally neither home nor community have high expectations of education and schooling, to motivate and sustain motivation. A tightly targeted mentoring scheme must overcome any feeling of stigma, and must be sensitively implemented to avoid conflicts with the laddish culture and macho images which some boys cultivate. The balance between collaborative mentoring and assertive mentoring is crucial here, in generating a sense of involvement and understanding in the participants and in establishing a sense of group identify with the aims and aspirations of the scheme:

It was good having some of my mates on Activate. It meant that several of us were trying to change, not just one, so we could help each other and ignore the jibes. I wasn't a lone ranger, so that helped a lot.

\section{Responsibility and intervention}

One of the most vigorous debates which has taken place across schools within these triads has revolved around the philosophy underpinning mentoring approaches. In one school, emphasis has been placed on a collaborative tone throughout, keen to develop students as independent learners well-equipped for the future, anxious not to generate antagonisms with borderline and disengaged students by creating pressures and tensions. Here it is made clear to students that they must want to achieve, must participate fully as proactive partners in mentoring and in learning, must identify willingly and co-operatively with the aims and aspirations of the school.

There is evidence from other schools that some students appreciate mentors who offer extrinsic motivation in 'forcing the pace to ensure they work', that they respond best to mentors who establish rapport with them but also make explicit and challenging demands. In some ways, too, the very students who benefit the most from mentoring are those who need more assertive guidance from mentors they respect, who need mentors to provide a context in which they are enabled to work and at the same time to sustain their own image and credibility.

Whilst this balance between responsibility and intervention can only be resolved in each school context, our own experience suggests that the most effective mentoring is that 
which creates a rationale which such students can use to their peers, to justify their involvement in academic work and offer as a reason for working, which enables them to address issues of image and group credibility.

\section{An Overview}

A small follow-up study among students who had progressed to sixth-form study from the Originator school in Case Study 1, showed that the mentoring they had received had been particularly appreciated by male students. A year later all six year 12 boys interviewed were able to identify the positive aspects of mentoring as: a general check on progress, encouragement to keep going, having someone there to help with any problems, giving advice on revision strategies and timing, as well as study skills and concentration, and direct advice on how to achieve better grades. Two talked about their mentors as friends, and some felt that the relationship had made a real difference to what they had been able to achieve:

\section{A hugely encouraging presence during year 11.}

Mr J really helped - he gave me an outlook in terms of grades and what might be. The sessions made me more aware and boosted me up - they kept me on target so I knew where I needed to do and what I needed to do to get further. He helped me get my head in order! If I didn't have the mentoring I wouldn't he here at sixth form: it changed my perspective of grades and what I could achieve - it really did change what I thought I could do.

Where target-setting and mentoring have been successful in transforming the achievements and attitudes of students, there appears to have been a crucial focus on the individual, on gaining and acting upon detailed knowledge of the potential of individual students, and conveying a sense of what might be possible. Target-setting and mentoring have the potential to be transformative on achievements at school, on attitudes towards schooling, on attendance at school and on behaviour whilst at school, when they possess certain characteristics and when certain pre-conditions have been met. In the light of our work with these triads, we suggest that target-setting and mentoring can be particularly effective with potentially disengaged boys and the fewer disengaged girls when those students:

- understand and 'buy into' the reasons for target-setting, when they feel sufficient autonomy to be in control of their own learning profile, and develop the skills and attributes of independent learners;

- perceive the tone of the scheme as supportive rather than oppressive

- understand that potential data create realistic expectations of what is possible, and gain a sense of self-esteem and confidence as learners when they realise what is indeed possible, given the historic trends within the school;

- are encouraged to make a comparison between their past self, present self and their aspirations for their future self as learners;

- appreciate that they are offered choice by their mentor, and are (made) aware of the responsibility conferred by choice;

- are offered a context where boys (in particular) can be offered an escape from the needs to confirm to a laddish, macho image, by the challenge and demands made by the mentor. 
In such contexts, successful target-setting and mentoring has helped to sustain not only students' sense of membership of school and their sense of self-esteem and self-worth, but their sense of agency, of having some voice and power to impact on their own contexts and lives, to make decisions and effective choices about their own futures. 


\section{CHAPTER 7: Organisational Contexts - Equal Opportunities in the Single-Sex Classroom}

\section{Single-Sex Classes in Mixed Comprehensive Schools : the issues}

One organisational aspect of schooling which has become high profile in the last decade has been the concern with single-sex classes for boys (and obviously then for girls!) in a number of co-educational schools in England. Sukhnandan et al (2000) reported that some teachers perceived single-sex classes as offering a number of advantages for the teaching of boys:

- the opportunity to use a variety of teaching strategies which were targeted to boys' needs and interests

- provision of a context in which teachers could challenge boys' stereotypes more effectively

- the existence of an all-male environment which was more conducive to learning, with fewer distractions and less embarrassment, enabling boys to be more open and responsive in class, and able to concentrate and participate more (see also Swan, 1998).

A different perspective suggests that single-sex classes reinforce rather than challenge sexrole stereotypes, and suggests that male teachers and boys welcome single-sex classes because they enjoy the 'boys-own' atmosphere of the classes and the opportunity for male bravado and bonding in such classes (Jackson, 1999). Such classes are seen as having the potential to reward macho-behaviour, on the part both of boys and male teachers, to allow more extreme and sexist language to pass unchallenged, to give new opportunities for oldstyle masculinity and to reinforce sexual stereotypes (Kenway et al., 1998).

This is not a new debate in one sense, of course, since single-sex classes and indeed, single-sex schooling, have been advanced in the past as one way of pursuing more equality of opportunity for girls. What is new is the advocacy of such an approach to support boys' education, although the debate has been resurrected despite the marked lack of evidence (notwithstanding Sukhnandan et al., 2000, and our own in-depth case study - Warrington \& Younger, 2001, Younger \& Warrington 2002) about the advantages of such schooling or teaching for boys.

\section{Single-Sex Classes within the RBA Project: initial work}

Our starting point on this aspect of our work was to conduct a review of single-sex teaching in co-educational comprehensive schools in England. This review, carried out by telephone survey across thirty-one schools, revealed a confused scene. In short, schools appeared to have implemented single-sex classes in an ad hoc way, for short time periods, frequently with little preparation and without consideration of the advantages which the distinctiveness of context might bring (Warrington \& Younger, 2003). The initiative was sometimes in place only for a half-term for one subject - so that making any realistic assessment of the effects on achievement was meaningless. Some schools believed it had raised achievement levels, while others did not; some had seen behaviour improve, while in others it had worsened; in some schools, girls were blossoming away from the distractions and the implicit need to help sustain boys' learning; in others girls and boys 
resented the single-sex context. In some schools, single-sex teaching was abandoned as abruptly as it was introduced, before a sustained time period had elapsed, and without evaluation of its benefits or disadvantages. In such contexts, it is not surprising that it was difficult to come to any clear conclusions about the effects on examination and test results, not least because of the diversity of subjects, year groups, sets and length of time the strategy was used.

\section{A Case Study}

This initial work did reveal, however, that there were a few schools where a more coherent and systematic approach to single-sex classes had been implemented, and where there was a conviction amongst key staff that this mode of organisation was contributing positively to improving achievement levels of both boys and girls. The focus of research in one triad, then, was to contribute to the debate about the potential of single-sex classes for boys' and girls' learning, and to consider under what circumstances, if any, such classes might offer better opportunities for boys and girls. In so doing, we have attempted to identify the essence of single-sex teaching in a particularly successful co-educational comprehensive school serving a socially diverse white population in southern England, and to support the transfer of this approach to two schools serving similar socio-economic contexts in Eastern England.

In the Originator school, single-sex teaching was one of a number of organisational strategies which aimed to improve the achievement levels of boys and girls within the context of establishing an achievement culture within the school, with high aspirations for and expectations of all students. Initially tightly targeted at boys and girls who were perceived as being in danger of under-achieving in English at GCSE, single-sex teaching was subsequently expanded and introduced with middle ability sets in Mathematics, Science and Modern Languages.

Both Partner schools had some prior experience of involvement with single-sex teaching in key stage 4, but the philosophy underpinning single-sex teaching, and the associated teaching strategies were less developed than in the Originator school. The rationale behind the introduction of single-sex teaching also differed in the two Partner schools; in school $\mathrm{B}$, it focused on improving the achievement of boys in English, whereas in school C, the strategy was initially linked directly to the perceived under-achievement of middle set girls in Mathematics.

In the Originator school, single-sex teaching has been one of the factors which has helped to transform achievement. Not only have boys 'out-performed' girls in particular years, but the performance levels of both girls and boys, year-on-year, have generally followed an upward trajectory over the eight years analysed (Table 7.1).

In more specific terms, both boys and girls in single-sex classes for English in 2002-03 recorded a higher points score in the subject than they obtained overall, although this pattern was not replicated in Maths (Table 7.2); equally, boys' achievements in this school in French were sustained at a level comparable to their average performances in other subjects, and did not follow the 'usual' under-performing boys' pattern in modern languages. 
Table 7.1: GCSE: average points score /\% student intake achieving 5(+) $A{ }^{*} C$

\begin{tabular}{|c|c|c|c|c|c|c|c|}
\hline & \multicolumn{4}{|c|}{ GCSE average points score } & \multicolumn{3}{c|}{$\%$ intake $5(+)$ A*-C grades } \\
\hline School A & boys & girls & boy/girl ratio & gender gap & boys & girls & gender gap \\
\hline 97 & 47.3 & 48.8 & 97 & 1.5 & 68 & 68 & 0 \\
\hline 98 & 47.9 & 50.5 & 95 & 2.6 & 69 & 73 & 4 \\
\hline 99 & 55.3 & 53.3 & 1.04 & -2.0 & 83 & 79 & -4 \\
\hline 00 & 52.3 & 54.5 & 96 & 2.2 & 79 & 81 & 2 \\
\hline 01 & 54.6 & 58.7 & 93 & 4.1 & 73 & 82 & 9 \\
\hline 02 & 58.1 & 59.2 & 98 & 1.1 & 82 & 79 & -3 \\
\hline 03 & 55.6 & 60.0 & 93 & 4.4 & 75 & 83 & 8 \\
\hline 04 & 56.3 & 61.5 & 92 & 5.2 & 81 & 82 & 1 \\
\hline
\end{tabular}

Table 7.2 : Attainment of boys and girls in single-sex classes

\begin{tabular}{|c|c|c|c|c|c|}
\hline & $\begin{array}{l}\text { \% gaining C } \\
(+) \text { in } \\
\text { subject }\end{array}$ & $\begin{array}{c}\% \text { gaining } \\
5(+) \mathrm{A} *-\mathrm{C} \\
\text { grades overall }\end{array}$ & $\begin{array}{c}\text { Average } \\
\text { points score } \\
\text { overall } \\
\text { subjects }\end{array}$ & $\begin{array}{l}\text { Points score } \\
\text { in subject }\end{array}$ & $\begin{array}{l}\text { \% gaining } \\
\text { higher score } \\
\text { in subject } \\
\text { than their } \\
\text { overall } \\
\text { average }\end{array}$ \\
\hline $\begin{array}{l}\text { Boys in } \\
\text { English } \\
\text { language }\end{array}$ & 77 & \multirow[t]{2}{*}{81} & \multirow[t]{2}{*}{4.8} & 5.4 & 67.7 \\
\hline $\begin{array}{l}\text { Boys in } \\
\text { English } \\
\text { literature }\end{array}$ & 87 & & & 5.8 & 90.3 \\
\hline $\begin{array}{l}\text { Girls in English } \\
\text { language }\end{array}$ & 80 & \multirow[b]{2}{*}{83} & \multirow[b]{2}{*}{4.8} & 5.2 & 63.3 \\
\hline $\begin{array}{l}\text { Girls in English } \\
\text { literature }\end{array}$ & 97 & & & 5.5 & 80.0 \\
\hline Boys in Maths & 59 & 83 & 5.0 & 4.8 & 27.6 \\
\hline Girls in Maths & 48 & 90 & 4.8 & 4.4 & 22.6 \\
\hline Boys in French & 81 & 88 & 5.4 & 5.3 & 44.0 \\
\hline Girls in French & 78 & 89 & 4.7 & 4.7 & 33.3 \\
\hline
\end{tabular}

The performance data through time in the Partner schools is less clearcut, however. In both schools, all the boys interviewed were predicted to be on the C/D GCSE borderline in the specific subject, in either English or Maths, when their GCSE course began, and their ongoing reports during the GCSE years suggested a fluctuating level of performance, such that - at the time of their last interview in May 2003 - neither the boys themselves, nor 
their teachers, were confident that they would achieve a higher level GCSE pass (at grade C).

Nonetheless, in school B, of the students taught in single-sex classes, all girls in school B passed both English Language and English Literature in 2003, compared with $81 \%$ of boys for English Language and 56\% in English Literature. Interestingly, however, the boys' average points score for English (4.9) was higher than that for other subjects (4.5). In school C, although both boys and girls in single-sex classes performed better in Mathematics than might have been predicted from an analysis of their cognitive ability scores, with typically over $50 \%$ of both boys and girls exceeding predictions in any year, this pattern was also apparent from the results of boys and girls, drawn from a similar population in the other half of the year group, and taught in mixed classes for mathematics.

Any analysis based simply on performance data must be tentative, therefore, because of sample size and the difficulty of isolating the impact of being taught in single-sex classes from other factors, but there is some limited evidence here - from both the Originator school and one of the Partners - of positive impacts of single-sex classes on achievement levels.

\section{Learning in Single-Sex Classes: the Students' Perspective}

Previous studies have suggested that students feel that single-sex teaching has a positive impact on their learning and motivation (Barton, 2000; Jackson \& Smith, 2000; Younger \& Warrington, 2002), although some studies have shown that boys in particular can feel hostile and resentful of single-sex classes (Kenway et al, 1998); Jackson, 2002; Warrington \& Younger, 2003).

In this case study, a questionnaire completed by 163 girls and 165 boys across all three schools showed some equivocation and ambivalence in students' responses across schools, with girls on the whole responding more positively than boys (Table 7.3). More girls in all three schools, and boys in the Originator school and Partner school B, agreed that singlesex classes aided their concentration and helped them to feel more confident about their work, and in each school, more girls and boys felt that there was a better atmosphere in single-sex classes. There was less consensus along gender lines on other questions, however. More girls in all three schools felt more willing to answer questions in single-sex classes than in mixed classes, and felt that the single-sex teaching mode was a good idea which ought to be continued in these subjects. Conversely most girls in each school agreed that they did not want single-sex classes extended to other subjects, did not feel it was easier to accept praise from the teacher in a single-sex class than in a mixed class, and did not agree that teachers taught differently in such classes. Boys generally were more negative in their responses, particular in responding to questions about better behaviour, accepting praise and willingness to answer questions, but what is equally interesting is the differential responses of boys across the three schools.

There were generally more positive responses across the range of questions from boys in the Originator school than in Partner school B, while boys in Partner school C reacted negatively to virtually every question. This differentiation in responses may be due to the subject taught in single-sex classes in school $\mathrm{C}$, and the possibility that boys did not see themselves as needing any special support or difference of approach in mathematics. In the other two schools, boys may well have responded more positively because they saw the 
mode of organisation as supporting their learning in subjects, such as English and French, in which they perceived themselves to be weaker. Equally, there may be issues to do with transferability and sustainability, with the ethos and clarity of purpose to support singlesex teaching more in place in the Originator than in either Partner school.

Table 7.3: Questionnaires responses from students in single-sex classes, 200203: (65 girl / 84 boys in school A, 68 girls / 62 boys school B, 30 girls / 19 boys school C)

\begin{tabular}{|c|c|c|c|c|c|c|}
\hline \multirow[b]{3}{*}{ School } & \multicolumn{6}{|c|}{$\%$ students agreeing / strongly agreeing with statement } \\
\hline & \multicolumn{3}{|c|}{ girls } & \multicolumn{3}{|c|}{ boys } \\
\hline & A & B & $\mathrm{C}$ & A & $\mathrm{B}$ & $\mathrm{C}$ \\
\hline $\begin{array}{l}\text { I have usually concentrated better } \\
\text { in single-sex classes }\end{array}$ & 73 & 63 & 83 & 62 & 63 & 16 \\
\hline $\begin{array}{l}\text { I have felt more confident in } \\
\text { single-sex classes }\end{array}$ & 78 & 65 & 90 & 57 & 73 & 32 \\
\hline $\begin{array}{l}\text { I have usually been better } \\
\text { behaved in single-sex classes }\end{array}$ & 61 & 39 & 63 & 46 & 45 & 48 \\
\hline $\begin{array}{l}\text { I have usually produced better } \\
\text { work when I am taught in a } \\
\text { single-sex class }\end{array}$ & 64 & 42 & 63 & 54 & 57 & 26 \\
\hline $\begin{array}{l}\text { I have usually felt more willing to } \\
\text { answer questions in single-sex } \\
\text { classes }\end{array}$ & 70 & 56 & 57 & 46 & 53 & 42 \\
\hline $\begin{array}{l}\text { In single-sex classes, I think it has } \\
\text { been easier to accept praise from } \\
\text { teachers for my work }\end{array}$ & 39 & 39 & 40 & 54 & 21 & 20 \\
\hline $\begin{array}{l}\text { I think being taught in single-sex } \\
\text { classes is a good idea, and should } \\
\text { be continued in these subjects }\end{array}$ & 73 & 66 & 73 & 62 & 50 & 26 \\
\hline $\begin{array}{l}\text { I think single-sex classes should } \\
\text { be extended to other subjects }\end{array}$ & 44 & 25 & 50 & 40 & 21 & 16 \\
\hline $\begin{array}{l}\text { There is a better atmosphere in } \\
\text { single-sex classes }\end{array}$ & 85 & 76 & 70 & 85 & 71 & 84 \\
\hline $\begin{array}{l}\text { Teachers teach differently in } \\
\text { single-sex classes }\end{array}$ & 48 & 24 & 30 & 44 & 18 & 11 \\
\hline
\end{tabular}

When asked to expand on their answers, and identify the two best things about being taught in single-sex classes, girls and boys both said that it was easier to concentrate because there were fewer distractions from the opposite sex. To girls, these distractions might be from 'the noise generated by immature boys' to the fact that 'we don't have to worry about what we look like if boys are not there'. To boys, it was easier to concentrate when there were no temptations to look at or talk with girls, since in the memorable words 
of one boy, 'my hormones are not dancing to the beat of the night!' Both girls and boys welcomed the removal of social pressures, to perform to an image to sustain femininity or laddishness:

you don't need to act as though you're really cool, especially when you're not feeling as though you are! (Y11 girl)

Students also felt able to participate more readily, to offer answers and ask questions and to offer opinions, because they were surrounded by friends so they were less intimidated:

You feel braver and less embarrassed in offering answers, because there are no boys to make fun of you when you are wrong.

It gives you a lot more confidence to answer questions in class because there is not so much pressure and embarrassment if you are wrong as there would be with girls about.

Girls and boys wrote of more informal relationships in single-sex classrooms, with teachers tolerating a more relaxed and friendly attitude, being less stressed because there was less off-task behaviour and more willing to talk about things they knew would interest classes. Girls, in particular, felt that they learned better because the teachers could teach more, with less need to be continually disciplining disruptive boys; boys felt that they received more teaching and more attention from their teachers because girls were not there to dominate the questioning.

Boys in particular, claimed that single-sex classes in English allowed them more freedom to work harder without worrying about stereotypical expectations and their own image, particularly that they were not supposed to enjoy English. They spoke of being able to talk about feelings and express opinions about books and poetry, to target coursework without feeling intimidated by girls, and to study and enjoy the romantic texts:

We don't just do war poems and Macbeth, we do Wordsworth too. It's a challenge, in a way, which MrJ sets us to show the girls we're capable of doing it, but I couldn't talk about these things if there were girls there!

There's more participation in the lesson because he involves all of us, and no one is shy or afraid to express an opinion ... you know other boys won't laugh at you and you don't lose face.

Equally, girls spoke of it being 'far less embarrassing when doing talks if the blokes aren't there to rubbish you', of 'being able to say what you really feel about plays and emotional stuff and things like that' and of there being 'no pressure to be loud and funny in front of the guys'. Boys, too, acknowledged 'feeling more confident, especially when giving presentations and asking questions', of 'not being nervous and messing up so much when talking about the work' and of being 'not afraid to talk about feelings in poetry and plays'.

More students in the Originator school A and in Partner school B, then, and girls in Partner school C, suggested that single-sex classes can be developed to support the learning of girls as well as boys, rather than discriminating against or undermining girls' achievements. Some questions remain, however, particularly a common worry about issues of classroom management and the extent to which all-boys' classes were more challenging for teachers. This was certainly the perception amongst some teachers in the three schools, and the concern that aggressively macho behaviour was exacerbated simply through a concentration of numbers, with a subsequent worsening of boys' behaviour. 
There is nonetheless a danger here of assuming that a reversion to mixed classes may 'solve' the issue; indeed, in some cases, such a change simply means that girls are made to bear the brunt of the failure of the school to develop an effective approach to classroom management and communication.

A further factor is the suggestion that single-sex classes may initiate bonding between male teachers and boys, reinforce laddishness and create images which some other boys find difficult to identify with. This is not an easy issue to pursue, particularly when we have met individual boys only on a limited number of occasions over a two-year time span, but our interviews with 'non-macho' boys suggest that these boys did not feel exposed in single-sex classes. Whilst, at one level, there were hints of more banter and occasional rowdiness in all-boys' lessons, the occasional sexist comment at girls' expense, and discussion about football, such boys told us - without exception - that they felt at ease and comfortable, that they did not experience bullying or aggressive behaviour from other boys, and that they were not intimidated by the atmosphere in all-boys' classes:

The teacher is a man's man, I suppose, he talks about football to some of the boys. I'm not into football, but it's $O K$... It's just how it is, because it's only a starter like, for a few minutes ... the atmosphere is so much more relaxed and joyful without girls, and he's such a good teacher ... it's great being in the lesson!

There are obvious dangers here, as we indicate earlier, of the creation and perpetuation of stereotypical macho environments. Whilst there was little evidence in this case study to suggest that such stereotypes were being created, it is an issue to which all teachers must be alert when teaching in single-sex contexts.

\section{Teaching single-sex classes}

Earlier work has suggested that there is little evidence that most teachers modify their teaching style and strategies to meet the perceived differing needs of boys and girls, and plan different lessons for boys and girls (Sukhnandan et al, 2000; Younger \& Warrington, 2002). There is a dilemma here which we have wrestled with throughout the Project: are single-sex classes to be advocated because it is possible to create a different ethos and atmosphere for learning, or should they explicitly recognise that boys and girls do respond differently, in certain contexts, to different teaching-learning styles?

Classroom observations and ongoing discussions with teachers in each school suggested that the nature of good practice teaching in single-sex contexts could be clearly identified and exemplified. In the Originator school, these characteristics had been defined, discussed within staff groups, trialled and subsequently refined. Inter-school discussions across the triad suggested there was a clear and widespread acceptance of the main issues involved in good practice, a clear sense of the issues and challenges which such classes presented, and a determination to devise and articulate strategies which could be implemented by teachers. Although, in the first instance, these approaches were developed with the challenges of teaching all-boys' classes very much in mind, as the research developed, it became evident that such classroom strategies were equally applicable to all-girls' classes. Indeed, these pedagogical practices were highly effective in gaining student involvement and in enhancing motivation regardless of whether the class consisted of boys or girls. Identified good practice, therefore, emphasised issues such as timing, pace and variety, the need for teacher presence and for high expectations. Crucially, though, there emerged a 
focus on the need for teaching and learning to take place within a set of tightly defined parameters, and within a context almost of persuasion and identification with the students (Fig 7.1).

\section{Fig 7.1 Single Sex Teaching: Good Practice in the Classroom}

\section{(adapted from Staff Development material in Originator School)}

- A clear and sharp beginning, within a coherent structure which is clearly explained to class; the 'point' of doing the activity must be always explained

- Vibrant and fast teacher - pupil interactions

- High levels of teacher input

- The use of a proactive and assertive approach, which does NOT become negative or confrontational

- Constant reinforcement of high expectations of the boys / girls

- Establishment of absolute 'base line' rules which, when broken, incur known and consistent sanctions

- Active promotion of a team ethic, to forge an identity for the class of which the students can feel part

- Short term targets, with several different activities taking place within each lesson, to tight and agreed time limits

- The use of public praise when the teacher knows that this can be received and acknowledged by individuals

- The use of humour, informality and discussion of topics with which the students identify (fashion, sport, music, technology), to consolidate rapport

.... but also remember ....

- That students are individuals ... we need to know them as individuals

- More sensitive students can be intimidated by the atmosphere in single-sex class and become reluctant to contribute ... they might need space to work as individuals, to express themselves differently

- However experienced the staff, sexist repartee (whether with boys or girls) needs to be strongly discouraged, because although it may help teachers on occasions to establish rapport and togetherness with students, it can quickly escalate and degenerate, and create counter-productive images and stereotypes.

- Sexist comments or behaviour, and stereotypes must be challenged, not condoned.

There is more sophistication here, though, than might be at first apparent. Successful teaching and learning in single-sex contexts demanded not only such pedagogic practices in the classroom, but these teaching strategies needed to be situated within a set of beliefs, attitudes and expectations held by teachers, and strongly supported by senior managers 
within each school. Classroom observations tended to confirm that the most successful teachers were those who generated a sense of belief and mutual respect amongst the students and who offered positive and immediate feedback. Such teachers were prepared to engage with the class in accepting irony and humour, and acknowledged where the students were coming from, but established a context of clear expectations which were understood by all.

At times, of course, this is problematic, and can lead to a situation where teachers identify with and encourage laddishness, where stereotypes are reinforced, where female teachers almost 'flirt' with boys in a desperate attempt to establish rapport. We would strongly disapprove of such a situation. This need not be the case, however, for in other contexts, this approach can establish remarkably successful contexts for learning, regardless of the gender or age of the teacher.

You need to be able to relate well to challenging boys, boys who in other contexts might be seen as unteachable, to have high expectations of them, in terms of behaviour, work, their relationship with each other ... to be very, very positive with them, to be upbeat, informal, smiling. I let them know I believe in them, that I'm committed to them and to their learning. They know I'm fighting their corner: once I've got that relationship in place, you don't have this roller-coaster of negativity, of disgruntlement. (female English teacher, school B)

In similar vein, a male English teacher in school A talked of the need to:

try to engender a collaborative yet competitive atmosphere, so that they feel comfortable in front of each other. I allow them a little more leeway in terms of what they talk about, but the essence of the relationship is mutual respect, so that when I say 'that's enough', it will be effective!' Also, I give a lot of praise in ways which I think they feel to be acceptable, I'm very careful in who and how I praise. I encourage them to feedback on each other's work, generating a sense of a team, because all of them - however much trouble they're in elsewhere - perceive GCSEs as important and they all want to do well. So they're not going to stitch up their mates by misbehaving. It's almost as though there's the rules of the playground going on in the class, they tell each other if they think it's out of order and I foster that!

This teacher was prepared to accept irony and humour directed at him by specific students, responding to spontaneous comments offered during the course of a lesson, acknowledging 'where they are coming from', but within a context of clear expectations which were understood by all.

Both here, and in the case of female Maths teachers in school C, the teachers clearly showed that they were accessible to their students, supportive and engaged positively with their learning, encouraging groupwork and work-related exploratory talk. Faced with assertive behaviour within their classes, the teachers used a variety of techniques (occasional gentle humour, a smiling informality of approach and praise) to ensure that learning took place against a variable backcloth of noise and jocularity. The essential tone of these classrooms was one of mutual respect, a shared understanding that learning could at times be sustained within a informal, relaxed context, but equally an understanding held by virtually all the students that the teachers were setting the tone and maintaining the ethos of the lessons. Such classes could and were brought sharply to order when it was judged to be necessary, by teachers who were aware that it was their responsibility not to cede power or authority to the students within their classes. 
Confirmation of the effectiveness of this approach was seen in the classroom. Teaching a group of Year 11 boys who were restless and on the edge last period in an afternoon, a female modern languages teacher accepted jovial irreverent responses, but used them and skilfully turned them back on pupils, to engage them. Tired, distracted, a trifle disengaged and offering somewhat challenging 'in your face' behaviour, the boys nonetheless responded positively to the very sensitive handling they received, acknowledging the clear, firm line the teacher had taken, and welcoming the tone of her response. It was as if the boys needed to have the context spelt out for them all, so that no one would lose face if they behaved, so that all had a reason they could use to justify their behaviour to each other.

It is possible, then, to define strategies which might be effective in the classroom in teaching boys'-only classes, to identify the essence of good practice. But we have also seen such strategies fail with some classes, where teachers have failed to recognise the dangers of the intimidation of 'non-macho' boys or forgotten that laddishness in such classes needs to be kept in check rather than encouraged. We return to the point about the crucial importance of teachers' beliefs, attitudes and expectations, of their commitment to the students they are teaching, and for the need for senior managers to support these teachers publicly and proactively. Boys-only classes are frequently more challenging, and teachers need a set of carefully devised strategies, supported by positive beliefs and expectations, if they are to succeed in such contexts.

It is clear, however, that such classes can provide a successful experience for girls as well as boys (Sukhnandam et al, 2000; Warrington \& Younger, 2003). Teachers in each school were clear that single-sex teaching offered clear opportunities and advantages for girls in their learning, promoting confidence about the subject matter and stimulating dialogue and collaboration. In each school within the triad, lesson observations suggested that the work ethic and level of motivation in girls-only classes was often more self-sustained by the girls themselves, and that classes were usually characterised by less explicit challenge to the teacher. Girls-only classes, though, sometimes present a more subtle, less tangible challenge to teachers, and a degree of spiteful behaviour (often described by girls themselves as 'bitchiness') which made them challenging contexts for teaching. In other contexts, there was a real danger that the surface calmness and order in all-girls classes hid under-achievement and off-task behaviour. Equally, then, the potential for single-sex classes for girls is only likely to be maximised where an explicit pedagogy is developed which recognises the need to stimulate and challenge girls in their learning, and which acknowledges the quiet invisibility of some girls in some classrooms (Warrington \& Younger, 2000).

It seems to us, therefore, that successful teaching in single-sex classes should be less concerned with developing explicit gender-specific pedagogies, and more concerned with teacher belief, attitude and expectation. The evidence emerging from this triad and from other case studies within the RBA project suggest that discussion of so-called 'boyfriendly' teaching strategies is, in fact, simply discussion about the essence of high quality teaching. We have not seen evidence, despite extensive classroom observations and numerous individual and focus group interviews with boys and girls, that such strategies support the learning of boys more than girls, and we do not accept the claim that girls' classes require a less active, less structured, less interactive, less varied pedagogy than boys' classes. 


\section{Preconditions for Implementation?}

Our research in these triad schools confirms that many boys and girls feel more comfortable in single-sex classes because of the lack of distraction of the other sex, and feel more able to question, explore issues related to learning and take part in discussion without fear of ridicule or embarrassment. Boys and girls experiencing such classes have consistently told us that they feel fewer pressures to perform, to 'showboat', once the other sex are not present, and that single-sex classes, in certain circumstances, have a positive effect on their learning. Equally, though, it has become clear that single-sex classes are no universal panacea. Teaching classes in such contexts can be demanding in classroom management terms, and behavioural issues can override all others. It seems to us, then, that if such classes are to contribute to achievement, a number of pre-conditions must be established and sustained:

- The development of teaching strategies to support such classes: whilst we do not accept the view that there is a boy-specific pedagogy which is different from a girlfriendly pedagogy, it is clear that single-sex classes do sometimes present more pedagogic and management challenges. It is essential, then, to develop teaching strategies, as we have outlined above, which engage and motivate these students, to ensure that the pedagogy employed makes it worthwhile and valuable for students to turn up to lessons.

- Teachers therefore need to be committed to this form of organisation in their subject, to have access to strategies which have proved effective in other contexts, and to have a means whereby they can exchange experiences without feeling threatened or undermined.

- This means that senior management within a school must embrace the single-sex approach, giving clear and unequivocal support, keeping parents / carers and students themselves fully informed of the rationale behind the school's approach, promoting the issue in a high profile way within the school community. Our experiences suggest that this proactive and supportive role of senior management, monitoring but also offering public support, is absolutely crucial if the potential of the initiative is to be maximised. Where senior management has been simply accommodating or even detached, the effects have been much more limited.

- In all three schools in this triad, the most effective teachers went beyond 'good practice' pedagogic strategies, to establish a sense of togetherness and common purpose which helped to sustain the credibility of the teacher and to maintain a collaborative sense of working. Humour and informality were used to motivate and engage students in learning, to generate collaboration and a sense of team spirit, and to consolidate a relationship of shared respect and commitment. Yet there was a further aspect of this mutuality of understanding, associated with definition of context. The most effective teaching in single-sex classes took place when common expectations had been clearly established and were accepted by all, when it was understood that disruptive behaviour or failure to complete work, especially homework and coursework, would not be tolerated. This mutuality of understanding created a context of high expectation, and particularly a climate in which boys particularly could perform without fear of undermining their own image or losing face with their friends of either sex. The mix of collaboration and 
engagement with persuasion and requirement enabled them to associate with and publicly acknowledge the aspirations of the school.

We are clear that such single-sex classes on their own are no panacea for the problems of poor behaviour, disaffection and lack of achievement, but equally we are clear that they can provide a positive and successful experience for girls and boys. Central to this, though, is the willingness to sustain, monitor and evaluate single-sex classes as a mode of organisation through time, and to develop a classroom pedagogy which is accessible and opens up learning for all students, regardless of gender. Whilst we accept Martino \& Meyenn's contention (2002) that 'productive pedagogy ... is not so much attributable to the single-sex strategy per se, but to the complex dimensions of ... teachers' pedagogical approaches' (Martino \& Meyenn, 2002, p 321), we do conclude that single-sex classrooms can offer opportunities to develop a more conducive classroom atmosphere for learning, in which effective teaching strategies can be better developed. In recognising this, however, we set our face firmly against a specifically 'boy-friendly' pedagogy, convinced that such an approach has been sustained neither by research nor by classroom experience, and demeans the worth of girls. 


\section{CHAPTER 8: Socio-Cultural Approaches: the Secondary School Context}

A fundamental starting point in any discussion of the factors which affect boys' academic performances at school must be to try to understand the rationale and motives which underpin the behaviour and (lack of) engagement of some boys. Our ongoing work with these secondary schools suggests that some boys underachieve academically because they identify with concepts of masculinity which are in conflict with the ethos of the school. As we discuss in chapter 1 , this relates specifically to a laddishness of behaviour, expressed in noise, in behaviour, and dress, as they seek to define their masculinity, and as they try to act in ways which are in line with peer group norms, in ways which protect their macho image (Francis, 2000; Skelton, 2001; Martino \& Pallotta-Chiarolli, 2003). The aspirations which the school has for them are not seen as acceptable because they can only be achieved by adopting strategies - particularly linked to academic work - which are divergent from those accepted within the peer group.

Avoidance of the feminine is also crucial in this protection of a macho image, so that some boys - keen to assert their heterosexuality - might engage in aggressive homophobic behaviour, while others might avoid academic work (or at least give the appearance of avoiding academic work) since to work hard at school seems to be perceived by many young people as a feminine activity.

In parallel with this, some boys protect their macho image and their social self-worth by making considerable efforts to avoid the culture of failure (Jackson, 2002, 2003). Such boys seek explanations - through their off-task behaviour, their self-protection through lack of effort in terms of classwork, homework and coursework, their lack of acceptance of the aims and objectives of the school - for their poor performance in school, to protect themselves against failure and competition. The possibility of failure can lead to anger, hostility and disaffection, and in turn to a self-sabotaging, anti-learning stance which can be expressed in physical anger, fighting and dominance.

\section{A Case Study}

Within any school, there are often a small number of students who exert influence within classrooms, who have a negative impact on learning, and who hold a key role in the local youth culture. In some schools, there is evidence of a growing number of girls within these disengaged sub-groups, but usually they are dominated by boys. Often they have a powerful influence which can 'knock-on' to other boys (and some girls) and have a significant impact upon the engagement of others within school. On occasions, they are on the margins of the school's academic and social life, with apparent anti-school traits and anti-social behaviour. Sometimes, they acknowledge this, and claim that they want a 'fresh start', but often they are unable to accept a new beginning, even when one is offered. Crucially, these students are strongly influential in establishing the peer group norm within a year group, whether it be linked to dress, behaviour, attitude to work or aspirations. How the school responds to them is crucial.

The RBA project has supported the development of socio-cultural approaches in one triad in attempts to combat the impact of these images of laddish masculinity on the learning of some boys, and to develop an ethos which helps to eradicate the 'it's not cool to learn' 
attitude amongst certain boys. Central to these socio-cultural approaches have been attempts to identify these key movers within the student body, and to incorporate them into the mainstream of the school or to reduce their influence over others by marginalising their impact. These socio-cultural strategies are not always overt or explicitly articulated, but tend to be implicit and integrated within the whole school culture, to develop an ethos which helps to identify and diminish the importance of anti-work groups; in the words of one headteacher, they are approaches which 'attempt to reframe the students' view of school so that academic success is valued, aspired to and seen to be attainable'.

These strategies have been developed in a group of mixed 11-16 comprehensive schools serving predominantly white working-class catchments in the inner city of a metropolitan area in North West England. Subsequent expansion of the triad, in a second phase of the Project's work, incorporated three further schools and increased the ethnic and socioeconomic diversity of the triad; one phase 2 school, for example, had $44 \%$ of its students eligible for free school meals, and another had 35\% of its intake with English as an additional language.

The Originator school had been identified during the pilot stage of the RBA project as one of the very few schools nationally where the gender gap had been narrowing significantly through time, and where there was an upward trajectory in the trend of achievement of both girls and boys. The school, established in its current form in 1990, had faced severe challenges in its early years of existence, with vandalism, violence, drug dealing and low levels of parental support and aspirations. In the words of the headteacher:

10 years ago we got $9 \% \mathrm{~A}-\mathrm{C}$ and were bottom of the league table. We were publicly vilified.

The socio-cultural approach implemented within the school has been underpinned by the single-minded determination of the headteacher and her SMG (Senior Management Group) to create an alternative learning culture for students once they are in school, which contrasts with the norms of the street culture within which many students normally operate. From the outset this acknowledged the extent to which a laddish masculinity and an anti-school ethos had gained ascendancy. There was also a strong belief that tackling the peer group culture was the most significant challenge facing the school if achievement was to be raised.

\section{The key leaders / key befrienders scheme}

Central to the socio-cultural initiatives adopted within the Originator school was the key leader / key befriender scheme, attempting to engage key image makers in year 11 and to incorporate them positively into the life of the school. A classification of key leaders in the Originator school suggests at least three different groups of students:

- The Rebels: intelligent students on the five $\mathrm{A}^{*}-\mathrm{C}$ borderline, usually though not exclusively boys with an occasional tendency to bullying and/or rebellious behaviour, and likely to mock others who work. Strong personalities who disrupt lessons easily and who intimidate some staff.

- The Clowns: usually immature boys who start inappropriate behaviour in classrooms; often incite other students, encourage and initiate truancy, and set dares and challenges for other students. Not necessarily popular, but sufficiently powerful so that other students want to keep on the right side of them. 
- The Stars: successful and popular, but not thought of as outsiders or swots; boys amongst them tend to be good at sport or have good interpersonal skills, and they are not afraid to help other students with their work.

Those key leaders - initially all boys, but latterly including some girls - are not always hostile and challenging, and continually set on conflict, but are seen by the school as being in need of encouragement and support to remain on track, having the potential to succeed and to become key role models for other students. Where the scheme is successful, key leaders are positively incorporated into the school's ethos, accepting that 'it's OK to work' and their expectations are raised. Crucially, too, the followers of the key leaders are brought onside as they observe the growing work ethic of key leaders, and there is an integration of the key leaders and their followers into the school ethos.

The process of identification of these key leaders (usually no more than twelve students) takes place towards the end of year 10 through staff and departmental meetings. All members of staff are made aware of who the key leaders are and are asked to pay them special attention to help them feel part of the school. Each is allocated a key befriender, usually a member of staff who relates well with the key leader, and acts as an informal mentor. The choice of key befrienders is made very carefully: not only does the Senior Management Group ensure that appropriate role models are chosen so that stereotypes are not perpetuated (so key befrienders are not physical or dominant), but key befrienders also have to be credible if they are going to get key leaders to complete coursework, attend extra lessons and work cooperatively within the school structure.

Key leaders are obviously aware that they have been targeted for support, but they are not explicitly aware of the school's rationale for doing so. The scheme tends to be perceived by students, therefore, as a form of tightly focused mentoring, and indeed it shares some of the characteristics of quality mentoring defined elsewhere in this report. The difference lies in the underpinning rationale of the approach, and in the cumulative impact which the school hopes the scheme has, not only on key individuals but also on their followers. The aim is to develop informal contacts with these students, through regular but unscheduled meetings which help motivate and sustain the students' involvement with school.

\section{The wider socio-cultural context}

Early attempts within the triad to transfer the essence of the key leaders strategy to the two Partner schools were not entirely successful. This focused the attention of the research team back to the Originator school, in an attempt to explore why the strategy worked in one context but was not immediately transferable to others. In so doing, it became clear that a number of pre-conditions were necessary.

In the Originator school, it was evident that very clear expectations were established by the headteacher and senior staff, relating to behaviour, attendance, punctuality, dress and appearance. We have defined these as proactive measures which make very clear what is expected of students as learners:

- A prompt start to the school day: no social time, no initial form tutorial period: academic learning takes precedence. 
- Behaviour monitoring via an electronic system which is readily accessible to all teachers: event logs enable SMG to identify issues with students as they develop during school day / week.

- Students who do not attend actively chased via telephone calls to parents; persistent non-attenders brought to school; electronic registration allows monitoring of students regularly throughout day, by teachers via laptops, to ensure that students remain at school once there.

- Uniform, dress code and school bags strictly implemented: students expected to come to school 'looking as though they intend to study'.

At the same time, it was recognised that students face barriers to learning which need to be removed to enable the students to succeed. So a series of support measures include:

- Additional twilight lessons and weekly breakfast clubs, focusing on coursework; structured revision schools in Y11 half term and at Easter; no Year 11 Study Leave prior to examinations.

- Guardian Angel Scheme: Y10 volunteers (through job descriptions, applications and subsequent training) working with Y7 tutor groups to help new students settle within school, to combat minor incidents of bullying and to offer specialist advice in particular areas such as drugs awareness.

- High profile teacher inputs in each lesson: direct pedagogic style to engage all students, making it worthwhile for them to attend each lesson.

- Learning community for staff: regular and systematic lesson observations, to identify models / exemplars of good practice; individual training plans for all staff.

We are aware that these various approaches are not new. What is different, perhaps, is that there is a holistic and coherent approach here to tackle laddishness, to establish an ethos for learning and to raise students' expectations, through an integrated approach. Students are convinced of what it is possible for them to aspire to, and given the means to achieve, through both coercion and support. Central to this socio-cultural approach is the attempt to create an achievement culture which offers students an alternative to the culture that often pervades the community and the locality, to challenge and attempt to modify the prevailing street culture. Students repeatedly and consistently buy into this alternative vision, as our interviews have shown. The following shows a conversation between an interviewer (I) and two year 10 boys (B1 and B2):

I: I'm getting the impression here, walking around, that achievement's really high profile in this school?

B1: Yer, they have the Achievement Thing most of the time.

B2: They don't say you have to do well.

B1: They give us a choice, but they put it one way. They just try to explain it and make it sound like a good idea.

B2: They like to tell us achievement, if we don't have achievement we won't like get a good job or anything.

B1: Yer, that's another thing. In assembly they go 'If you don't get your GCSEs you're going to end up at Pandora's Pickles' or something. 


\section{Impact of socio-cultural strategies}

The key leaders / key befrienders scheme, together with the series of proactive and supporting measures described above, is one of the main strategies which has helped to change attitudes to schooling and attainment patterns in the Originator school. Attendance rates have increased, exclusion rates have dropped, and levels of attainment at GCSE have risen consistently. In interview, students have spoken of being motivated and feeling valued as a result of the key leaders scheme:

She talks to me and stuff and she helps me along. It's not like they're doing it to get at me. They're doing it for my benefit so it's ok: they're doing little things that will help me keep on the straight and narrow. If you don't do well in school, you're not going to get a decent job and a nice house.

I've changed this year. I'm trying to do work. I didn't try last year. I've changed because I've realised I've only got a few months left until I leave. I want to do well, get good grades, for what I want to do anyway. I want to do graphic design at college and I need a $C$ in everything.

Students had become convinced of the need for qualifications to 'break out of cycle of poverty in this area' and felt treated in an adult and positive way by the school; the school was 'alright', and all the key leaders interviewed echoed the view that the school wanted them to do well, and that the headteacher was 'a fantastic woman who was 'right on'!!

Key befrienders echoed these views, quoting the levels of qualifications gained by many key leaders and their followers, their subsequent career aspirations and their determination to progress through to further and higher education. In the words of one experienced key befriender:

Over the years there has been a considerable success rate. A kid from three years ago was here being interviewed for the lab tech job the other day. Another one from the same year is at Salford University. They know that if they leave school with nothing, then when they emerge from the bad times they have nothing. By working with them they don't leave here with nothing.

It was suggested, too, that key leaders who rejected the support the school offered were in turn being ostracised by those who had embraced the ethos of the scheme.

Attainment data over the last decade supports the perceptions of key leaders and their befrienders. Figure 8.1 shows the full extent of the transformation, achieved within a stable and challenging catchment, and significantly, without marked changes to the curriculum structure offered within the school. The key leaders scheme was developed initially in 1998, and although we must stress again that its success is dependent upon the overall context in which it operates, the changes in the pattern of attainment since then are remarkable. Although the gender gap has not been eliminated, there are years when boys have outperformed girls, as in 1998, 1999 and 2001. More significant, however, and of more value, has been the surge in attainment. Although the gender gap has reopened in some years, this must be seen alongside the dramatic acceleration in the rate of girls' achievements in recent years and the trend in the boys' results. Indeed, in 2004, the highest ever proportion of boys and girls achieved GCSE grades at the higher levels, against a gender gap of only $3 \%$. Given the catchment area of the school, this is an astonishing transformation. 
Fig.8.1: GCSE Results by Gender at Originator School

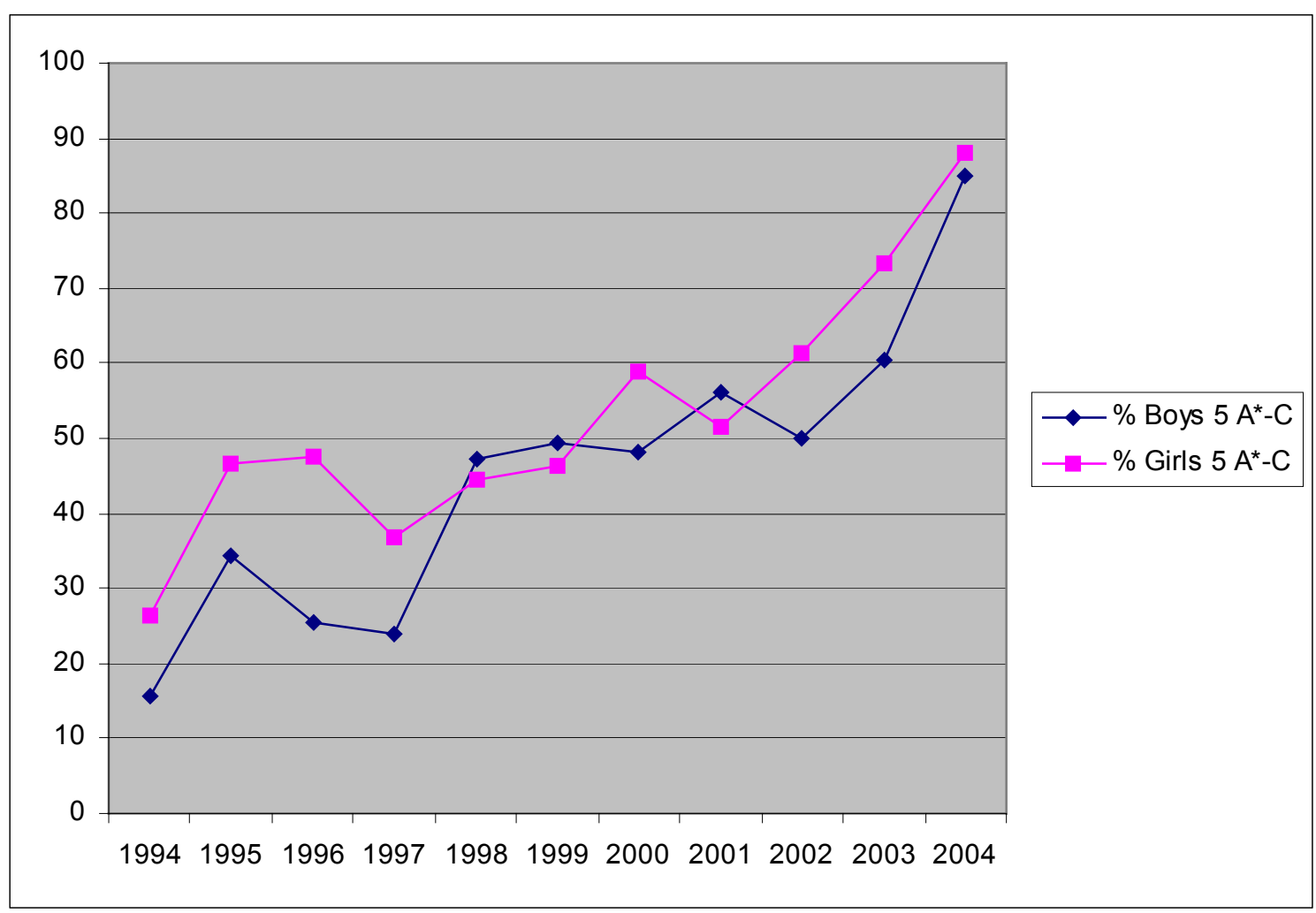

\section{The wider triad}

As indicated above, the process involved in transferring the essence of this key leader / key befriender scheme to the Partner schools proved to be complex and intricate. Both Partner schools had similar catchments and faced similar challenges to those in the Originator, but both schools differed in priorities and organisation. It soon became apparent that the intervention strategy rested heavily on one member of the SMG in each school, that there was benign interest rather than active support from other SMG members and crucially from the headteacher, and that there were problems securing key befrienders who had appropriate skills and rapport. In both schools, however, there was some reluctance to identify with the aims of the scheme, and antipathy to a scheme which sought to reward and give special attention to students who were often hostile and challenging in classrooms.

The scheme was launched, then, around key befrienders who were either members of the SMG, often closely associated with a disciplinary function, or Learning Mentors, who sometimes lacked both status and credibility in the eyes of staff and students. Sometimes, too, the key leaders chosen were openly and consistently hostile to the school, and confrontational in all aspects of their behaviour and attitude, and it became a very difficult task to bring these students on board in the way the scheme had envisaged. In retrospect, we had failed at this stage to define specifically enough the essential characteristics of key leaders, with too much emphasis placed perhaps on behavioural characteristics. Had we done so, the scheme might have had more success with the particular individuals identified, and also been more acceptable to staff in these two schools. Despite the 
considerable efforts of both school coordinators, the first year was not one of success in these Partner schools, so much so that one Partner school withdrew at the end of the year.

The key leader strategy was more successful in the other Partner school as it became established during the second year, although it had less widespread impact than in the Originator school. This is hardly surprising, given the time that it takes to establish firmly any new intervention. Nevertheless, a number of positive outcomes were discernible, with interviews with key leaders revealing a number of promising trends:

- A recognition that they were not achieving as well as they could;

- A willingness to seek out help with their learning in some subjects where teachers seemed to be more approachable;

- Higher aspirations, and a more realistic awareness of what they needed to do at school to make these aspirations achievable;

- With some students, a real attempt to control their own behaviour, and to avoid conflict.

It was evident, that some of these students were attending school more regularly, and disciplinary referrals for them reduced. Their predicted grades began to improve, albeit from a low starting point, and although the overall percentage of students achieving the benchmark grades did not change significantly, there was a marked rise in the percentage of students gaining $1 \mathrm{~A}^{*}-\mathrm{G}$ grades at GCSE.

In the third year of the Project's life, the key leaders intervention strategy was extended to three new schools in the same geographical area. Although this extension was not long enough to enable us to evaluate the impact of the strategy upon performance data, we were able to consider the impact of the strategy upon students' attitudes. An attitudinal survey was completed by students in each school, (the Originator, the Partner and the three extension schools) at the beginning and end of their year 11 schooling (Table 8.1). Three significant aspects of students' attitudes (their engagement with school, their sense of self as student and their academic self-concept) were identified and assessed.

In each case, there is a statistically significant upward trend in the scores recorded by the key leaders and by their followers, greater than that recorded in the year group as a whole. We must be somewhat cautious in interpreting this data, because of the small sample size of key leaders and their followers, and because of inter-school variations, but nonetheless, there are some interesting outcomes. This analysis suggests, even so, that the key leaders did not disengage during year 11, as their prior behaviour suggested they might, and that their followers became more engaged with their schooling, saw themselves more as students and developed more confidence as learners during the course of the year. This does indicate that the schools, taken together, were becoming more successful in transforming students' culture and generating more appropriate attitudes and behaviours in their Year 11 students. 
Table 8.1: Engagement of male Key Leaders and their Followers

\begin{tabular}{|c|c|c|c|c|c|c|}
\hline & \multicolumn{2}{|c|}{$\begin{array}{l}\text { Students' Engagement / } \\
\text { perceived support from } \\
\text { teachers }\end{array}$} & \multicolumn{2}{|c|}{$\begin{array}{c}\text { Students' sense of self: } \\
\text { behaving appropriately as a } \\
\text { student }\end{array}$} & \multicolumn{2}{|c|}{$\begin{array}{l}\text { Students' academic self- } \\
\text { concept }\end{array}$} \\
\hline & \multicolumn{2}{|c|}{$\begin{array}{l}\text { Possible range of scores } 15 \\
\qquad-60\end{array}$} & \multicolumn{2}{|c|}{$\begin{array}{c}\text { Possible range of scores } 7 \\
-28\end{array}$} & \multicolumn{2}{|c|}{$\begin{array}{c}\text { Possible range of scores } 6- \\
24\end{array}$} \\
\hline & $\begin{array}{l}\text { Mean scores } \\
\text { at start of } \\
\text { year } 11\end{array}$ & $\begin{array}{l}\text { Mean scores } \\
\text { at end of year } \\
11\end{array}$ & $\begin{array}{l}\text { Mean scores } \\
\text { at start of } \\
\text { year } 11\end{array}$ & $\begin{array}{l}\text { Mean scores } \\
\text { at end of } \\
\text { year } 11\end{array}$ & $\begin{array}{l}\text { Mean } \\
\text { scores at } \\
\text { start of } \\
\text { year } 11\end{array}$ & $\begin{array}{c}\text { Mean scores } \\
\text { at end of year } \\
11\end{array}$ \\
\hline $\begin{array}{c}\text { Key } \\
\text { Leaders }\end{array}$ & 34.2 & 39.3 & 16.7 & 18.5 & 13.9 & 15.5 \\
\hline Followers & 32.8 & 38.3 & 17.0 & 19.0 & 14.0 & 15.3 \\
\hline $\begin{array}{c}\text { Other } \\
\text { students }\end{array}$ & 38.5 & 38.7 & 20.7 & 20.7 & 15.7 & 15.9 \\
\hline
\end{tabular}

The extension of the project also enabled us to consider further the conditions which need to be established in schools if the potential of such a directed strategy is to be exploited to the full. It is to a discussion of these pre-conditions that we turn now.

\section{Pre-conditions for Successful Implementation of Socio-Cultural Strategies}

Attempts within these schools to address issues of laddishness have met a number of barriers. This is hardly surprising, given the complexity of sociological and psychological issues surrounding laddishness, and the socio-economic context in which the schools in this area are located. Some of them, often trying to work in response to a myriad of different initiatives, have been unable to address fundamental issues:

- Identifying possibilities for achievement. Within two of the six schools we worked with there was consistent under-achievement, particularly of boys, and an acceptance of historic levels of achievement which were significantly below that suggested by the students' prior attainment data. Value-added data in one school, for example, suggested that $45 \%$ of the year 11 students' cohort had the potential to achieve the benchmark grades, compared to the $33 \%$ which the school regularly achieved. A minority of staff in this school appeared to have a strong influence on school culture and expectations, and were a powerful influence on the (lack of) achievement culture established. Suggestions that higher levels of attainment should be aspired to and could be achieved were dismissed as unrealistic.

- Failure to transform aspirations: some of these schools were failing to challenge the low level of educational aspirations within their communities. Given high unemployment levels, the prevalence of traditional employment opportunities within the informal sector and within family networks, and the past failures of the education system, this is hardly surprising. In some sectors of these communities, there seems to be little sense in ensuring that children attend school when there are 
opportunities to earn and to prosper in various ways in the informal sector, and when what education offers is perceived to be dull and oppressive.

- Staff retention: the challenges faced by many schools in these localities are welldocumented, but retaining qualified and committed teaching staff is one of the most pressing. Socio-cultural intervention strategies are complex and need time to embed; crucially, there needs to be a commitment to them from the staff, particularly from key befrienders, and a clear understanding of rationale and process. In some schools, staff recruitment and retention have been pressing issues which have destabilised the intervention strategies, and it has been difficult to sustain them through time in the face of staff long-term absences, extensive dependence on supply staff and unfilled vacancies.

There is a clear counter-balancing, however, in schools within this triad where these challenges have been successfully addressed. As we outline above, in the wider sociocultural context, there are proactive measures which have been put in place which help to create and establish an achievement culture within the school. Creating a context which makes it obvious to students and their carer(s) that the school is essentially a place for working and for learning is a crucial starting point. There is nothing startling here; yet the ability to establish context and to define what is expected, and to have the charisma and determination to offer leadership, is a crucial pre-condition which must be met if expectations and aspirations, of staff as well as students, are to be raised. Then, in the words of one headteacher, students can be assured that at school 'you can work and not be name-called for it, in a place where it can be cool to be clever'.

Equally, research in this triad suggests that students also need clear support systems which offer guidance and help on the one hand, and initially at least, prescription or strong persuasion on the other. There is a sensitive balance here between offering additional support such as twilight clubs for homework and coursework, and ensuring that those students who need to attend do actually attend. This may mean setting explicit targets for attendance in the first instance, but students will only attend if they can go without loss of face.

\section{Closing Perspectives}

During the final year of the RBA project, we have discussed these socio-cultural issues extensively with headteachers and key school staff who have worked closely with us over the life span of this Project. In particular, we have tried to decontextualise the key issues within the debate, and to identify the essence of socio-cultural issues which might impinge on schools wherever their geographical location and whatever the socio-economic characteristics of their catchments. On the basis of these discussions, we would suggest that, to be effective in addressing issues of laddishness and an emerging 'ladettishness', such socio-cultural interventions need:

- To have an emphasis on persuasion, support and confidence-building; to help some boys and girls establish more self-esteem and confidence as learners, because whilst some of these boys and girls certainly do not lack social self-esteem within their own peer groups, they do lack self-esteem as learners;

- To open up possibilities of achievement which might not be part of the community or peer group expectation, to use target-setting and mentoring to offer visions of 
what is possible, to raise aspirations and expectations, thereby offering choice and conferring responsibility;

- To focus upon individuals, so those key boys and key girls who help to establish the ethos and tone of the year group, and who help to set peer group expectations, are identified. Where these students are disaffected, some schools will find the key leader / key befriender scheme an important support in bringing these students more centrally onboard, and in helping them resist peer group norms of anti-work. In other schools, more implicit mentoring may achieve the same result. Whatever the approach, these students are key, and schools - in our experience - need to give more thought to explicitly addressing the image and attitudes which they display, and offering support and direction to them in a collaborative way. 


\section{CHAPTER 9: Socio-cultural Approaches: the Primary School Context}

Whilst socio-cultural issues come to the fore in secondary schools, it would be a mistake to believe they were not also relevant in primary schools. As Reay (2001, p.157) notes,

'the 'failing boys' compensatory culture of aggressive 'laddism' has already started to be played out at the micro-level of the primary classroom'.

Pressures for gender conformity, as Jordan (1995) found in her research in Australian primary classrooms, are much stronger on boys than on girls. Thus even at primary level boys are seeking to construct a form of masculinity through which they can acquire popularity and status within their peer groups (Swain, 2003, 2004). Boys also, Hey et al (2000) suggest, experience contradictions between the cultural messages associated with hegemonic masculinities and those teaching practices conducive to optimal learning in primary schools. In this instance, their competitive behaviour militates against their achievements, so that when 'wannabe' hegemonic boys do not 'win', they tend to adopt a 'can't win, won't win and don't want to play' stance. Even very young boys, Lucey et al (2003) suggest, will avoid working hard in order not to be seen as a 'geek'.

As Swain (2003) suggests, there are different gender regimes operating in different places, with each setting having its own dominant or hegemonic form of masculinity. It is thus important, as Skelton (1996) points out, to consider the nature of social relations within a given local community, and to take into account the roles of class and ethnicity in shaping particular masculinities in specific places. It is as true at primary as at secondary level, that in some places there are considerable tensions between the culture of the school and that of the local community from which the school draws its pupils. While in some schools, only a small number of boys conform to an image which negatively affects their engagement in school and learning, in other places a larger group of older boys can have considerable influence within their peer group and school, and have a detrimental effect on ethos and achievement.

Socio-cultural strategies had a high profile during the pilot phase of the RBA primary project, and also provided the focus for three triads in the main intervention stage, where two used a whole school approach, while the other involved particular year groups, and was more classroom-based. Once each of the triads had agreed the precise focus of the strategy to be followed in their schools, a group of between eight and twelve boys was selected for tracking by a researcher through their final two years of primary school. They were interviewed on average on five occasions, either singly or in groups of two or three, and details of their progress were obtained from their class teachers. Apart from in one Originator school (see below) these boys were felt by their teachers to be 'underachievers', in the sense that all were felt to be academically capable of doing better, but their attitude, lack of engagement or poor behaviour impeded learning. They were described, for example, in the following terms:

He's easily led - slouches around with a cool boy attitude - he's concerned about how others perceive him. He has a good brain, but he's not using it - can, but won't.

He has a negative attitude to school. He distracts others and does not engage in a cooperative way. 
The boys described as under-achieving were usually either key leaders among their peer group, distracting other children, or they were boys who were themselves easily distracted. Often they were described as able children who put little effort into their school work. Several of them had problems outside school which were felt to affect levels of engagement and achievement. The focus of the strategy, the process of implementation and an analysis of the outcomes in each triad are discussed below.

\section{Socio-cultural Strategies through a Whole-School Approach}

The rationale for the two triads engaged in a whole-school approach was the belief that engendering involvement in school, and explicitly encouraging all children to be as fully engaged in all aspects of school life as possible, means that they are less likely to turn away, and feel alienated from learning. Many primary age children do, of course, feel involved in school and learning, but as the previous discussion indicates, there are groups of children, often boys who, even at that stage in their education, feel that 'it's not cool to learn', and experience conflict between the expectations of their teachers and those of their peer group. The approaches which were undertaken in the project schools were many and varied, and included a range of initiatives put in place to create a more inclusive school environment and to raise children's self-esteem as learners. Some, such as school councils, school plays and circle times are familiar to many primary schools, while others, such as You Can Do It (see below), Black History month, classroom buddies and teambuilding clubs are perhaps less common. All of them can have an impact on children's engagement with school. For example, as one year 5 girl, reflecting on the introduction of a school council two terms previously, explained:

The school council's definitely a good thing. It gets us involved - gets children talking about things, and the councillors have done really really well.

A mixed ability group of year 5 and 6 girls and boys in one school were unanimous about the positive impact of Poetry Week, which was seen by all as fun, engendering a sense of community as well as confidence, as children worked in teams to produce poems for presentation in special assemblies. In another school, children talked not only of what they learnt about multiculturalism, but of the enjoyment they experienced during Black History month, engaging in various activities from making puppets and hand-painting, to watching visiting groups tell stories.

What is important, however, is that such activities are framed within a context where the creation of a positive whole school ethos ensures that each child is made to feel valued and special within a culture of equality, teamwork and high expectations. This was explicit in all the schools where these kinds of socio-cultural initiatives were working well. Thus as a boy from one of the Originator schools explained:

When I'm not here the teacher says she misses my ideas, so I always feel I'm part of the school.

But what of the particular impacts on the under-achieving boys who have been the main concern of the Raising Boys' Achievement Project? Have such initiatives helped them to feel more involved in school life, and has this involvement led to changing attitudes towards school and to an improvement in academic attainment? It is not possible in a report of this length to discuss the full range of initiatives used in the project schools: 
rather, we focus on three case studies, chosen to illustrate ways in which particular approaches are impacting on under-achieving boys.

\section{Case Study 1}

\section{The background}

The schools in this triad were located in a predominantly working class urban catchment area in the West Midlands. Significant proportions of children in each school had a first language other than English, and the proportion of children eligible for free school meals was above the national average. Both Partner schools also had above average proportions of pupils identified as having special educational needs and parental expectations were low. Performance at Key Stage 2 in the Originator school had consistently exceeded the national average in all three core subjects, and there had been a steady increase in boys' performance. Although there had been overall improvement in results in both Partner schools, performance in national curriculum tests in all core subjects at key stage two was well below the average for similar schools, though in Partner school 1 boys had done well since 1999, with their performance significantly outweighing that of girls. The other Partner school, situated in a particularly deprived catchment area, had recently been in Special Measures, and had considerable problems with staff recruitment and retention.

\section{The strategy: a focus on citizenship}

Intervention strategies in this triad were linked to work in Personal, Social and Health Education. A range of approaches, broadly falling under the aegis of citizenship education was put in place, the main initiatives being school councils, circle time, 'You Can Do It', classroom buddies and special pupil responsibilities. These were evaluated through pupil and teacher interviews, observation and measuring attitude change, and whilst all the initiatives were found to be important in contributing to the inclusive ethos mentioned above, the two initiatives having the most impact on the under-achieving boys in the focus groups were Circle Time and 'You Can Do It' (YCDI), which we use as exemplars here of two successful approaches.

Circle Time is a common activity in primary schools, and was part of existing practice in all the West Midlands schools when the triad came together, but a decision was taken in 2001 to re-orientate Circle Time as an essential classroom-based forum for the discussion of issues relating to the School Councils which themselves provided the focus for the citizenship initiatives. What became distinctive about Circle Time in these schools was not simply that training and resources were prioritised, but that they were seen as part of a wider package of activities and given high status. In addition, staff were actively encouraged to monitor and ensure the participation of under-achieving boys during Circle Time; as one commented:

Teachers use planned activities and focused questioning to ensure all pupils are engaged and feel included and valued.

In both schools, Friday afternoons were designated as PSHE afternoons, or 'Golden Time' with a specific time allocated for Circle Time, and YCDI also forming an important part. Asked how such activities were fitted into the timetable, the member of staff with responsibility for implementing the citizenship initiatives responded: 
We make time because we think it's valuable. If you can justify it, you make time, don't you?

One of the West Midlands Partner schools had been part of a pilot scheme for YCDI, the scheme adopted by the Local Education Authority as part of its Personal Social and Health Education programme. Developed by Michael Bernard (Bernard and Hajzler, 1987), YCDI identifies the four 'mind sets' of confidence, persistence, organisation and getting along, which are seen as providing the foundations for achievement and social-emotionalbehavioural well-being in school. The scheme also identifies four 'blockers' - low selfesteem-anxiety, general work avoidance, general disorganisation and rebelliouness-anger which are seen as contributing to poor psychological health, under-achievement and disaffection. Commenting on the scheme, a teacher said:

It's all about recognising who you are and beginning to value yourself, which for the 'classic underachiever' has a positive impact.

Given its success in the Partner school, the other two triad schools decided to incorporate YCDI into their citizenship initiatives: INSET training was arranged, and the scheme implemented in the autumn of 2002.

One of the main aims of citizenship initiatives as a whole was to try to build an inclusive ethos through giving children a voice. This involves a certain amount of risk-taking, since pupils make their own choices and decisions, but also, crucially, through a supportive framework of YCDI, which provides children with the necessary skills for managing choice, and Circle Time, which gives them opportunities to discuss their choices and the consequences for themselves and for other people.

\section{Assessing progress}

Whilst it is not possible to isolate a set of strategies and to hold them solely responsible for any change in pupils' engagement in school, increased self esteem for learning, or improved achievement, both the schools and the project team were confident that the citizenship initiatives were having positive effects. When the project's intervention stage finished, most of the strategies had been in place for a relatively short time - some for only a year, but it was clear at a meeting with the Originator and Partner 1 a year later, that the strategies were becoming embedded. In the words of the Acting Headteacher of Partner school 1, 'they're now just a normal part of our school life'. As this occurs, it is likely that the benefits for under-achievers will increase, but even at the end of the intervention stage, these were already apparent.

Firstly, there was qualitative evidence of increased levels of engagement and more positive attitudes towards school. As one class teacher commented, in relation to one of the focus group boy's participation in Circle Time:

He has been inattentive and disengaged, but he is now becoming a much more interested participant in discussions, though he still sometimes distracts others and does not engage in a co-operative way.

Thus, whilst managing Circle Time needed particular skills, teachers felt that it allowed the under-achieving boys to become engaged in issues relevant to them, and it was felt that the focus group boys across all three schools responded well. The boys themselves also spoke positively about Circle Time, suggesting it was a time when they could tell the truth, speak 
out and resolve problems. They felt that Circle Time had led to a greater degree of openness between themselves and their teachers, so that relationships had improved. They also enjoyed the opportunity to express their ideas orally, rather than in writing, and by the final interviews they were noticeably more able to articulate their feelings.

YCDI was seen as effective, too, in providing children with regular opportunities to become involved in non-academic lessons that focused on promoting positive self-images, engaged their interest and drew on their experiences. It was seen to have a significant impact in these schools because of the wholehearted commitment of staff to reinforce aspects of the scheme across the whole curriculum, reinforcing the language of YCDI in the classroom and through assemblies. This led to increased levels of confidence among this group of boys; in the words of one focus group boy:

It helps you to be confident and get to know people better, and not to be nervous.

The boys talked enthusiastically about the positive and negative statements of YCDI, and even those who claimed not to have enjoyed it, had clearly been influenced by its positive language. They were able to identify which 'minds sets' they were best at, which required improvement, and how they could improve. They felt, too, that the programme helped with organisational skills, which they recognised as being important for academic improvement, and they had also learnt how to control their behaviour better.

Quantitative evidence of change was also evident from the attitude survey (see below) and in terms of academic achievement among the focus group boys. Tables produced by the DfES (2003) allow a 'value-added' score to be calculated to measure progress for each child based on performance in core subjects from Key Stage 1 to Key Stage 2. The average value-added score for the focus group boys can then be compared with that for the school as a whole, and whilst the resulting figures must be treated with caution because of the very small size of the sample group of boys, they do show (Table 9.1) that, despite the initial identification of these boys as under-achieving, they had in fact made good progress between the two key stages. In Partner 1, and particularly in the Originator school, the focus group boys progressed at a greater rate than children in the school(s) as a whole, while in Partner 2, the progress made by the focus group boys was at a similar rate to other children, which was itself in line with the national average (between 99.3 and 100.7), despite their apparent 'under-achievement' in other contexts.

\section{Table 9.1: Key Stage 1 to Key Stage 2 Attainment for West Midlands Focus Group Boys}

\begin{tabular}{|c|c|c|c|}
\hline Value Added Measure & Originator School & Partner 1 & Partner 2 \\
\hline Whole school & 100.5 & 101.2 & 99.3 \\
\hline Focus group boys & 105.0 & 101.5 & 99.2 \\
\hline
\end{tabular}

Together these findings do seem to bear out the confidence in socio-cultural approaches expressed by the deputy headteacher in the Originator school:

We've tried all that before for kids who weren't achieving - give them more literacy, give them more numeracy - we've tried that and it doesn't work - they just get 
turned off. We really are introducing these socio-cultural strategies for them, whatever way we can, giving them opportunities to make better choice.

In essence, such strategies are, we believe, providing the underlying context for academic success.

\section{Case Study 2}

\section{The background}

Schools in the second case study were located in a deprived South London borough, where all served large local authority housing estates. Over 60 per cent of pupils in the Originator school were eligible for free school meals and almost half did not have English as their first language. The two Partner schools also had above average proportions of children in these categories, although Partner school 2 had more of a mixed intake, with children from private housing nearby. Despite its deprived location, the percentage of boys in the Originator school gaining level 4 in Key Stage Two English rose from 48 per cent in 1996 to 89 per cent in 2000, and in the three years 1997-2001, the performance of girls and boys in all core subjects at Key Stage 2 exceeded the national average by 1.2 points, while the average gender gap in Key Stage 2 English over the previous 5 years was only 0.1 in favour of girls, compared to a national average of 1.6. Both of the Partner schools also performed well in national curriculum tests, with results in core subjects at key stage 2 generally well above the national average for schools in similar circumstances, although both had a more significant gender gap in favour of girls.

\section{The strategy: a focus on the arts}

Each school already had a commitment to a broad curriculum, with a strong emphasis on the arts, particularly music and drama, and therefore chose this as the main focus for the intervention strategy. Like the citizenship activities outlined above, a wide range of activities was engaged in, such as artists-in-residence schemes, individual music lessons, visits to galleries and exhibitions, poetry weeks and school plays, and such activities will be familiar to all teachers. But while in many schools they are additional to the formal curriculum, in these three schools they were prioritised, and a significant amount of time was also devoted to them in the curriculum. For example, in Partner school 2, a whole week each year was devoted principally to poetry, with special assemblies, performances and classroom-based activities. During one term of the project, Partner school 1 engaged a series of dancers to run innovative weekly afternoon dance sessions for all classes. Thus arts-based activities were a central, rather than a peripheral part of the curriculum.

The Originator school had for some time used the wider curriculum as part of a strategy to involve all children in school life, and to help particular children to become more engaged, by targeting them for specific activities, such as lessons on a musical instrument or a lead role in a play. Thus by year 5 under-achievement was unusual, and the focus group in this instance therefore comprised boys of varying levels of ability, who, despite difficulties in their home lives which might have affected their learning, were achieving 'against the odds'.

At the start of the project, Partner school 1 was also keenly involved in arts activities, but, despite various initiatives primarily concerned with literacy, there was still a concern with the under-achievement of a small group of boys within the school. The Headteacher had 
long been convinced of the value of the arts in stimulating engagement in under-achieving children, and the focus of the strategy in this instance, therefore, was to track a group of nine such boys through years 5 and 6 , and to monitor their participation in various activities. In Partner school 2, while the arts were seen as an important way of involving children in school, they were not used to target under-achieving boys, as in the other two schools.

\section{Assessing progress}

A study by Downing, Johnson and Kaur (2003) suggests that the arts can contribute to increased motivation, behaviour, attendance and self-esteem, and are essential to raising standards in school. These findings support those of the Project, where we contend that the arts-based strategies not only benefited pupils across each school, but were able to have a particular impact on under-achievers where they were targeted to take part in particular activities. At the start of the project, the focus group boys in the Partner schools were described by their teachers as lacking in self-confidence and involvement in learning; they were frequently volatile, and found concentration difficult. Several of the boys themselves, in the initial interviews, were aware that they did not work as hard as they might, and were easily distracted:

I could probably improve a little bit because I do get told off quite a bit for not concentrating hard enough.

Boys can't think if people are talking - they stop and forget to get on with their work Listening to the teacher and working harder would help me to do better.

Tracking the boys across years 5 and 6 enabled the project researcher to talk to them about their involvement in arts activities, their responses to these activities and their engagement in school generally.

In the Originator school, where the gender gap was negligible, and where the arts had been used for a number of years as a way of generating involvement among disengaged children, initial interviews showed that the focus group boys did feel involved in school. One of them, who had had considerable emotional and behavioural problems, had found that having a lead role in the class play had proved really motivating, so that, according to the Headteacher, he had become 'a totally different child, completely focused on his work.' The boy himself was hugely enthusiastic, expressing a desire to go to a drama school, and being really proud of his achievements.

The clearest impact on under-achievement during the main phase of the Project was in Partner School 1. In their second term of year 5, pupils were involved in a production of Twelfth Night at the Globe Theatre, London, and four of the focus group boys had major speaking parts in the play. They were chosen, not because of their acting ability, but because they were perceived by their teachers to need greater confidence or higher selfesteem. All were surprised at being selected, which in itself proved a real boost to their confidence:

I didn't think I'd be chosen because I'm not very good. I thought my teacher had made a mistake, but when I got in the play I just wanted to jump up and burst out laughing. 
Once chosen, they were given extra support to enable them to learn their lines, and they talked about the patience needed for this and for rehearsing the same scene again and again. They talked too about learning how to express themselves and use their voices in different ways. Finding they could be good at something made them feel more confident in relation to their peers and their families, and being able to perform well therefore gave them a real sense of achievement:

I learnt all the lines and it went well. I felt very nervous beforehand, but I felt proud afterwards. Acting gives you a sense of boldness.

Even a year later they could reflect on their experiences, and articulate what they had learnt. As one explained:

I remember the kind of emotion when I was at the Globe theatre. It taught me that it does really really good to really express my emotion. I felt cold with fear beforehand, but afterwards I felt like I'd revealed everything and had expressed myself in front of everyone.

Another activity involving all the pupils was a series of dance projects, which, despite their initial reservations, all the focus group boys enjoyed immensely:

I didn't think I really wanted to do it, because I haven't ever considered dancing as something which I do. But the street dancing was fun, and the man who did it was really calm, so I enjoyed it much more than I thought I would.

I didn't really want to do it, and I was nervous in case I couldn't do it right, but it was kind of good when you got into it, because there were people from outside, and I kind of want to do it again.

I thought, oh no, not dancing, but when I came in and saw people playing the Brazilian instruments and they were in work-out suits, I thought, this is going to be really great, and it was.

Not only did they talk of learning organisational skills and working cooperatively with other people, but some found a direct effect on class work because, as one explained:

When we do things like that, and I come back to the classroom, I think, that was done with the school, and I've started to think I really like school because if our teacher can organise really fun things like that, she knows what children are like, and I think, that's good.

The third activity these boys were involved in was music: four had been selected to be in the Djembe drumming club, and three were in the school's steel band. Again, they had not expected to be selected from among the large numbers wanting to take part in these activities; as with the acting, they talked about the nervousness before performance, the concentration needed, and the sense of achievement at having performed well, and the importance of working together in a team:

When I'm playing it's really exciting. This morning in assembly when I was about to do the piece I was really scared, and my heart was pounding fast, because we all thought we were going to do something wrong. But you learn to concentrate, and listen to what the pans are doing. You have to care about other people and not just yourself, so you learn to cooperate.

What was really striking among this group of boys was the extent to which they changed between the first interviews at the beginning of year 5, and the last ones at the end of year 
6. Not only were the changes that had occurred evident to the interviewer, but all could articulate changes they had seen in themselves. Several commented about how their behaviour had improved and how they had become calmer, or more mature:

I'm kind of more mature, and I'm doing better with my work now. I listen a lot more than I did'; 'I've grown more mature, I've been thinking about what I'll do in life, and how I'll make progress; 'I've grown maturely and intellectually, and I've got smarter.

They talked too about increased confidence, and of a more serious attitude towards school work, realising that it was more important, and getting on with it even when they found it difficult.

In contrast, at Partner school 2, involvement in arts activities was seen as a broader approach, and not as a strategy for engaging under-achieving pupils. They were not targeted to take part in such activities in the classroom, and participation was more on a voluntary basis, with pupils choosing, for example, whether to put themselves forward for auditions. While those among the focus group who had been involved in drama and music activities were enthusiastic about them, only two had had speaking parts in plays, and half said they did not like either drama or music and would not wish to take part in activities outside the classroom. Thus in this school, although the focus on the arts could be seen as contributing to the fact that attitudes among boys in year 5, as measured through the attitude survey (see below), were the most positive of all the project schools, the benefits appeared not to impact on the focus group of under-achieving boys. As a group, qualitative evidence showed there to be less change between years 5 and 6 than in the other two schools, and although all said they had worked harder during year 6 , only half felt their work had improved. Three of the boys remained quite uncommunicative, and two were very negative about school.

The focus group boys from the Originator and Partner school 1, on the other hand, where arts-based activities were used specifically to generate involvement among underachieving pupils, were more likely to express positive feelings about school at the end of year 6. Furthermore, in Partner school 1, where the targeting of under-achieving boys in arts projects became a major focus from mid way through year 5, the boys made significant academic progress, as Table 9.2 shows.

Table 9.2: Key Stage 1 to Key Stage 2 Attainment for South London Focus Group Boys

\begin{tabular}{|c|c|c|c|}
\hline Value Added Measure & Originator School & Partner 1 & Partner 2 \\
\hline Whole school & 100.8 & 99.2 & 99.9 \\
\hline Focus group boys & 100.9 & 101.3 & 99.7 \\
\hline
\end{tabular}

Clearly, as with the first case study, other things were going on in these schools, and we would not wish to over-simplify. Nevertheless, the ongoing success of the Originator school, both in terms of pupils' engagement with school, together with the very evident changes in the boys in Partner school 1, do point to the importance of the arts in stimulating engagement in these schools, within a context of inclusion and teamwork. In Partner school 2, this ethos was important too, and the arts had a high profile, but while overall achievement was higher than average for similar schools, the lack of focus on 
individual under-achieving boys limited the effectiveness on achievement and motivation among such boys.

\section{A socio-cultural strategy through a classroom-based approach}

While the focus of the two triads discussed above was on involvement in school leading to involvement in learning, through a whole-school approach, a third triad in the RBA project showed that it is also possible to implement socio-cultural strategies at the level of the classroom. In this instance the aim was to transform learning, through a strategy designed to enhance pupils' perceptions of themselves as learners and to promote academic selfesteem. As with the strategies described above, such an approach works best where there is a whole-school ethos which explicitly celebrates success, which values individual pupils and which gives priority to teamwork.

\section{Case Study 3}

\section{The background}

Schools in this triad were located in West Yorkshire. The Originator school and Partner school 1 served mainly white, lower middle and working class catchment areas in former mill towns. There were small proportions of pupils from Asian backgrounds, the numbers of children eligible for free school meals were around the national average, and slightly above average proportions of pupils had special educational needs. Partner school 2, meanwhile, served a more mixed rural catchment, including a middle class commuter area together with a large local authority housing estate. In the Originator school, performance in national curriculum tests at Key Stage 2 was well above the average: there had been a narrowing gender gap in English, within an upward trajectory of performance for both girls and boys. Key Stage 2 results in Partner school 1 were also above the national average, while results in Partner school 2, although broadly in line with the average, had seen a downward trend since 1999.

\section{The strategy: peer support through a paired reading scheme}

Peer support through a paired reading programme was well established in the Originator school, and was thought to contribute to that school's success, and all three schools were familiar with the paired reading programme published by the local education authority. There was concern in all the schools about small groups of under-achieving boys, and the belief that a common factor among such boys was their lack of self-esteem in learning. The purpose of the paired reading scheme was not, therefore, primarily to improve reading, as was the case in the triad discussed in chapter 4, but rather to address some of the socio-cultural issues which were felt to inhibit academic success. The scheme was attractive, too, because it required few resources, and was relatively straightforward to put in place.

Although the idea came from the Originator school, the strategy was initially trialled over a six-week period with a small group of year 5 and year 3 boys. The older boys received intensive training in being a listener, and were given a reference manual and appropriate resources. Paired reading sessions, which included older boys sharing books of their choosing with younger boys, younger boys taking books to read aloud, and the reading together of wall displays, took place twice a week in lesson time. The year 5 boys 
completed a record sheet at the end of each session, giving marks out of five for their partner's reading, attitude, interest, degree of involvement and effort. They were encouraged to reward their partner with stickers, and wrote reports for parents at the end of the programme.

Following this trial, all three schools used a similar format for all year 5 and all year 3 children, and the impact on focus groups of under-achieving boys was monitored. A unique aspect in the Originator school involved year 5 children preparing a reading pack including a number of activities with a literacy focus, such as puzzles, word searches and games, which they used towards the end of each shared reading session. The fact that the initiative lasted only six weeks meant that triad meetings regularly became useful opportunities for discussion of any problems which had emerged, as well as new ways of developing the scheme. The scheme was evaluated by the project team again in the following year, when it was used with same cohorts of pupils, by then in years 6 and 4 .

\section{Assessing progress}

Interviews with the focus group boys at the start and end of the paired reading schemes identified a number of positive impacts. Boys' fears of being unable to help their partners quickly disappeared as they found they could share their knowledge with the younger pupils to the extent that progress was made. They also developed a keen sense of responsibility, taking their role as listeners very seriously:

You had to really concentrate. You couldn't look around or anything in case they got stuck on a word.

Although not all partnerships worked well (particularly those of mixed sex), most were able to negotiate appropriate reading materials, and felt they dealt successfully with partners who initially refused to cooperate. Indeed, social skills improved to such an extent that behaviour in the playground improved, as older pupils 'looked out' for their younger partners at playtime. All the boys experienced pride and a sense of accomplishment through being looked up to by a younger pupil:

It makes you feel proud of yourself because you've helped someone younger than you to read.

Several felt they had become more confident, and that taking part in paired reading helped them to feel good about themselves. Even boys who had been ambivalent at the outset, and were uncertain as to whether they would be able to help a younger partner, could articulate a number of benefits. Questionnaires also showed that almost all had enjoyed the paired reading and thought their partner had enjoyed reading to them.

Staff interviews and the schools' own evaluations supported the boys' perspectives on the success of the scheme, and staff were impressed at the level of responsibility shown by the year 5 pupils and by the developing social skills. Some teachers felt the year 5 children had developed an awareness of how people learn, and were able to apply the range of skills learnt in paired reading to their own learning, and several also noticed a more positive attitude towards school in some pupils. In Partner school 2, a self-esteem questionnaire showed the scheme to be particular successful for the focus group boys, where self-esteem scores increased to a greater extent than in the cohort as a whole. 
However, although interviews with staff and pupils indicate a similar level of change in all three schools, value added data show that there were differences, with Partner schools 1 and 2 showing the greatest level of progress between key stages 1 and 2 (Table 9.3).

Table 9.3: Key Stage 1 to Key Stage 2 Attainment for West Yorkshire Focus Group Boys

\begin{tabular}{|l|l|l|l|}
\hline Value Added Measure & Originator School & Partner 1 & Partner 2 \\
\hline Whole school & 99.2 & 101.6 & 100.3 \\
\hline Focus group boys & 98.7 & 103.2 & 102.3 \\
\hline
\end{tabular}

In the Originator school, however, the value added scores for the focus group boys were a little lower than for the cohort as a whole. This difference might be explained by the fact that the Partner schools both took on the strategy whole-heartedly, while the Originator school, although willing to share ideas, did not in fact undertake paired reading so explicitly once the boys were in year 6 due to staffing problems.

The broader socio-cultural emphasis on paired reading, to offer peer support and to develop self-esteem, had a number of very positive benefits, therefore, particularly in contexts when:

- the purpose of paired reading was explained clearly to older and younger pupils

- pairs of pupils knew what was expected of them at each session

- older pupils received proper training so that they knew how to run the session, how to apply relevant strategies and how to deal with off-task behaviour

- older pupils were set up as experts and role models

- same-sex pairings were used

\section{Evidence of changing attitudes across the triads}

An attitude survey, based on that administered by MacBeath (2001) was undertaken across all the schools taking part in the Project, and pupils' responses were assessed on four attitudinal scales: engagement with school, relationships with peers, self-esteem and behaviour. Pupils completed the survey at the beginning of year 5 and again at the end of year 6 . Results from the surveys for all pupils $[\mathrm{n}=1254]$ showed that between years 5 and 6:

- engagement with school declines;

- pupils become more positive about their relationships with peers;

- self-esteem remains broadly unchanged;

- pupils' perceptions of behaviour remain the same.

The finding that engagement with school declines was not unexpected, given that the survey was completed just after pupils had taken their key stage 2 national curriculum tests. It is likely, too, that they were disengaging with primary school as they prepared to leave and move on to secondary school; perhaps, too, pupils were apprehensive about 
going to secondary school, and this may have impacted upon their perceptions of school. These factors might also have affected the almost significant decline in self-esteem.

Compared to pupils overall, at the beginning of year 5 the profiles of the focus group boys from across all schools fitted their teachers' perceptions of them as under-achievers: they were less engaged, had poorer perceptions of their behaviour than other children and had lower levels of self-esteem. They were as positive about their relationships with their peers as other pupils, however, suggesting that some might have enjoyed the status of 'key leaders': indeed, in the West Midlands triad, some of the focus group were identified on these grounds. Without intervention it might have been expected that all four measures would have worsened for under-achievers relative to other pupils between years 5 and 6 . In actuality, the gap between the focus group boys and other pupils narrowed on each of the four measures, indicating that the RBA project had some success in its impact on underachieving boys. Furthermore, between years 5 and 6 self-esteem among the focus group boys actually increased, providing strong evidence that the project was effective in raising self-esteem for under-achievers.

The aim of the three triads following socio-cultural strategies was specifically to change pupils' attitudes to school, and to raise self-esteem for learning, and therefore the results of the attitude survey are particularly pertinent to these triads. Since one data set was lost in the post, data are available for only eight of the nine schools, and these show similar findings to those outlined above in terms of their relationships with peers and behaviour. The findings relating to engagement and self-esteem, however, are particularly encouraging:

- while engagement with school declines in schools as a whole, there is no change in 6 of the 8 schools, either in the whole cohorts, or in the focus group boys; in a $7^{\text {th }}$ school, while engagement falls among all pupils, it stays the same for the focus group boys;

- despite their identification as 'under-achievers', only in 2 schools were focus group pupils less engaged than other pupils at the end of year 6;

- self-esteem among all pupils remains the same, though it improves slightly in one school; among focus group boys it increases, and is only slightly lower than that of other pupils at the end of year 6 .

These findings suggest that the socio-cultural strategies were particularly effective in achieving what they set out to do: pupils in these schools on average appeared more engaged in school at the end of year 6 than in the project schools as a whole and selfesteem was, on average, higher. Further, evidence does point to the fact that attitudes to school among the under-achieving boys were positively influenced by the strategies in place.

\section{Conclusions}

It is, as already indicated, difficult to isolate any one set of strategies and hold them responsible for raising boys' achievement. Nevertheless, the case studies discussed above do indicate the success of these socio-cultural strategies in engendering involvement and engagement in school for groups of boys felt at the beginning of year 5 to be underachieving. By the end of year 6 , in most schools, the focus group boys achieved at the 
level predicted by their key stage 1 results, or at a higher level, when on the basis of their year 5 performance they would have been expected to do less well. Thus each of the three strategies pursued through these three triads does appear to some extent to address the conflicts between the values of school and community which sometimes lead to a lack of engagement and involvement in school, and lower self-esteem in school based on values which are hostile to learning. Where such conflicts are acknowledged and addressed, then, as we have shown, the outcomes can be very positive.

However, as with other strategies, the context in which initiatives are developed is crucial to their success. Though they can be quite different in their approach, as the case studies discussed above indicate, socio-cultural strategies are most effective if the following are in place:

- a focus on individual pupils and a commitment to address under-achievement at an individual level;

- imaginative thinking about how to engage individual pupils in the life of the school, so that no child is allowed to 'opt out';

- a willingness to use ordinary activities (such as circle time or school plays) in which all pupils are involved, to target and monitor individual participation;

- a willingness to take risks - through giving pupils responsibilities or special opportunities and supporting them to ensure successful completion - and through providing pupils with an appropriate framework in which they can make choices;

- keeping records to ensure equal participation - so that it is not always the confident, high profile pupils who are chosen for particular roles;

- strong leadership and a commitment across all staff to putting the strategies into practice;

- a sense of community and teamwork, alongside a culture of equality 


\section{CHAPTER 10: Gender and Achievement in Special Schools}

\section{Introduction}

The project's work in special schools allowed us to consider some of the connections between patterns of boys' and girls' achievement in primary and secondary schools and the learning experiences of boys and girls identified as having special educational needs. There has long been acknowledgement of an apparent over-representation of boys with special educational needs. In primary and secondary schools, for example, the incidence of SEN pupils without statements is nearly twice that for boys (around 1 in every 5 boys) than it is for girls (about 1 in every 9 girls). Similarly, the incidence of pupils with statements of SEN is much higher for boys than it is for girls. In January 2004, over 106,600 boys in primary and secondary schools had a statement of SEN (around 28 in every 1000 boys) compared with 41,400 girls (around 11 in 1000 girls), the former rate being over twice the latter (DfES, 2004e).

The reasons for this relate in part to issues such as boys' classroom behaviour often being more disruptive than girls', resulting in earlier referral to support services. Gender and ethnicity are acknowledged as key factors in the identification of pupils with emotional and behavioural difficulties for example (Evans et al, 2004). However gender in itself does not explain why individual pupils may be identified as having special educational needs. The assessment of pupils' special educational needs involves a multi-professional, individualised process of decision-making, taking parental and pupil wishes into account (DfES, 2001). The point at which special educational provision is made for individual pupils, and the nature of this provision, varies between schools and LEAs depending on current practice in that context, local assessment criteria and financial constraints. The result is a complex, differentiated system of special classes, units, schools and support services in each local area in which gender balances in number and achievement change to some extent from year to year partly in response to changing local circumstances and procedures.

It has been common since the 1978 Warnock Report and the 1981 Education Act to see the identification of special educational needs as placing pupils on a continuum relative to their peers rather than in a separate category. As a consequence, special schools include a very mixed population of pupils, commonly with small class numbers and marked individual differences. Special school teachers attend closely to pupils as individuals, drawing on detailed knowledge of each pupil's pattern of difficulties in learning and behaviour often linked to a combination of sensory, physical, medical and social factors. This individualised approach makes it difficult to analyse broad gender trends and patterns of attainment. However, special schools are not divorced from mainstream education and the wider world. Socio-cultural beliefs and pedagogic practices inevitably filter between special and mainstream schools. Parents, teachers and other school staff have perspectives and expectations that cross the boundaries. Pupils may be on inclusion programmes with local mainstream schools and they may have friends, club activities and jobs in the local area. The conclusion is that while questions about gender and achievement in special schools are highly individual and contextualised, they also have connections to the issues 
discussed in other chapters of this report with regard to specific teaching and learning strategies as well as the general processes of implementing change in any school setting.

\section{The Research Participants and the Focus of Inquiry}

The research in special schools could not begin in the same way as the mainstream project, that is, by identifying schools already 'narrowing the gender gap' by using specific strategies to raise boys' achievement. At the outset, it was not clear whether a 'gender gap' existed in special schools, or what its nature might be, and one of the first research tasks was to formulate appropriate research questions for the special school context. This need to identify questions about boys' achievement quickly led to the realisation that a key factor lies in the data that are used to measure the progress of pupils with special educational needs. The first school invited to take part had been recommended on the grounds of their well-established use of the $\mathrm{P}$ Scales to monitor pupils' progress in working towards Level 1 of the National Curriculum. Other special schools were then identified for their geographical proximity to the first school or to mainstream clusters already involved in the project.

The initial research group involved six special schools, three for pupils with more severe, profound and multiple learning difficulties and three for pupils with moderate learning difficulties (although all the schools included a wide mix of pupils with learning, behavioural, sensory and physical difficulties). Two schools were unable to continue for the whole project, leaving us with four schools: one for pupils with severe, profound and multiple difficulties, and three for pupils with moderate learning difficulties. Two schools were in rural East Anglia, one school was in the West Midlands conurbation, and one school was in an expanded new town community in Eastern England. The geographical distances and the differences in pupil population meant that it was not possible to identify school triads in the same way as in the intervention stage of the mainstream project. The schools worked individually on their own inquiries with the support of the research team. However, discussion and collaboration between schools was seen from the start to be a major element of the research project, and the links between the special schools and some primary and secondary schools in the mainstream project were developed in the context of Cambridge-based conferences.

The four special schools identified target groups of boys (and girls) and focused variously on such factors as self-esteem, motivation, writing progress, reflective talk, learning styles, independent learning, staff and pupil expectations, and whole-school development. The three schools for pupils with moderate learning difficulties developed interventions for pupils in Year 9, 10 and 11. The school for pupils with severe, profound and multiple difficulties focused on younger children in Key Stage 1.

\section{Gender, Achievement and Special Education: some general findings}

There is an identifiable but not extensive field of research connecting gender with disability, learning difficulty and special education. Some work draws explicitly on sociocultural understandings of gender. For example, McDonagh (2000) demonstrates how historical studies of literary representations of men and women with learning disabilities have indicated contrasting emphases on either diminished male incapability or disruptive 
female sexuality. Benjamin (2003) examines the connections between social constructions of masculinity and femininity and constructions of special educational needs, asserting that 'SEN is a gendered phenomenon' (p. 247) which must be understood as such in developing inclusion.

Biological accounts of boys' developmental difficulties are also found in the literature. For example, Skårbrevik (2002), writing in the Norwegian context, suggests the need to take into account the phase of education in examining gender differences and SEN, proposing that genetic or biological differences come to the fore in the pre-school years while later identifications depend more on social factors and pedagogical mismatches. There is a widespread view that boys are generally more vulnerable to adverse biological experiences from infancy, including genetic syndromes like Fragile X (Freides, 2001), and many more boys than girls fall into categories or syndromes associated with SEN such as autistic spectrum disorders. Gender has been put forward as a key factor in children's resilience over time, although evidence now suggests the existence of gender differences in responses to childhood adversity rather than, as previously thought, a protective or compensatory effect of being female rather than male (Fergusson and Horwood, 2003).

One of the main problems in reviewing research evidence in this field is the difficulty in gathering reliable and valid evidence of overall gender differences in attainment, inclusion and special provision. It should be noted that any use of quantitative data in this field raises significant technical issues about categorisation, measurement, completeness and accuracy (Florian et al, 2004). However, research has suggested that gender variations do exist between schools in the ways that special educational needs (SEN) budgets are allocated and in the implementation of SEN procedures and support (Daniels et al, 2000; Vardill and Calvert, 2000; Hill, 1994). Croll and Moses (2000) note that the overall gender ratio (more boys than girls) for pupils in Key Stage 2 (7-11 years old) identified with SEN did not change substantially between 1981 and 1998 in spite of growing concerns about 'male under-achievement' in the 1990s. They did, however, find an increasing proportion of boys regarded as having emotional and behavioural difficulties in that time.

In general boys are much more likely to be regarded by teachers as having SEN, but this does not seem to be a simple matter of gender stereotyping and categorisation. There are strong, complex connections between pupils' classroom behaviour, learning and achievement, and Cline and Ertubey (1997) found that giving teachers more contextual information helps to reduce the impact of gender on teachers' judgements about individual children. The interactions between different areas of SEN are evident. For example, different rates of SEN referral for learning difficulties can arise via observed differences in classroom behaviour which are exacerbated by peer pressure, cultural clashes and psychological processes of motivation and 'self-worth protection' (Jackson, 2002). A consequence of the acknowledged difficulties with 'boys' behaviour' in schools can be that while girls may be numerically under-represented in special schools, those that are identified as having learning or behaviour difficulties are likely to have more severe problems than the boys in the same setting. They may even have their special needs as young women ignored altogether (Malcolm and Haddock, 1992). From the boys' point of view, the problem can be one of undue assumptions about learning difficulties on the basis of difficult classroom behaviour. Daniels et al (2000) found that boys are given proportionally more and higher status SEN support in mainstream settings, but they may 
not receive support clearly matched to their needs (e.g. being given additional reading instruction as a response to inappropriate behaviour). This points towards a need for further research with a gender perspective on teaching and learning processes for pupils receiving special support, an area not well covered in the literature.

In addition to the conceptual difficulties of carrying out research in the area of gender and special educational needs, there are some significant methodological and ethical issues to be taken into account. These relate, for example, to the small numbers, the problems in communicating with pupils with certain types of SEN, and the difficulties which can arise in gaining informed consent in the special school context. There are also questions about the role of research in responding to hidden agendas and wish fulfilment for pupils, parents and teachers who face considerable daily challenges in teaching and learning. With regard to this last point, it is important to emphasise that the relatively hidden issues of gender in the field of special education can be of central personal and lifelong importance for the pupils involved. In special schools there can be significant problems for male and female pupils in developing gender identity, experiencing relationships and benefiting from sex education - particularly for pupils with the most severe learning, physical and emotional difficulties. There can also be long-term implications for men and women with learning difficulties in their post-school experiences, relationships, learning and employment (Riddell et al, 2001).

\section{Developing Research Questions}

At the start of the project, special schools representatives came together to discuss questions about gender in special education and consider what their own research questions might be. There was a consensus among them that the gender differences which are apparent in mainstream schools are less obvious and clearcut in special schools. Here, small classes and the strong emphasis on addressing pupils' explicitly defined special needs, through an extensive use of multi-sensory and practical teachings approaches, tend to move the focus away from issues of gender or ethnicity, towards meeting the specific needs of the individual. This being the case, their combined list of ideas about some key areas for research and development was as follows:

- refining and extending ways of measuring progress, both quantitatively (e.g. P Scales) and qualitatively (e.g. techniques for interviewing children with communication difficulties);

- specific gender-related issues (e.g. gender identity; physical care; particular conditions; sexuality and personal relationships). Note also additional cultural issues in these areas;

- issues arising from the over-representation of boys in special schools (e.g. one girl in a class of boys);

- issues common to special and mainstream schools, but exacerbated for the boys in proportionally high numbers transferring from mainstream (e.g. sense of failure, low self-esteem).

The four participating schools were all concerned in some way with gathering data on pupils' progress, and common special/mainstream issues were also central to the research in three of the schools, where the focus was on different aspects of boys' writing, selfesteem, expectations and engagement in learning in Years 9-11. The pupils involved in 
these projects included some who had transferred from mainstream schools into Year 7 and some concurrently involved in inclusion programmes with neighbouring secondary schools. The fourth school also focused on issues common to special and mainstream schools in looking at the learning needs of primary pupils identified on the autistic spectrum, specifically the effectiveness of 'work stations' and visual aids to promote concentration and independent learning. The pupil group involved in this research had rather severe and complex needs, but pupils with similar types of difficulty can be found throughout the mainstream and special system. The teaching approach adopted can be compared with the mainstream projects on responding to pupils' learning styles and preferences (see chapter 5). Social integration and personal relationships were also seen as key factors in each school's attempts to raise pupils' achievement.

From the special school perspective, involvement in the research required a willingness to ask whether enough of the right sort of work was being done to raise boys' (and girls') achievement in a setting where it could be argued that the whole focus is on supporting pupils whose achievement is low compared to their peers. Asking about boys' achievement could therefore be seen as a rather radical question about gender bias in special schools, which are commonly perceived as environments where boys and girls are somewhat detached and protected from the ordinary social pressures.

\section{Case Examples of School-Based Inquiries}

There was much diversity in the schools' interpretations of the research focus and their approaches to their own investigations - highly influenced by the specific features, interests, priorities and needs of each school context.

\section{Case Study 1: Teaching and learning English in Years 10 and 11 in the wider context of whole school development}

Initial discussions with staff at this school helped to identify an area of interest in pupils' writing, particularly in their understanding of writing and what they perceived as 'good writing'. Interviews were carried out with pupils in Years 5, 8 and 10, which suggested that many pupils tended to focus on the technical aspects of writing as the basis for evaluation, commonly emphasising neatness rather than content. However, the interviews also showed that pupils recognised that they were involved in writing across the curriculum and outside school, which was promising for developing their motivation and learning in all aspects of writing in the future. The research was taken forward with the aim of investigating the question:

What teaching approaches support improvement in boys' writing?

There were two classes involved over two years. The pupils involved were described as having a range of emotional, health and learning difficulties and at the beginning of the first year of the project in Year 10 none of them was achieving higher than Level 2 to 3 in English. Most of the pupils took a cautious approach to writing, unadventurous with vocabulary or spelling in case they made mistakes. The class teacher involved took an integrated approach to teaching English, including careful scaffolding of tasks over a period of several weeks leading to a specific genre outcome. She catered for diversity in the pupils' learning preferences by using visual and aural approaches, making learning objectives explicit and aiming to help pupils develop self-evaluation strategies. The 
research did not involve changing or adding to the teacher's approach. Attention was given primarily to close monitoring of the pupils' responses to teaching, particularly their increasing capacity to write confidently, to talk about their writing and to understand that writing is meant to reflect their own thoughts, ideas and feelings. The project also involved the production of a professional development video, 'Scaffolding Writing', analysing the teaching strategy and the pupils' progress in reflecting on their learning.

At three points in the year samples of writing were analysed, and the pupils were interviewed about their attitudes to writing and the processes of their learning. In the first year it was found that all of the pupils had made significant moves in their approach to writing, their attainment levels, their independence, pride and assurance as writers and their abilities to talk about their knowledge of the writing process. Assessments at the end of Year 10 showed that four of the six boys involved had moved at least two NC sub-levels during the year and one pupil had moved a whole writing level. This contrasts sharply with the expected timescale of two years for this rate of progress. Analysis of the writing showed developments in overall fluency and assurance, the technicalities of spelling and punctuation, and the range of different types of text tackled. The teaching strategy had also helped the pupils to work collaboratively. This progress continued as the pupils went into Year 11. Periodic reviews indicated that all the pupils continued to comment on the content and meaning of their writing, and they talked about the importance of planning, taking notes and reading through their work carefully. Teacher assessment at the end of the year showed that two of the boys had maintained the gains made during the previous year and that four of them had made a third or two thirds of a level more progress.

In the second year, however, the new Year 10 group did not replicate the previous year's experience of greater control over writing or their progress in developing a metalanguage to talk about writing. The pupils remained preoccupied with the surface features of handwriting and spelling, and the discernible progress was only in terms of handwriting and control of sentences and paragraphs. The particular special needs of these pupils and the relationships within the group seemed to hinder the boys' progress, raising a question about whether young writers need to have a sense of personal control to underpin their control of writing. Despite the teacher's efforts, the boys in the second cohort did not move very far towards greater independence as learners or writers, although importantly, the girls made more significant gains both in assurance and independence and in writing levels - pointing towards the need to look for distinctive features in this second group of boys.

While the Year 10/11 writing project was developing, there were also significant whole school developments prompted by the initial broad research question about how to understand and respond to gender differences in attainment in the special school context. The school had been the first special school to be involved in the Raising Boys' Achievement project because of the progress already made in using the $\mathrm{P}$ Scales in assessment and target setting. The integration of the research focus with other initiatives in school was prompted and facilitated by the deputy headteacher's increasing whole-school responsibilities for professional development, curriculum and assessment alongside her role as co-ordinator of the school's research activity.

Several different strands of activity emerged for the deputy headteacher as the project continued: 
- her involvement in implementing the school improvement plan, performance management and school self-evaluation

- her participation in NPQH training courses

- her role in planning professional development for staff

- her involvement in refining the uses of the P Scales in school and developing software for data management

- her responsibility for writing the Learning and Teaching Policy

Each of these activities contributed in some way to gaining a deeper understanding of how attention to gender fits into the network of activities aimed at understanding and improving learning and teaching for all pupils in school. For example, the development by the staff of an agreed approach to lesson planning drew attention to what were seen as key features of learning and teaching including: attention to pupils' learning styles; clear objectives; giving time to think; reinforcing new learning; providing activities to promote learning; and a plenary to review and look towards the next lesson. Some of these relate to pedagogic strategies thought to help many boys and some are more generic. However the focus here is not on the strategies themselves as interventions. The lesson plan was seen by the deputy headteacher to be facilitating rather than directive for teachers, and she believed that its successful use depended on teachers' understanding of the underlying principles supported by their participation in discussion about the lesson planning approach adopted.

This understanding of the importance of attending to teacher's knowledge about factors affecting pupils' learning is backed up by the results of a set of interviews with the teachers and learning support assistants towards the end of the project. On being asked whether gender is relevant in school, most said 'yes'. However they gave many different reasons and examples applying to different age groups and subjects, including opinions about traditional gender bias in some subjects such as design technology; observed preferences for pupils to work in single sex groups; girls 'taking over' in some practical activities; boys tending to be more independent workers but requiring more focusing and motivating than girls at Key Stage 4; boys being more competitive and finding it difficult to accept failure; boys being more active and physical, but slower than girls to engage in new experiences such as the sensory nature table. All the staff who felt that gender is relevant to teaching and learning could identify the strategies they use in response ranging from modelling, encouragement and class discussion to more direct and specific interventions such as regrouping pupils, targeting rewards, and using visual approaches and ICT.

The impression is that gender is embedded in the thinking of many special school staff, but that it is closely integrated with the other knowledge that guides each person's strategic decision making and action in school. It is only when this practical knowledge is targeted, analysed and shared that gender may emerge as a common concern appearing in different guises. The deputy headteacher remarked at the end that involvement in the Raising Boys' Achievement project had supported a wider and deeper view of gender in teaching and learning, and that the school was more able to take account of gender factors. Awareness had also been raised about ethnicity and other factors cross-cutting with gender with a view to demonstrating non-discrimination in school, indicating a more questioning approach to school policy and practice closely linked with the school's increasing facility 
in gathering relevant evidence about achievement, professional development, teamwork, 'good teaching', and understanding of pupils' perspectives.

\section{Case Study 2: Boys' self-esteem and engagement in learning}

This school was involved in the project under the guidance of the headteacher. The starting point was his observation that in the last few years the school had received a large influx into Year 7 of pupils (mainly boys) who might be described as 'not typically MLD' (moderate learning difficulties), but who were streetwise, disaffected and somewhat resentful of their special school placement when their primary friends had gone to local secondary schools. The focus of research was on the progress of a small group of boys in Year 9, all of whom showed notably good sporting prowess, but whose other academic attainment levels were not significantly different from other pupils in this school where approximately 20\% achieve levels 3-4 in English and 4-5 in maths and science at Key Stage 3. In Year 11, boys' attainment is at least equal to girls' and higher in some subjects. The focus group's attainment levels ranged between 2-4 in English, and between 3-4 in maths and science, having risen from 1-2 in English and 2-3 in maths and science in Year 7. Observations of the pupils' general behaviour and attitude to school, together with the assessment of the educational psychologist, supported the headteacher's view that low self-esteem and disaffection were significant factors for these pupils. The broad research question asked whether the current ethos of the school and the learning/teaching environment offered enough to effect a change in these pupils' attitudes and self-esteem so as to significantly change their academic and social achievement.

The research involved the headteacher in identifying current strategies, continuing support and evaluating progress, rather than introducing a specific intervention for the identified group of boys. Support strategies were identified at different levels:

- $\quad$ some directly with individual pupils (e.g. counselling);

- some embedded in the school practice (e.g. Good Work assemblies);

- some at the wider family and social context (e.g. parent liaison; appointment of School Welfare Officer).

The aims were variously directed at:

- behaviour management (e.g. rewards for appropriate behaviour);

- social relationships and role modelling (e.g. working with headteacher; buddy system);

- metacognitive understanding of learning aims and expectations (e.g. target setting and visible evidence of progress; clear expression of expectations to pupils);

- emotional support (e.g. counselling; calm, positive discussion of feelings);

- $\quad$ self-concept and motivation (e.g. sports activities).

Although the pupils' experiences of inclusion in local secondary schools for certain examination courses demonstrated both social and academic progress over time, there was continuing evidence of behavioural and attitudinal difficulties within the focus group. In the headteacher's view this was partly exacerbated by the difficulties of transfer from Year 9 to Year 10 (in terms of curriculum, class size and teaching style). For example, for five boys the total recorded behaviour incidents in Year 9 was 22, while in the first term of year 10 the total was already 19. Attendance of four of the pupils had dropped by about $10 \%$ 
compared to Year 9. From previous experience with that age-group the headteacher expected the pupils' confidence, behaviour and attitudes to improve as they saw evidence of their examination achievement in Year 11 - a key goal for pupils and teachers in Key Stage 4 in this school setting.

\section{Case Study 3: Expectations and inclusion}

This school focused on a group of pupils with similar needs to those identified in the previous case examples. The deputy headteacher found in a whole-school review of core subject attainment using P Scale data, that certain boys in the upper school (Year 9/10) emerged with a distinctive (comparatively high) profile. His hypothesis was that some pupils in school come to expect too little of themselves in academic terms in a context where they are necessarily provided with the emotional and social support required to avoid exclusion. At the time a small number of individual pupils were successfully joining the local secondary school for specific subjects (such as art) in an informally developed inclusion programme. The identification of this group of Year 9/10 pupils raised the possibility of developing a more active inclusion programme for a larger number of pupils. The key goal was to evaluate the effects of inclusion on raising pupils' expectations and achievement.

It was clear from the start that there would be a number of implications and requirements for developing the inclusion programme. These were seen to include not only the financial implications of implementing a more formal and extensive connection with local secondary schools, but also the motivation and justification for doing so. Inclusion might be justified on several grounds, but there would also be sticking points - including parents' views and preferences. It was seen as vital here to understand the pupils' own perceptions and opinions as well as those of other people. To this end, the Key Stage 4 co-ordinator took responsibility for introducing a more systematic discussion with pupils at regular interviews, using an 'attitude to school' questionnaire format. Some questions proved to be particularly revealing, as when a question about being encouraged at home to do well helped three pupils to begin to talk about their difficulties in this respect.

The development of the inclusion programme was tackled at different levels, including work with pupils, parents, local school contacts, resources and practical issues, and funding. As time went on improvements were seen in attainment (for example, 4 boys taking GCSE courses), pupils' self-esteem, staff-pupil relationships, home-school relationships, attitudes of other pupils, and raised staff expectations. Several of the pupils showed their enthusiasm with comments like:

When I walk in (secondary school) I feel a little bit nervous but I have the confidence as well to do it on my own.

The maths is all right. It makes made me feel good to be chosen.

I like harder work because it is a challenge.

Teachers are encouraging me more now.

There was growth in the local inclusion network of mainstream and special schools, and increased LEA funding. The school became interested in exploring further the two-way process of reintegrating pupils where appropriate and offering support to mainstream pupils who are at risk of becoming non-attenders. However, there were also costs to weigh 
up in considering the ongoing time investment, financial difficulties and practical demands of managing inclusion, with one of the main issues for future consideration being adequate staffing levels for teachers and learning support assistants to facilitate what is seen to be a good way forward in school.

\section{Case Study 4: Supporting concentration and independence in classroom learning}

This project had already been established in a school initiative supported financially by the LEA and led by a class teacher working on her own research with children in Key Stage 1. The intention was to carry out a systematic investigation of the effectiveness of using 'work stations' to engage pupils on the autistic spectrum in learning activities. Children with autistic spectrum disorders (of whom a high proportion are boys) typically find it difficult to organise information flexibly, to transfer their learning to new situations and to engage socially with other people. Imaginative play and other open-ended activities can be particularly problematic, and considerable teaching support is needed for them to profit from the worthwhile learning activities typically offered to young children.

The work station approach involved children in working under supervision on a series of familiar activities, using a visual timetable to organise the session and initially screened from the rest of the classroom to allow concentration for that period of time. The children were able to choose a reward on completion (such as time with a favourite toy). The aim was for the children eventually to transfer the process to working more independently in the classroom. The teacher who took responsibility for this project saw the approach as a way of responding to the learning styles of pupils with autism, building on their strengths and preferences (for example, for visual images, concrete materials and few social demands). She worked closely with the learning support assistant to observe and monitor the children's progress, including how much support was needed on each occasion. She also consulted with other teachers and helped them to implement the strategy in other classes. Strong overall evidence was found of increasing time on task and longer periods of independent work. There were also notable individual developments in vocalization, reading, motivation and behaviour. For example, one child quickly moved from a photographic schedule, to photographs and words, and then words only. Another child settled to work at the station, even while her other classroom behaviour continued to be 'self directed and nonconformist'. Other teachers became aware of the need to plan differently for children who showed that they could cope with higher level tasks at the work station than in the classroom group situation (indicating their sensitivities to the social demands of group interaction).

As is typical in special schools, not all the children showed the same rates of progress, and some found that factors like health difficulties were making it difficult to sustain the necessary continuity. However the teacher's general conclusions were that the work stations and visual schedules enabled pupils to develop new skills which sometimes transferred to the classroom allowing integration in the class group. There were also benefits for some parents in seeing evidence of what their children had learned on video and pursuing this at home. She could see the potential for developing this work with younger children in the nursery and in developing the methods to teach skills in groupwork. She also remarked on the applicability to mainstream education - including an example of a child who responded to a piece of tape on the desk to represent the screening 
and boundary effects of the work station with which he was familiar. However, she and the headteacher recognised the costs associated with this initiative, particularly in finding time for proper staff consultation and for gathering systematic evidence of progress through close observation.

\section{Some General Conclusions from the Case Studies}

Some key questions emerged from these intervention strategies which were developed and monitored within the four schools:

1) Does the learning-teaching environment and the ethos of special schools offer enough to develop positive attitudes, enhance pupil self-esteem and effect change in academic and social achievements, especially for boys (and girls) who are likely to have 'failed' in mainstream schools?

In Case Study 2, the perceived danger had been that pupils' experiences of failure in Year 6 and transfer to special school, compounded by difficulties in home life and early developmental experience, would produce low self-esteem and subsequent disaffection from school. The headteacher believed that in these circumstances a pupil would not perceive themselves as a 'whole, rounded person who had achieved to the best of their abilities.'

Factors such as staffing and curriculum balance are crucial longer-term issues for consideration. A key point here, though, is that the focus is on particular pupils in a specific school context, not general policies for pupils with SEN in general. It is evident from this work, not only that the evaluation of 'enough' has to be contextualised in the realities of school life, but that it is possible to judge success in measured way acknowledging what is done well and what to do next. This point was also evident in Case 4, where expert knowledge of 'what may work' for children with autistic spectrum disorders was tested systematically. Close attention to the progress of individual pupils using the work stations allowed a realistic and constructive understanding of how the strategy worked in practice, taking account of the variations in individual pupils' circumstances, needs and progress over several school terms.

2) How is the social and emotional support offered by special schools best accompanied by appropriately high expectations for academic and social behaviour, especially for vulnerable boys and girls who may find necessary protection in the special school setting?

This is the question that was at the heart of the inclusion programme in Case Study 3, and the issue of balancing social and emotional support with high academic expectations was evident in all the schools. The multi-level approach to planning was important in helping the staff working on inclusion to take account of multiple perspectives and concerns including those of the pupils, parents and staff - while keeping in mind the main aims of developing inclusion to help in decision making and setting priorities. It was clearly necessary to take a long-term, multi-level view in order to implement and maintain change in school, while also being sensitive to the interests and feelings of those involved (particularly parents in this case). 
In the end the question points to how we understand 'achievement' in broad terms, and how that relates to the balance of support and expectation. The issue becomes crucial for pupils in Key Stage 4 looking towards life after school. The headteacher in Case Study 2 held a wide view of 'achievement'. In talking about his pupils' progress he made a point of noting their involvement in football training, in organising tournaments in 5-a-side football and pool, and in unprompted 'acts of kindness', such as showing empathy to and comforting a bereaved pupil. The implication of the practical use of this package of support strategies is that social, emotional and academic development are closely connected - an argument strongly supported in current psychological thinking.

The multi-level perspective and broad view of 'achievement' is clearly important in balancing social, emotional and academic support and expectation. However establishing the security and achievement of pupils must be weighed up against their day-to-day experiences of scrutiny and surveillance in the special school setting. Special schools are often small scale enterprises compared to other schools, with many staff around all the time. The close monitoring that helps many pupils to learn in mainstream schools (because it allows pupils to save face with their peers about making an effort to work), is likely to be experienced differently by pupils for whom close monitoring in a previous school experience resulted in detailed SEN assessment and ultimately special school placement. The perceived value of monitoring achievement very broadly needs to be examined in the light of pupils' actual experiences and their views of what it means for them.

3) How can initiatives be maintained and developed over time, given the cohort changes from class to class and year to year in special schools? What are the implications for teaching and learning policies, curriculum development and teachers' professional knowledge?

All the schools were concerned with maintaining initiatives, and teachers in Case Studies 3 and 4 could see serious problems arising if additional costs were not met. However sustaining an initiative through time presents even more subtle challenges in teaching, learning, curriculum development and professional development. For example, in the writing project in Case 1, the two year groups differed not only in levels of achievement but also in their capacity to use a metalanguage to talk about the content and intentions of their writing. Where the boys in the first group noticeably grew in confidence and a sense of self-efficacy, most of the boys in the second group failed to thrive in these respects. The girls' improvements, however, indicate that it is likely that there were no great differences in the teaching approaches used, but that the nature of the learners and their needs is a key factor in success. This is a strong indicator of the 'specialness' of special education. Whilst some of the successful approaches used in mainstream education can be equally effective in both types of school, the special nature of the learners themselves means that initiatives to improve boys' writing need to take account of individual learning needs. A conclusion could be that the familiar mainstream strategy of focusing on the meaning of writing as the motivation for teaching and learning may have to be put aside where pupils' technical difficulties are overwhelming and 'success' has to be evaluated individually (at least in the short term). However the broad curricular aims for all pupils are not then abandoned - not least because some pupils in special schools could equally be in mainstream, and vice versa. 
The findings in Case Study 1 provide evidence of the complex ways in which initiatives may become embedded in school practice so that teachers can consider their pupils' progress within a broader context. The question of teachers' professional knowledge and expertise was high in the deputy headteacher's mind in all her school development activities. She remarked at the end of the project that part of her role was to help staff become aware of all the different projects happening in school, using staff meetings or professional development time for teachers to speak about their work. This can be seen as supportive of sustaining change in school by providing the 'drip, drip, drip' of information and encouragement to the staff team, without necessarily knowing in advance how a project will develop. The idea is to share good practice within school, and the deputy headteacher saw her own involvement in the research, including her interviews and conference attendance, as a key contribution to her overall role in whole school development.

\section{4) How can useful, reliable and valid evidence be gathered about the broadening academic and social achievement of pupils with special educational needs, given the small samples and wide individual variability between pupils?}

The issue of measuring attainment in special schools loomed large in our minds from the start of the project, that is, how it is possible to measure, compare and evaluate the progress made by individuals and groups of pupils with SEN who are not necessarily following the standard route of assessment in national tests and public examinations. The quantitative use of curriculum-related measures of attainment like the P Scales was central to early discussions with the special schools. However it became clear that managing this performance data could present problems for schools. For example, in Case Study 1 the deputy headteacher identified nine different uses of the P Scales, ranging from feedback to teachers, target setting and parental reports to school improvement planning and accountability. One of the main problems for her lay in deciding how to organise the data to allow these various outputs while also incorporating the qualitative understanding that allows understanding of what it means for pupils, teachers, parents and other agencies.

As time went on all the schools became more concerned with gaining a wider picture of pupils' progress in school. Many different techniques were used to collect research data, supporting a broad view of 'achievement'. These included attitudinal questionnaires, analysis of pupils' writing, classroom observation and video recordings, attendance monitoring, meeting notes, behaviour incident records, collection and analysis of P Scale data and individual and group interviews.

In some contexts one approach to data collection was central to the project, such as the systematic observations in Case Study 4. However in general, the range of data gathering approaches gives an indication that to look at 'achievement' is to look carefully at several different perspectives of the pupil in several different contexts. This supports the view that achievement and progress are the results of collaboration between pupils, teachers and other people in contact, not just reflections of pupils' personal effort and ability. Indeed, the headteacher in Case Study 2 reflected at the end of the study that the research reinforced his beliefs about the importance of establishing a relationship with children in order to affect anything in their lives and learning - suggesting that efforts to raise achievement centrally involve this relationship and not just pupils' own efforts and capabilities. 


\section{Final Thoughts}

The findings from the special school projects suggest that a gender impact on the achievement profile in special schools is almost inevitable given the social and biological factors involved, but it is complex, interactive and not easily discernible. It changes from year to year and it occurs for a number of different reasons. However, a simple 'gender' distinction fails to tell the whole story, especially when combined with the slippery definitions of special educational needs and a concern for social justice. As Daniels et al (2000: 64) remark:

'...categories are analytical tools with which we understand the social processes we seek to monitor and ultimately change... We know that boys and girls are not treated in the same way. We do not know whether this is fair. It may well be that we should seek to establish new forms of difference rather than impose sameness'.

The question of sameness and difference is central to the discussion of special school practices. Without attempting to generalise too widely, it seems clear from our case examples in Key Stages 3 and 4 that while boys with learning and behavioural difficulties in special schools are in some danger of losing confidence and momentum in their learning, there are useful steps that can be taken over a period of time. As also seen in Case 4, approaches to teaching can match those found to be helpful in mainstream schools, but the variability of pupils' individual needs and the intrinsic nature of certain areas or types of SEN adds a significant layer to the special school teachers' knowledge and decision-making. There may also be certain contextual factors relating specifically to special schools. New curriculum developments set challenges as well as opportunities (e.g. 14-19 changes and alternative curriculum initiatives). The current inclusion strategy calls on special school staff to make links beyond the school while also contributing to their own school as a learning organisation. Special school teachers also have specific concerns about the content of initial teacher training and new teachers' skills with pupils with special educational needs.

It is not clear, however, that this results in an entirely separate set of 'special school' issues or practices. This conclusion matches other research findings which provide little evidence for a separate special education pedagogy and suggests that the priority is to use special education knowledge to develop inclusive pedagogy in the current policy context (Davis and Florian, 2004). The research presented here suggests that understanding gender factors in combination with special educational needs can apply to pupils in any educational setting, and deciding to examine these can have unexpected, challenging and rewarding results for all involved.

\section{Some key conclusions emerge about the value of:}

\section{Being willing to ask challenging questions in school}

'Special educational needs' are complex and multifaceted in nature, varying also as a function of gender, ethnicity, cultural practices, social class and the specific school contexts of teaching and learning. It can seem difficult or apparently irrelevant for special school teachers to focus directly on gender when the pupil population in most special schools is so diverse, but a willingness to do so can produce valuable insights when close links are established with teachers' professional concerns, aims and practice. This is the value of 'driving from the bottom'. 
Using a broad concept of 'achievement' with equally broad approaches to gathering data

It is essential to work with a broad, interactive and contextualised understanding of 'achievement' and use an equally broad range of qualitative and quantitative approaches to investigation and measurement - weighing specific performance data against the broadening achievement of individuals. This close attention to pupils' individual achievements and differences also helps to provide a better understanding of the complex processes operating for all pupils in school.

\section{Using combined, multilevel strategies for intervention}

Interventions are likely to involve combinations of identifiable strategies, with implications at different levels of the school system and beyond. Strategies directed at transfer between different subjects and contexts may be of particular relevance for pupils in special schools.

\section{Planning for maintenance while acknowledging the likelihood of uneven progress}

The maintenance of most new initiatives requires an investment of time and energy with planning and support in staffing, whole-school development and relationships with pupils and parents. There are likely to be phases of development, often starting with very small steps of progress. It is necessary to integrate new strategies with current interests, policies and priorities, acknowledging the likely impact of pupil cohort changes from year to year.

Making explicit connections between mainstream and special school practices and procedures

Gender may help to explain in general terms why some pupils enter special schools. However gender in itself does not explain why individual pupils enter special education. Some of the boys who achieve less well in mainstream schools will enter special education, but not all of them. This points to the need for further research on the SEN decision making processes with explicit reference to the effectiveness of strategies used for raising boys' achievement in mainstream schools.

Extending collaboration and shared professional development between special and mainstream school colleagues

Boys are also boys in special schools, often with full social lives outside school hours. Certain 'mainstream' social and educational influences and practices apply to them, and certain special school teaching strategies seem to match and perhaps extend the approaches for raising achievement being developed in mainstream schools. This is a case for extending the special-mainstream collaboration and shared professional development already in place in many LEAs. 


\section{CHAPTER 11: The Broader Concerns: Developing, Supporting and Sharing Innovative Ideas}

Previous chapters of this report have focused on the context for the Raising Boys' Achievement Project, discussion of research design and approach, and the various strategies which were implemented and evaluated. In this chapter, we reflect on broader concerns to do with supporting and sharing innovative ideas within and across schools.

\section{Within Schools}

\section{Understanding the context and meeting the preconditions for development}

A recurring theme in the preceding chapters, whether related to pedagogy, forms of organisation such as single-sex groupings, strategies which focus on the individual, or socio-cultural approaches, is the fundamental importance of context. Context is important at both school and community level. We are confident that all the strategies outlined above have the potential to be successful, but would emphasise that there can be no 'onesize-fits-all' approach: a national strategy to raise improvement must have that as its aim, but that aim can be reached, we suggest, by different routes. This report, therefore, does not advocate one specific approach which might be implemented in any school and in turn lead to improved academic achievement. It suggests, instead, alternative ways forward, some of which might be appropriate to one particular context, and some to another.

Where strategies have been successful in terms of changing attitudes and achievement, it is because schools decided they were appropriate for their particular circumstances, and because they chose to take them on board. As indicated above, the research team worked with the schools through collaboration and not imposition, and schools adapted strategies in the light of the cultural contexts of their own environments. Thus in some areas (such as the inner city triad in North West England, discussed in Chapter 8), where the conflicts between the ethos of the school and that of the outside world are particularly evident, a socio-cultural approach was adopted. The purpose was to create a more cohesive school community within which learning could take place. In other environments, particularly at primary school level, it might be the case that the problem is less one of boys who are disengaged with the ethos of the school, but more an issue of them lagging behind girls in terms of literacy. In one of the primary triads discussed in Chapter 4, for example, in contrast with national data, boys achieved less well in reading than in writing at key stages 1 and 2. This led to an initial emphasis on reading, the involvement of homes and families, and a strategy which sought to raise the profile of reading as a pleasurable activity.

These two examples suggest the need for a heightened awareness of local issues and a willingness to take risks in developing innovative approaches specific to each local context. Thus one of the important outcomes in the development of the RBA project in each school was greater understanding of what was being done to raise achievement. This was not always easy: in the pilot stage of the project, the schools we selected did not always know why the gender gap in their schools had closed. Certainly all the schools had put into effect certain measures to raise improvement, but these were not necessarily 
gender specific. Furthermore, no school had focused on just one form of intervention. We found at the pilot stage, and again as we entered the main phase of the project, that schools tended to employ a basket of strategies, particularly primary schools. Sometimes the basket contained explicit, clearly articulated strategies, but more often it contained approaches that had become such an implicit part of the culture of a particular school, that the school found it difficult to acknowledge that is was doing anything special. An important task for each linked researcher was therefore to gather evidence and to reflect, with school representatives, on what was actually happening, as well as to take new initiatives forward.

Thus whilst particular factors are necessary for the successful implementation of specific strategies, certain preconditions need to be in place before any strategy can succeed. Where the strategies were most successful in raising achievement, these pre-conditions were fully met. Where one or more of the following preconditions was absent, the development of the strategy was inhibited. The preconditions appear to include:

- Leadership support: without the full commitment of the headteacher and senior management team, strategies can only be implemented on a limited basis. Such a commitment means ensuring that sufficient time and resources are allocated so that an area identified for development is followed through. Heads need commitment, not only to the chosen strategy or strategies, but to the whole notion of raising boys' achievement (without, of course, jeopardising the achievement of girls).

- Commitment shared by all staff involved: the project has shown that enthusiasm from the Headteacher is insufficient if staff themselves fail to commit to the chosen approach and to take it fully on board. Thus a sense of teamwork is essential, with values and aims which are transparent, consistent, shared by all adults working in the school and permeating all aspects of school life.

- A supportive but energetic and robust school culture: such a culture would give attention to pupils' and teachers' self esteem and build trust between pupils and adults - creating a 'cycle of affirmation'.

- A clearly articulated ethos: ethos can be a nebulous word, used lightly and often lacking definition. We use it here to mean the creation of an ordered learning environment with clear boundaries, high levels of self-discipline, high but realistic expectations It also embodies an explicit approach to giving reward and praise, where both staff and students feel valued and involved, and where there is a focus on the individual pupil.

- A school culture which emphasises achievement: in such a culture, achievement, interpreted broadly, would be seen as desirable for all students, would be accepted as the norm and would be something to be celebrated. This encompasses a supportive classroom environment which encourages all pupils to be positive about their own and others' achievements, and emphasises pride in work and behaviour, responsibility and independence.

- An emphasis on pedagogic practice, with sustained reflection on teaching and learning, linked to the active use of performance data to inform and monitor practice. 


\section{Factors that impede steady progress}

The previous section has presented an 'ideal type' situation, yet as all schools know, there are many factors which are largely outside their control and that can frustrate the smooth working of the strategies. These include, for example, problems with the recruitment and stability of staff, and the extent to which staff have time to devote to the project in a situation where so much time is spent covering for unfilled vacancies, or on preparing for Ofsted inspections, or implementing various other government initiatives.

Different cohorts of students also change the situation, particularly where sample size is small, as in many primary schools, and schools that can seem totally on track in narrowing the gender gap can see their profile change abruptly with one or two 'poor' years. This issue of comparability between cohorts is crucial, since too much emphasis is sometimes put on year by year variations which may reflect the performances of only a very few students. It is clear, too, that many of the schools we are working with have significant numbers of children with considerable emotional problems, and that despite schools' best efforts, these can continue to affect achievement. The work we have undertaken with schools in challenging circumstances - in inner London, in metropolitan parts of the West Midlands and the North West - suggests that high levels of achievement are possible in such areas. As Mortimore and Whitty (2000) argue, school improvement projects show that committed and talented heads and teachers can improve schools, even if they contain a proportion of disadvantaged pupils.

\section{The importance of a longterm approach}

Above all, the evidence we have amassed during this four-year research project has convinced us that short-term strategies are unlikely to impact positively upon students' achievements in ongoing ways. One of our contentions at the outset of the project was that schools are faced with too many short-term initiatives, so that strategies are often put into place for only a year, or even a term, and if little impact is apparent in the short term, the strategy is abandoned. The Raising Boys' Achievement Project has reinforced our belief in the need for implementation and evaluation over a considerable period of time: only then is it possible to get reliable evidence about what and how a strategy is working, and to understand the factors contributing to sustainability.

The project has used a number of different strategies to assess longer-term impact. These include quantitative measures of change in pupil performance over the course of the project. Using national data-sets, evidence has been gathered on individual pupils' performance in the Key Stage assessments covering KS2 to KS3 and KS3 to GCSE. At the same time, pupils in all the participating schools have completed attitude questionnaires with a view to establishing whether there have been any systematic changes over the duration of the project. Even so, whilst these have proved to be useful in indicating change, the intervention stage of the project took place over a three-year period only, and in most cases it took a year for the strategies to be identified and implemented. In the Partner schools, the various pre-conditions needed to become established and embedded within the fabric of the schools. And, as noted above, progress was in some instances frustrated by factors largely outside the schools' control. It is therefore the case that although we can confidently assert that the strategies we put forward have the 
potential to raise achievement, firm evidence of consistent improvement will only be available after a longer period of time has elapsed.

\section{Comment}

The key outcome of our reflection on the work of the project is the need to look at the bigger picture, and not to see under-achievement within a narrowly defined framework, as something to be 'tackled' through a prescribed set of simple steps, which can be immediately and simply implemented to achieve 'quick fix' solutions.

\section{Across Schools}

\section{Background: competition and collaboration}

'Concerned with the need to raise standards of achievement, and improve their positions in the world economic league tables, governments over the last 20 years have intervened more actively to improve the system of schooling' (Day \& Sachs, 2004, p3)

The government interventions referred to in the quotation above make use of both the stick and the carrot - the hard currency of competition and the softer resonances of collaboration.

Currently the competitive mode is most apparent in the search for 'reputation': one of the pay-offs of a good reputation is the easy recruitment of student numbers - and a major builder of reputation among secondary schools is success in the national league tables of examination results. At the same time we have a celebration of 'networking' and 'community', ways of professional relating that might not be expected to flourish in a climate dominated by competitive practices. Indeed, the collaborative impulse is itself a key dimension of government strategic planning for school improvement and reflects a commitment to structures that enable the sharing of good practice across schools.

The key feature of the Project's research design was the grouping of schools in clusters or 'triads' and the principle that the project worked to was not dissimilar to Michael Apple's idea of 'democratic professionalism' with its 'emphasis on collaborative, cooperative action between teachers and other educational stakeholders' (Apple, 1996; in Day and Sachs, 2004, p7).

The idea of forming collaborative 'triads' as a structure for school improvement came from an earlier project involving secondary schools in one LEA (see Rudduck et al, 2000; Rudduck et al 2003. Here, two or three 'cluster' schools were linked to a 'host' school; the host and 'cluster' schools were all within relatively easy travelling distance of each other; this meant however that they recruited their students from the same catchment area. The fact that they were in competition initially made schools wary of sharing good practice -

giving their ideas to their rivals, as the headteachers saw it (quoted in Rudduck et al, 2000):

Well, there's the competition element. ... I mean, as a teacher, you want to improve all the students in the County but perhaps not the ones in the school next door. ... Every school is striving to be excellent ... but also every school wants its individuality and it doesn't really want all that rubbed off on its neighbours and nor, I guess, do its neighbours. Because how do schools sell themselves? They sell 
themselves as an unusual if not unique blend of things which they feel fit their communities.

In the RBA project the schools in the secondary triads were at some distance from each other while the primary schools, although nearer each other, did not show any signs of recruitment rivalry.

Short interviews were conducted at the final project conferences in July 2003 (secondary teachers) and in September 2003 (primary teachers). The purpose of the interviews was to find out how schools had perceived and experienced the triad structure. All teachers attending the conference were interviewed individually for about 15 minutes - in all, 22 primary school teachers from 8 triads and 18 secondary school teachers from 8 triads $^{2 .} \mathrm{A}$ summary of the interview data (based on a preliminary 'overnight' analysis) was offered at the end of each two day conference so that the teachers who had contributed had some sense of the overall pattern of response.

\section{Focus and findings}

The review of the triad structure was designed to ascertain:

- whether schools understood the rationale for working in triads, whether the Originator role in the triad structure had been sustained and what participants felt about it;

- how participants identified the advantages and disadvantages of working in the triads;

- what kind of support the schools valued.

Overall, the data indicated that

- the idea of working with other schools, over time, on a shared focus, was experienced very positively;

- schools were more comfortable with an equal relationship than with a leader plus follower relationship within the triads;

- in the light of the commitment to equal relationships in the majority of the triads, the rationale for the Originator role became sidelined; while this was difficult in terms of the research design, much was learnt about how schools like to work and the strengths of non-hierarchical relationships.

The responses to these three questions are discussed in turn.

\section{From hierarchy to equality}

As Chapter 3 explains, the initial research design envisaged that the Originator schools, which had demonstrably made some progress in narrowing the gender gap over two to three years, would support their two partner schools in ways of working that had been effective for them. The logic of the plan foundered to some extent on two counts.

Firstly, some Originator schools attributed their success in narrowing the gender gap to a general and all-embracing culture of achievement rather than to specific strategies. It was therefore difficult for them to guide Partner schools except in terms of building a broad culture of achievement in which boys were able to commit themselves to learning. 
However, they claimed that discussions within the project helped them to reflect on possible explanations for their own success.

Secondly, while teachers from the Originator schools were more likely to remember the logic of the triad structure, others either claimed never to have known it (this was particularly true of teachers who joined after the first conference where the rationale was fully discussed) or argued that they put it aside in favour of equity within the group. Again, while two Originator schools felt some disappointment that the role they expected to play - and had felt proud of being chosen by the central team to play - had changed, they found alternative sources of satisfaction (if not of status) and claimed that working with their Partner schools had led them to look more closely at what they had done and to consider what more they needed to do. They also found that their Partner schools could be a source of new ideas. In some triads therefore, the emphasis was, in practice, more on sharing within a group of equals:

(The idea was) to share the practice that was going on in the originator school with the other schools... but in real terms it never actually panned out like that. We're all equivalent.

It seemed actually to be very equal, right from the beginning. ... When we were meeting it was very much a sharing of ideas so it seemed fine; we seemed to work together well.

There was a lead school to begin with but once we were actually in the project it was equal.

In some triads the spirit of equality was strengthened because the teachers from the three schools were all of equal status, whether deputy heads or heads. Equality was also signalled by decisions in the triads to meet, as far as possible, at each school in turn. In short, the triads were virtually all committed to a non-hierarchical relationship: the principle they felt most comfortable with was that of sharing among equals. Only where the lead school was particularly experienced in a pedagogic strategy that had a high national profile (such as preferred learning styles) was the leadership role more readily accepted by the two Partner schools.

This does not of course mean that the work of the Originator schools was not 'transferred' but rather that the traffic in ideas was not just one way.

\section{The benefits of working in a triad}

The benefits that the schools identified went beyond the project's intention to test the transferability of effective practices from one school to others. The great strength of the structure was seen as 'giving ideas, getting ideas'. Teachers thought it 'better than schools going it alone'. They enjoyed the opportunities for argument and discussion and some said that they had not had a chance to 'do anything like this for years'. They felt that they had common ground in 'having the same sense of worry about the way education is going' and they felt together they had the strength to act in ways prompted by their own professional judgement: the triads gave them confidence and support. Additionally, some suggested that working together brought with it a strong mutual accountability and that the project was therefore more likely to be sustained in the current climate of competing pressures and initiatives, so that it 'doesn't let it get overtaken by other things'. This is an important observation. 
Others claimed that working on the project had helped their schools to re-assert the importance of thinking about learning at a time when they were overly preoccupied with bureaucratic business. Again, teachers claimed that the dialogues within the triad meetings and at the conferences enabled them to think and talk 'at a different level to how you usually talk about things'. Teachers welcomed being involved in an activity that was about wider educational rather than narrow performance issues; they enjoyed hearing what other schools were doing and being able to ask questions in cooperative company.

In sum, the benefits were seen as:

- Access to a range of practical ideas.

- Being able to see some of those ideas in action in different settings (visits to other triad schools to observe practice and occasionally to interview students) were much appreciated.

- Opportunities to exchange and evaluate ideas.

- Collegiality and a chance to think about important educational issues together.

- The opportunity to talk about dilemmas and disappointments in sympathetic company.

- Time to reflect on their own practice.

- Sustaining and understanding an initiative over time instead of just over a few weeks or months.

\section{The role of the link researcher}

In teachers' views, the success of the triad structure reflected a mix of three things: the commitment of the teachers involved, the interests and support of the senior management teams and the sustained contact with members of the Cambridge-based research team.

In addition to having support from senior colleagues and feeling comfortable that the initiative was maintaining a high profile in school, teachers particularly valued the support they received from the central team through their link researchers. The ideal of the link researcher that emerged was someone who was multi-skilled. Particularly important, interestingly, was someone to take the organisational lead: setting up meetings, ringing up people and reminding them of deadlines, and taking the administrative burden from teachers who were already busy in schools. Without this being done the triad meetings and the project as a whole - might not have worked so well.

Equally important was a link researcher who set deadlines, and who ensured that they were met, knowing that if there was slippage, because of different priorities in the schools, then the different triads would lose some coherence and the project would suffer. The third skill was the skill of the experienced researcher to interview pupils, analyse and interpret data and comment on different research ideas or possible strategies. Also identified was the important role of keeping the focus of the project constantly in view in a climate where there are multiple initiatives in each school which could easily drown out or mute the commitment to RBA.

\section{Comment}

The appeal of working together on an educational agenda that was widely recognised as of national significance helped to sustain the impetus among the triads, even when schools 
were some distance apart. However, it was apparent that there were different patterns of engagement across the schools and these needed careful handling by the link researcher and by other members of the triad. Individual schools have their own moments of internal concentration and crisis which may not match the rhythm of the triad's or the Project's deadlines or demands.

Not all the triads could be sustained: a few dissolved, or dwindled into diads, and one became a loner but was able to recruit some new partners. There was rather more turbulence among the secondary schools than among the primary schools although in one primary triad the members were 'cohabiting' without being closely engaged with each other. The reasons for the 'dwindling' were usually clear (movement of teachers in the school's RBA team to another school, for example). Nevertheless, even among the diads commitment to the principle of a collaborative structure was still very high, and new participants joining a triad saw the potential for new ideas and energies. However, when a staff member left, the baton was not always passed on smoothly within the school and in some cases there was no explanation of the rationale for the triad structure.

There is still much to learn about setting up and sustaining productive collaborative relationships among schools. It proved important in the both the RBA project and the earlier project (see Rudduck et al 2000, Rudduck et al 2003) to look behind the easy rhetoric of 'networking' to examine such things as status issues within working groups; it was also important to look searchingly at the details of the process - which networking is expected to promote - of transferring ideas and practices from one school to another. 


\section{CHAPTER 12: Concluding Discussion}

This research and intervention project started from a concern about 'under-achieving' boys in primary and secondary schools. As the research team worked over the last four years with mainstream and special schools in different parts of England, so it became obvious that this definition of the 'problem' was far too narrow. The challenges of defining 'underachievement'; the legitimacy (or not) of identifying the characteristics of 'boy' and 'girl' amidst diverse gender constructions; how to identify and value different facets of achievement in school; whether 'gender' was even a legitimate concern to explore within special school contexts; the development (or not) of 'boy-friendly' approaches to pedagogy, curriculum and class organisation; all these questions have confronted us in this research, and the answers have sometimes surprised us.

At the end of the pilot stage of the project (2000-01), we arrived at a fourfold classification of approaches in response to the work which was being developed in schools. This classification (pedagogic, individual, organisational and socio-cultural) continued to inform our thinking through the intervention stage of the project (2001-04), and helped define and structure the various strategies which evolved. These strategies are not independent of each other, however, and there must be integration of approaches if impact is to be maximised. Equally, as we make clear in chapters 8 and 9, we have become convinced that the socio-cultural, combating images of laddish masculinity and establishing a school ethos where these pupils are supported, encouraged and their varied achievements valued, is a crucial underpinning which must be established if the potential of other strategies is to be maximised.

Over the three years of the intervention stage, too, it has become evident that whilst particular strategies have the potential to help raise the achievements of boys and girls, they are not panacea of themselves; without the establishment of necessary pre-conditions, they are likely to be limited in effect.

In the following sections we draw out from the four categories our main conclusions and suggest ways in which the various strategies might be developed in other schools.

\section{Pedagogic approaches}

The main pedagogic approach followed by the RBA project in primary schools focused on literacy. Although there have been recent challenges to the conventional wisdom about boys and literacy, it remains clear from national data and from our case study schools that boys usually do less well than girls in reading and particularly in writing. It is equally clear, however, that any approach to improve boys' motivation, interest and achievement in literacy must explore the complex relationship between product and process, to develop an holistic approach across the whole primary school curriculum.

In one triad, the focus was situated firmly on reading, both to raise boys' self-esteem and achievements, and to emphasise reading as a pleasurable activity. A range of approaches was developed, based on more extensive discussion of texts, teachers modelling ways of responding to the meaning and content of books, and the development of a 'reading buddy' scheme between unmotivated year 6 boys and under-achieving year 3 boys. What emerged was not so much a concern about teaching reading, but a need to focus on encouraging boys to become successful and satisfied readers. This involved not only having a wide 
range of texts available, to stimulate and sustain pupils' interest, but also creating space for talk and reflection about reading, and sharing ideas about the text and what was enjoyable in it. When space and text availability enabled such discussion to take place, standards of reading among boys improved markedly, sometimes by twice that expected within national test parameters. In these schools, opportunities to choose interesting reading matter, and to discuss reading in a meaningful way, were seen to be vital in enabling boys to improve as readers, and in establishing a context in which boys wanted to read.

In two other primary triads, the interventions focused on transforming writing for boys who did not engage willingly or actively with it. The overall strategy emphasised the process of becoming a writer, stressing some of the purposes of writing, such as communicating with a reader and conveying a personal voice. In one triad, an integrated approach to literacy in its broadest sense, seeing reading, writing, speaking and listening as interrelated rather than distinct components, was adopted. Greater attention was paid to paired and group talk, to giving pupils opportunities in different settings to engage in oral preparation for narrative. Pupils were encouraged and taught how to discuss their story lines more explicitly with each other, to explore aspects of character, plot, setting and vocabulary, and to use visual stimuli as a source of inspiration for their writing. The second triad focused directly on drama as a creative context for teaching literacy. Drama was seen as providing opportunities for stronger cross-curricular links, and for offering a greater range of creative and sensory experiences. In particular, it was envisaged that drama would encourage collaboration between pupils, stimulate the imagination and develop empathy. The aim was to create more imaginative contexts for boys' writing, and by 'freeing' boys from their immediate surroundings, to enable them to write in role. In both these triads, analysis of boys' subsequent writing showed marked improvements in the pace and structure of their story writing. Character depiction, the creation of atmosphere within stories and using dialogue as a narrative device, were all aspects of boys' writing which were significantly better as a result of this emphasis on becoming a writer.

These writing-based intervention strategies were extended into a third year, and the strategies diffused into other schools within each LEA. This approach reiterated and validated the approaches developed in these two triads, and stressed the importance of a number of aspects:

- The central importance of talk, giving more attention to speaking and listening as a means of supporting writing. This was particularly evident in the security and confidence which pupils gained by sharing and exploring ideas before writing, so that they could work them out in detail and gain encouragement from others; this fostered an 'I can do' belief amongst children, which is particularly important with the disengaged and unsure pupil.

- The advantages to be gained through companionable writing with response partners and through group work.

- Teachers who were prepared to risk-take to bring more creativity and variety to literacy.

- Teachers who stressed the need to model writing and show how it might be constructed. 
- More integrated use of ICT so that quality presentation could be more easily achieved, and drafts amended with more ease.

Throughout our work on this project, we have argued for a wider interpretation of achievement than a definition based solely on academic results and national curriculum test outcomes. Equally, though, literacy is a key skill which determines the academic success or failure of many children in their subsequent schooling. It is significant, then, that many of these strategies not only stimulated boys' interest and engagement as writers and readers, listeners and speakers, but also impacted positively upon their academic performances in key stage 2 tests. There were remarkable gains in teacher assessed reading scores in the first triad, for example, with well over half the boys achieving at least one National Curriculum level higher at the end of year 6 than at the beginning of the year, and considerable value-added gains for boys in writing in the two other triads.

A second focus of our work on pedagogy was developed around preferred learning styles. In this context, it is important to stress that our research raises as many questions as it answers, and leads us to caution against some of the claims made by advocates of accelerated learning and multiple intelligences. Certainly there is little evidence in the research we have conducted to suggest that the dominant learning styles of boys differ from those of girls. It is also far from straightforward to analyse classroom activities in terms of specific, self-contained teaching styles, since many pedagogic activities engage different modalities, and may be interpreted differently by different students. Students have also suggested to us that their own learning styles have changed and evolved through time, in response to quality teaching which has helped them to learn in different ways.

These cautionary words are important, because too often the learning style movement has been promoted in schools by enthusiasts, returning from conferences inspired and enthused, and subsequent implementation has sometimes been simplistic and misconceived. The view, for example, that more boys are predominantly kinaesthetic learners and more girls predominantly visual or auditory learners is not supported by our evidence, and such a presupposition can close down more learning opportunities than it opens up.

As we stress in chapter 5, however, our research does suggest that there are gains which can be made, and innovations which can help support learning and transform achievements, where such an approach is implemented carefully and holistically. Where learning styles' approaches have been most effective, classroom observations and interviews with pupils / students and with teachers have identified a number of important issues:

- Developing with teachers and students an understanding of how learning takes place, through keynote presentations to staff and students about different modes and styles of learning.

- Fostering an understanding in students that they have different learning styles, that some may be more prominent than others, but that successful learning involves students accessing different learning styles.

- Creating support for teachers so that they are able to plan lessons which encompass different learning styles, and help them to become more creative in their teaching, planning and assessing, 
This last point is a crucial one, perhaps, because it situates the discussion in the context of pedagogy. Work on preferred learning styles is misconceived if it does not translate into teaching style. In this RBA project, where the emphasis on preferred learning styles was making most impact on students' motivation and learning, students were aware of ways in which teachers were trying explicitly to change their teaching styles:

She uses background music, Mozart and stuff, to create atmosphere ... and opera and classical stuff to create a relaxed background feel ... it's calming and good.

We have kinaesthetic activities which involve relays and boardwork and lots of activity ... the movement is good, it frees you up, helps you to think and to see things better.

It was so good because we understood what he was trying to do ... he explained how we was trying to help us learn it ... when he play-acted, it was like we were talking to Macbeth, not to him ... it was so real.

Teaching became a dialogue in such contexts, with teachers sharing with their students not only what their objectives were, but how they hoped to reach them. Such an openness had several important side effects. It often created a sense of partnership with the students, and a sense of goodwill in that students understood that the lesson had been carefully designed and structured for them in the light of their needs. On occasions, it also fostered a collaborative environment especially when teachers suggested that they were trying a new technique, deliberately engaging with a teaching style which was new to them in order to help the students learn better. 'More teachers should definitively do it', suggested one year 11 boy, 'because it helps us learn better ... it's about variety, change, changing tack when we don't get something, realising what we need to help us learn ... that's what good teachers do'.

\section{Individual Approaches}

As we outline in chapter 6, one of the most intriguing issues we have encountered about individual approaches is the extent to which they have differential impact in different schools. In some schools, target-setting and mentoring have been transformative in their effects upon motivation, engagement and achievement; in others, they have had minimal impact. There are also dangers in such approaches which must be acknowledged. There is the temptation to focus scarce resources of teacher time and energy solely on a minority of students, usually boys, who hover around the $5 \mathrm{~A}^{*} \mathrm{C}$ grade benchmark at GCSE, or around level 4 in key stage 2 English; other, equally deserving students, can be ignored and become devalued. Some mentoring schemes eventually become counter-productive, exerting pressure on students which eventually becomes oppressive and demotivating. Equally, others lack conviction for students, with staff or outside mentors who, whatever their initial good intentions, become too busy to fulfil regular commitments, or who adopt a demeaning or 'bullying' approach when mentees are slow to respond to 'obvious' guidance and advice.

Our research with schools therefore reinforces the belief that mentoring and target-setting are delicate and sensitive matters, which need to be built around mutual understanding and shared commitment. How to mentor successfully is not obvious or simply a matter of commonsense. Process and context are vital, and mentors need help in establishing rapport with mentees, and in developing relationships which are different from those which 
normally characterise teacher-student relationships, and which are perceived by students as genuine and caring for them as individuals.

Many schools devote a great deal of resource to organising and developing target-setting and mentoring. In our experience, there are a series of crucial pre-conditions which need to be established if such schemes are to be credible with staff and students, and if their potential is to be maximised. We want to stress this point; some mentor schemes waste scarce resources because they are not founded around these essential foundations, and in such cases, the reactions of some students and some staff suggest that time and energy could be better spent on other initiatives.

Evidence from the two secondary triads suggests that these pre-conditions have two complementary aspects, related on the one hand to effective and realistic target-setting, and on the other to supportive and assertive mentoring. Target-setting is at the heart of the issue because such targets need to be both realistic and challenging. In some schools, the use of historic data on achievement is not enough, because such data might relate to past expectations of pupils which have been too low, or based upon past achievements of students which have under-achievement embedded within them. It takes time and patience, therefore, to reorientate expectations and to establish a profile of achievement within a school which is based upon higher expectations and realisable within a context of rising self-belief and confidence of staff and students. Value-added data, properly contextualised within local and regional settings, can help to challenge assumptions and raise expectations, as we have clearly seen exemplified in one triad. Equally though, the successful secondary school will give responsibility to staff, within subjects departments, to address target-setting on a regular and frequent basis, and will set aside protected time to enable staff to engage in professional dialogue about learning at the level of the individual child, to ensure that the targets set are realistic and achievable.

The tone and context of the mentoring process are equally crucial. In the secondary triads the perspective of students and ultimate academic outcomes suggest that mentoring has been most effective when it has:

- Been practised by mentors who can establish and sustain rapport with students, who can encourage, support, direct and challenge, without becoming condescending and counter-productive.

- Involved senior staff who are able to protect time to establish a regular dialogue with students.

- An in-built element of negotiation between mentor and subject staff, to enable mentors both to mediate on behalf of 'their' student and subsequently, having addressed an issue identified by the student, to challenge 'their' student to achieve more.

- A mix of styles from each mentor - collaborative and supportive on the one hand offering strategies, advice and encouragement, and challenging and demanding on the other.

- Been framed within a context which allows disengaged students to protect their own images and their own construction of a laddish masculinity or a ladette-like femininity. 
A mentoring strategy based on these characteristics is distinctive. It makes assertive and explicit demands on students, but at the same time it enables the real purpose of mentoring to be fulfilled. It allows students to develop as independent learners, so that they can make an effective transition to future learning, and participate as proactive partners in learning.

\section{Whole School Organisational Issues}

We have stressed earlier (chapter 7) the importance of whole school approaches, with schools attempting to develop an ethos and culture where achievement in many different areas is celebrated and accepted as the norm. One of the essential conclusions we have reached through the RBA Project, is that 'under-achieving' boys and girls are not likely to engage with learning if schools simply concentrate on adopting narrowly focused and quick-fix solutions in isolation from the ethos of the whole school. Students are more likely to respond positively when they feel valued and supported within the whole school context, and feel that the school - as an organisation - is working with their best interests at heart.

How the students are grouped for learning is another aspect of organisational approaches considered during the Project. Whilst many schools, primary as well as secondary, are grouping pupils at an earlier and earlier age according to perceived ability, there remains little evidence which suggests that children in classes setted according to ability learn more effectively than children in mixed ability classes. Conversely, there is continuing resistance to the idea of single-sex classes in co-educational schools, despite emerging evidence to suggest that some girls and some boys are more comfortable in such classes and often attain at higher academic levels.

We acknowledge that the debate on single-sex classes is contentious, and that the evidence is mixed. We recognise that in some schools we have worked with, the challenges of teaching some boys in single-sex classes have impacted negatively upon the learning of other boys. Equally, we agree that there are dangers of stereotyping, of establishing a macho gender regime and of discriminating against some boys in such classes.

Nonetheless, the evidence from other contexts is compelling. Freed from concerns about image, appearance and the need to 'perform to role', boys and girls have repeatedly described the advantages to be gained from being taught in single-sex classes: a willingness to engage more in discussion and questioning, being prepared to discuss emotions and explore feelings, a readiness to participate without fear of scorn or discomfort. When such classes have been taught effectively and sensitively, achievement levels have risen markedly, for both boys and girls, particularly when the strategy has been carefully targeted at the needs of particular students studying particular subjects.

The differential effect of this strategy in different schools leads us to identify a series of pre-conditions for implementation. These pre-conditions seem crucially important in any attempt to implement single-sex classes for particular subjects, because our experience from the RBA project suggests that without them the initiative is almost bound to fail. Within the classroom, these pre-conditions suggest that:

- The pedagogy must be interactive, lively and clearly structured, based around high levels of teacher input, and moving the lesson on with pace and clarity. 
- Teachers must use a proactive and assertive approach, which avoids the negative or confrontational, conveys high expectations and a sense of challenge, and uses praise regularly and consistently.

- There must be the promotion of a team ethic, to forge an identity for the class of which the students can feel part, with humour and informality, and identification with students' interests and enthusiasms.

What our research on the RBA project does not suggest, however, either in the context of mixed-sex or single-sex teaching, is that there is a case for boy-friendly pedagogies. We accept that the pedagogy described above is sometimes described as boy-friendly, but it is equally girl-friendly. It defines the characteristics of quality teaching, which is just as suitable and desirable for girls as for boys. Likewise, the emphasis on competition is no more likely to motivate or demotivate particular boys than it is to motivate or demotivate particular girls.

What is important within any school, however, is that senior managers identify proactively with the single-sex initiative, not in terms of an 'experiment' which is being supported and enabled in a permissive style, but of active support and engagement with the initiative. Certainly, in the schools where this strategy was most effective, parents and carers, students themselves, all staff - teaching, administrative and support staff - had been engaged in the debate, and headteachers themselves had vigorously promoted and helped sustain the initiative through time. Proactivity and public support were the watchwords.

There are other aspects of organisational issues which we suggest are important in establishing a whole-school environment which expects, acknowledges and applauds achievements. In several schools, for example, a Leadership (or Prefects) System in year 11 was seen as a key element in affecting both the motivation and self-esteem of students, and subsequent achievement levels. A further characteristic of organisational strategies was the emphasis on a high quality of aesthetics around the school environment, with high priority placed on the visual in terms of wall displays of students' work. Public recognition of success was also high, through Achievement Boards which carried photographs and pen portraits of boys and girls who had achieved success not only in the academic, but also in the sporting, drama, musical and community contexts. The aim is to make all students proud to be publicly acclaimed and recognised for their achievements, and to establish acceptable and non-stereotypical role models for other students. Displays of current quality work in public areas, a formal merit and award system, and 'student of the month' awards, all contributed to sustaining an achievement culture within schools.

\section{Socio-cultural issues}

Most of the schools involved in developing socio-cultural strategies were located in challenging urban catchment areas with high levels of social deprivation, and yet in each triad there have been at least two schools where the strategies were transformative in their impact upon motivation, engagement and academic achievement of 'under-achieving' pupils. Significantly, not all of the strategies themselves were novel or innovative, and the form they took varied from school to school, with one triad developing citizenship initiatives, one focusing on Arts, one implementing a paired reading scheme and another focusing on key leaders. What was unusual and original about them, however, was the rationale underpinning their implementation, and the sense of importance which teachers 
placed on them, so that in most schools in these triads, the socio-cultural approaches which were developed have become central to the life and ethos of the school.

Those schools where socio-cultural strategies were most transformative were those where headteachers recognised that there were sometimes conflicts between the cultural contexts of home and school, and that such conflicts might lead to disengagement and potential under-achievement. Recognition of the problem was then translated into action, the focus of which was to develop an inclusive ethos which sought to engage and motivate all pupils to become fully involved in school, and to develop the kind of self-esteem which was based on successful learning, rather than on values which conflicted with, and inhibited, academic learning. The strategies themselves thus became not only an integral part of each school's ethos, but were also embedded in the curriculum, and central to teaching and learning.

The targetting of initiatives on disengaged and potentially under-achieving boys took different forms. In the secondary triad, the approach was an innovative one, focusing initially on key leaders, on those students whose physical presence, manner and behaviour exerted power and influence within the peer group. Recognising the significance of these key leaders within the peer group, schools sought to define how many of these students could be won over and positively involved in the life of the school. The identification of key befrienders, informal mentors with a more wide-ranging brief to acknowledge, integrate and offer subtle support to these students, was one socio-cultural strategy which was successful in helping to effect change in some secondary schools.

Attempts to transfer this strategy to other schools in similar socio-economic contexts proved immensely challenging, however. At one level, it is crucial that the characteristics of and interactions within the student year group are well-known to year heads, so that appropriate judgements can be made about who are the key student opinion formers in any year. Balanced against this has to be the potential of the key leaders to respond positively to initiatives the school puts in place, since, as we make clear in chapter 8 , there are occasional situations when key leaders are so utterly anti-school in their attitudes and behaviours that they are unlikely to be responsive. The choice of key befriender is equally crucial, with the emphasis upon credibility, rapport, identification with student potential, persuasion and non-stereotyping attitudes and behaviour. Important too, is the balance between prescription and supportive proactivity.

Central to the success of this strategy in the Originator school was the creation of a school 'house' style, with emphasis on uniform, on regular attendance and responsive behaviour monitoring, and on the school day as a time of learning rather than social activity - all of which can help to create a positive ethos of high expectations. At the same time, School Councils which respect students' voices and respond positively to issues they identify, contribute to an environment where students feel valued and included.

Giving pupils space to articulate their feelings and emotions, seeking to find something for each one to excel in, and getting all pupils fully integrated into school life were also priorities underpinning the socio-cultural strategies in the primary triads. As with the key leader strategy, such initiatives take time to become embedded and to become part of the underlying ethos of the school. They involve a willingness on the part of Headteachers to acknowledge under-achievement and to use familiar activities creatively and imaginatively to target it. At times this means taking risks to engage individual pupils in roles where 
they are actively supported to make choices and to achieve success. This can only happen where all staff are fully committed to putting agreed strategies into practice. When these preconditions are in place, research in the primary triads showed that it is possible to generate a sense of inclusiveness for under-achieving pupils, at the same time improving communication skills, developing an increased sense of responsibility, and contributing to an increasing sense of confidence and positive self-image and to more highly developed social skills. Furthermore, however much we might agree that key stage 2 test outcomes offer only one narrow perspective on achievement, it is the case, as we show in chapter 9, that these socio-cultural initiatives, where they have been applied consistently across the school have impacted positively upon academic outcomes at key stage 2 and led to enhanced academic achievement.

\section{Interventions within Special Schools}

The long-established emphasis on an individualised approach within special education, regardless of gender, and yet at the same time, the numerical predominance of boys within most special schools, meant that it was difficult initially to identify the contribution which the Project might make within the context of special schools. Once defined, however, it became clear that many of the intervention strategies had close parallels with those developed within the mainstream project. Thus the holistic approach to improving boys' writing, through visual and aural approaches, and scaffolding of tasks, replicated some of the developments in those triads exploring primary literacy issues (chapter 4). Similarly, the development of pedagogy which took account of work on learning styles and active learning, mirrored some of the developments in three mainstream (two secondary and one primary) triads (chapter 5). Equally, the emphasis on evolving whole school approaches which responded effectively to pupils with low self-esteem, and a developing disaffection because of their very placement in special schools, was analogous to the socio-cultural interventions developed particularly in the primary triads (chapter 8 and 9).

As we have explored in the previous chapter, however, there are two significant differences which emerge when we consider the intervention strategies developed within the special school contexts. One is the overriding concern with the individual which, although having some equivalence with the individual strategies developed in some secondary triads (chapter 6), is much more high profile, persistent and overriding within the strategies developed in the special schools. In the special schools, the social and personal well-being of the individual, as well as the academic achievement of individual pupils, is the pivotal consideration.

A second difference relates to the integration of strategies. We acknowledge in chapter 3 that our fourfold classification of intervention strategies is to some degree an artificial construct, with inevitable overlap between the categories in different contexts. This is nowhere better illustrated than in the special schools, where individual and organisational considerations underpin all initiatives, and where it is frequently impossible to separate pedagogic considerations from individual ones, or socio-cultural issues from organisational initiatives. In the context of special schools, the need for integration of different approaches, within a holistic whole school framework which focuses on the needs and well-being of the individual, is the central message from the Project. 
It is worth reiterating the conclusions reached in chapter 10, however, and confirming that emerging research, both within the context of this Project and elsewhere, does not lend support to the notion that there is a case for a separate special education pedagogy; 'the priority is to use special education knowledge to develop inclusive pedagogy' ( $p$ 129, this report).

\section{The broader issues}

Whilst preceding discussion has focused on specific strategies, broader findings also emerged from the project.

Firstly, the triad structure, where schools worked on a shared focus in a generally nonhierarchical relationship, was experienced very positively. Schools enjoyed being able to access a range of practical ideas and to see some of these in action in different settings. They valued the chance to think about important educational issues and to reflect on their own practice, and to sustain and develop understanding of an initiative over a period of time.

Secondly, the project highlighted the importance of school and community contexts, where there are different routes to achievement, and alternative ways forward. Successful strategies emerged that schools found appropriate to their own particular contexts and implemented through collaboration, rather than through imposition. They were willing to explore in depth aspects of 'under-achievement', to understand the complexity of the issue and its varied gender dimensions, and to take risks in developing innovative approaches specific to each local context.

Thirdly, while there is a set of pre-conditions necessary for the successful implementation of each of the strategies discussed above, other preconditions are necessary before any strategy can succeed. These include leadership support and commitment by the staff involved, a whole school atmosphere and clearly articulated ethos where there are high expectations and where all feel valued and involved, a culture which celebrates achievement in its widest sense, and an emphasis on pedagogic practice.

\section{Conclusion}

The RBA research has shown that there is no 'magic dust', as one Headteacher requested, to 'solve' the gender gap in achievement in schools. We are nevertheless confident that the intervention strategies, put in place by participating schools in contrasting socioeconomic environments across England, can be effective in raising boys' achievement. What such successful strategies also do, however, is to raise girls' achievement too, and so in many instances the gap - at least in the short term - is potentially perpetuated. We would not have it otherwise, for a fundamental parameter within which we have worked is that the strategies endorsed by the Project should not in any way be detrimental to girls in either an academic or a social sense.

Furthermore, whilst we assert that the strategies we have chosen to monitor have been effective in certain contexts, they cannot, as we have been at pains to point out, be implemented on a short-term basis. They take time to develop and to become embedded if they are to be sustained and work effectively. Neither can they be implemented without regard to the necessary preconditions we have explored in the preceding chapters. The 
choice of an appropriate strategy must relate to the specific school context: a primary school where there is under-achievement in literacy may decide to focus its efforts there, while a school with a significant proportion of disaffected students may choose a sociocultural strategy. Nevertheless, while a school may choose a specific strategy, it is important to bear in mind that all depend at the most basic level on inspiring, imaginative and exciting pedagogy which generates enthusiasm for learning and achievement, and on a school ethos which encourages and facilitates achievement in its widest sense. 


\section{REFERENCES}

Archer, J. \& Lloyd, B. (2002) Sex and gender, Cambridge: Cambridge University Press

Arnot, M., David, M. \& Weiner, G. (1999) Closing the Gender Gap: Postwar Education and Social Change, Cambridge: Polity Press

Arnot, M., Gray, J., James, M. \& Rudduck, J. (1998) Recent Research on Gender and Educational Performance, London: Office for Standards in Education

Askew, S. \& Ross, C. (1988) Boys don't cry: Boys and sexism in education, Milton Keynes: Open University Press

Barker, B. (1997) Girls' world or anxious times: what's really happening at school in the gender war? Educational Review, 49, pp. 221-228

Baron-Cohen, S. (2003) Men, women and the extreme male brain, London: Penguin

Barrs, M. (1998) Texts and Subtexts in Boys and Reading, London: Centre for Language in Primary Education

Barrs, M. \& Cork, V. (2001) The Reader in the Writer, London: Centre for Language in Primary Education

Barrs, M. \& Pidgeon, S. (eds) (1993) Reading the Difference: Gender and Reading in the Primary School, London: Centre for Language in Primary Education/London Borough of Southwark

Barrs, M. \& Pidgeon, S., (eds) (2002) Boys and Writing, London: Centre for Literacy in Primary Education

Barton, A. (2000) Raising boys' achievement in modern foreign languages through single-sex grouping. Paper presented at the British Educational Research Association Annual Conference, University of Cardiff, September 2000

Bearne, E. (2002) Multimodal narratives, in M. Barrs \& S. Pidgeon (eds) Boys and Writing, London: Centre for Literacy in Primary Education

Bearne, E., Dombey, H. \& Grainger, T., (2003) Classroom Interactions in Literacy, Maidenhead: Open University Press

Bell, D. (2004) The Achievement of Girls, Speech to the Fawcett Society, 8 March

Benjamin, S. (2003) Gender, 'special educational needs' and inclusion, in M. Nind, K. Sheehy $\&$ K. Simmons (eds) Inclusive Education: Learners and Learning Contexts, London: Fulton, pp. $247-260$

Biddulph, S. (1998) Raising Boys: Why boys are different - and how to help them become happy and well-balanced men, Sydney: Finch

Blunkett, D. (2000) quoted in 'Single sex classes to help failing boys', The Observer, 20 August, p. 4

Boaler, J. (1997) Reclaiming school mathematics: the girls fight back, Gender and Education 9, pp. $285-305$ 
Cline, T. \& Ertubey, C. (1997) The impact of gender on primary teachers' evaluations of children's difficulties in school, British Journal of Educational Psychology, 67, pp. 447-456

Coffield, F., Moseley, D., Hall, E. \& Ecclestone, K. (2004) Should we be using learning styles? What research has to say to practice, London: Learning and Skills Research Centre

Colley, H. (2003) Engagement mentoring for 'disaffected' youth: a new model of mentoring for social inclusion, British Educational Research Journal, 29, pp. 521-542

Connell, R. (1989) Cool guys, swots and wimps: the interplay of masculinity and education, Oxford Review of Education 15, pp. 291-303

Connellan, J., Baron-Cohen, S., Wheelwright, S., Batki, A. \& Ahluwalia, J. (2000) Sex differences in human neonatal social perception, Infant Behaviour and development, 23, pp. $113-118$

Croll, P. \& Moses, D. (2000) Special Needs in the Primary School: One in Five? London: Cassell

Daniels, H., Hey, V., Leonard, D. \& Smith, M. (2000) Issues of equity in special needs education as seen from the perspective of gender, in H. Daniels (ed) Special Education Reformed: Beyond Rhetoric? London: Falmer Press, Chapter 3

Davis, P. \& Florian, L. (2004) Teaching Strategies and Approaches for Pupils with Special Educational Needs: A Scoping Study, Research Report 516, London: DfES

Day, C. and Sachs, J. (2004) Professionalism, performativity and empowerment: discourses in the politics, policies and purposes of continuing professional development, in C. Day \& J. Sachs (eds) International Handbook on the Continuing Professional Development of Teachers, Open University Press, pp. 3-10

Deem, R. (1980) Schooling for Women's Work, London: Routledge \& Kegan Paul

DfES (2001) Special Educational Needs Code of Practice, London: DfES

DfES (2003) Excellence and Enjoyment: A Strategy for Primary Schools, London: DfES0377/2003

DfES (2004a) Key Stage 2 to GCSE / GNVQ: The Value Added Pilot, London: DfES.

DfES (2004b) The Autumn Package, 2004: Pupil Performance Information. London: DfES

DfES (2004c) www.standards.dfes.gov.uk/thinkingskills/resources, last accessed 9 August, 2004

DfES / The London Challenge (2004d) Key Stage 3 National Strategy: Ensuring the Attainment of Black Caribbean Boys, London: DfES

DfES (2004e) Statistical First Release (SFR 44/2004)

Downing, D., Johnson, F. \& Kaur, S. (2003) Saving a Place for the Arts? A Survey of the Arts in Primary Schools in England, London: NFER, Local Government Association Research Series 41

Epstein, D., Elwood. J, Hey, V. \& Maw, J. (1998) Failing Boys? Issues in Gender and Achievement, Buckingham: Open University Press 
Evans, J., Harden, A. \& Thomas, J. (2004) What are effective strategies to support pupils with emotional and behavioural difficulties (EBD) in mainstream primary schools? Findings from a systematic review of research, Journal of Research in Special Educational Needs, 4, pp. 216

Fennema, E. (1996) Scholarship, Gender and Mathematics, in P. Murphy \& C. Gipps (eds) Equity in the Classroom: Towards Effective Pedagogy for Girls and Boys, London: Falmer Press, pp. 73-80

Fergusson, D. \& Horwood, L. (2003) Resilience to childhood adversity: results of a 21-year study, in S. Luthar (ed) Resilience and Vulnerability: Adaptation in the context of childhood adversities, Cambridge: Cambridge University Press, Chapter 6

Florian, L., Rouse, M., Black-Hawkins, K. \& Jull, S. (2004) What can national data sets tell us about inclusion and pupil achievement? British Journal of Special Education, 31, pp. 115-121

Francis, B (2000) Boys, Girls and Achievement: Addressing the classroom issues, London: Routledge / Falmer

Francis, B. \& Skelton, C. (2001) Investigating Gender: Contemporary Perspectives in Education, Buckingham: Open University Press

Frater, G. (2000) Securing Boys' Literacy: a survey of effective practice in primary schools, London: Basic Skills Agency

Freides, D. (2001) Developmental Disorders: a neuropsychological approach, Oxford: Blackwell

Frosh, S., Phoenix, A. \& Pattman, R. (2002) Young Masculinities, Basingstoke: Palgrave

Gardner, H. (1983) Frames of Mind, New York: Basic Books

Gardner, H. (1999) Intelligence Reframed: Multiple Intelligences for the $21^{\text {st }}$ Century, New York: Basic Books

Gilbert, P. \& Gilbert, R. (2001) Masculinity, inequality and post-school opportunities: disrupting oppositional politics about boys' education, International Journal of Inclusive Education, 5, pp. 1-13

Gillborn, D. \& Mirza, H. (2000) Educational Inequality: mapping class, race and gender, a synthesis of research evidence, London: Office for Standards in Education

Gillborn, D. \& Youdell, D. (2000) Rationing Education: policy, practice, reform and equity, Buckingham: Open University Press

Gipps, C. (1996) Review and conclusions: a pedagogy or a range of pedagogic strategies? in P. Murphy \& C. Gipps (eds) Equity in the Classroom: towards effective pedagogy for girls and boys, London: Falmer Press, pp. 260-271

Gold, K. (1995) Hard times for Britain's lost boys, New Scientist, 4 February

Gorard, S., Rees, G. \& Salisbury, J. (2001) Investigating the pattern of differential achievement of boys and girls at school, British Educational Research Journal, 27, pp. 125 139

Gorard, S., Rees, G. \& Salisbury, J. (1999) Reappraising the apparent underachievement of boys at school, Gender and Education, 11, pp. 441 - 454 
Goss, P. (2003) The gender mix among staff in schools for pupils with severe and profound and multiple learning difficulties and its impact, British Journal of Special Education, 30, pp. $87-92$

Griffin, C. (1985) Typical girls? Young Women from school to the Job Market, London: Routledge \& Kegan Paul

Gurian, M. (2001) Boys and girls learn differently! San Francisco: Jossey-Bass

Harris, S., Wallace, G. \& Rudduck, J. (1993) "It's not just that I haven't learnt much. It's just that I don't understand what I'm doing": metacognition and secondary school students, Research Papers in Education: Policy and Practice, 10, pp. 254 - 271

Hay McBer (2000) Research into Teacher Effectiveness, London: DfEE

Hey, V., Creese, A., Daniels, H., Fielding, S. \& Leonard, D. (2000) Questions of collaboration and competition in English primary schools: pedagogic sites for constructing learning, masculinities and femininities. Paper presented to British Association Educational Research Conference, Cardiff, September.

Hill, J. (1994) The Paradox of Gender: sex stereotyping within statementing procedure, British Educational Research Journal, 20, pp. 345-355

Hirom, K. \& Mitchell, G. (1999) The Effect of Mentoring on the Academic Achievement of Boys. Paper presented to BERA Annual Conference, Sussex, September

Hopkins, D. (2001) School Improvement for Real, London: Routledge Falmer

Jackson, C. (1999) Underachieving boys? Some points for consideration, Curriculum, 20, pp. 80-85

Jackson, C. ( 2002) 'Laddishness' as a Self-Worth Protection Strategy, Gender and Education, 14, pp. 37-51

Jackson, C. (2003) Motives for 'Laddishness' at School: fear of failure and fear of the 'feminine', British Educational Research Journal, 29, pp. 583-598

Jackson, C. (2004) 'Wild' girls? An exploration of 'ladette' cultures in secondary schools. Paper presented at the British Educational Research Association Conference, Manchester, September.

Jackson, C. \& Smith, I. (2000) Poles apart? An exploration of single-sex and mixed-sex educational environments in Australia and England, Educational Studies, 26, pp. 409-421

Jordan, E. (1995) Fighting Boys and Fantasy Play: the construction of masculinity in the early years of school, Gender and Education 7, pp. 69-85

Kenway, J. \& Willis, S. with Blackmore, J. \& Rennie, L. (1998) Answering Back: girls, boys and feminism in school, London: Routledge

Kenway, J. (1995) Masculinities in Schools: Under Siege, On the Defensive and Under Reconstruction? Discourse: Studies in the Cultural Politics of Education, 16, pp. 59-79

Kenway, J. (1997) Taking Stock of Gender Reform Policies for Australian Schools: past, present and future, British Educational Research Journal, 23, pp. 329-344 
Lee-Potter, E. (2003) Rescue plan for the weaker sex, Secondary Education, April 3, pp. 1011.

Lucey, H., Brown, M., Denvir, H., Askew, M. \& Rhodes, V. (2003) Girls and boys in the primary maths classroom, in C. Skelton \& B. Francis (eds) Boys and Girls in the Primary Classroom, Maidenhead: Open University Press

Mac an Ghaill, M. (1994) The Making of Men: masculinities, sexualities and schooling, Buckingham: Open University Press

MacBeath, J. \& Moos, L. (eds) (2004) Democratic Learning: the challenge to school effectiveness, London: Routledge Falmer

Mahony, P. (1985) Schools for the Boys? Co-education Reassessed, London: Hutchinson

Malcolm, L. \& Haddock, L.(1992) "Make trouble: get results": provision for girls in support services, Educational Psychology in Practice, 8, pp. 97-100

Marsh, J., \& Millard, E. (2000) Literacy and Popular Culture, London: Paul Chapman Publishers

Martino, W. \& Berrill, D. (2003) Boys, Schooling \& Masculinities: interrogating the 'Right' ways to educate boys, Educational Review, 55, pp. 99-117

Martino, W. \& Meyenn, B. (2002) 'War, Guns and Cool, Tough Things': interrogating singlesex classes as a strategy for engaging boys in English, Cambridge Journal of Education, 32, pp. 302-324

Martino, W. \& Pallotta-Chiarolli, M. (2003) So What's a Boy? Addressing Issues of Masculinity and Schooling, Buckingham: Open University Press

McDonagh, P. (2000) Diminished men and dangerous women: representations of gender and learning disability in early- and mid-nineteenth-century Britain, British Journal of Learning Disabilities, 28, pp. 49-53

McDowell, L. (1992) Doing Gender: Feminism, Feminists and Research Methods in Human Geography, Transactions of the Institute of British Geographers, NS17, pp. 399-416

Millard, E. (1997) Differently Literate: Boys, Girls and the Schooling of Literacy, London: Falmer Press

Morrison, I., Everton, T. \& Rudduck, J. (2000) Pupils helping other pupils with their learning: cross-age tutoring in a primary and secondary school, Mentoring \& Tutoring, 8, pp. 187-200

Mortimore, P. \& Whitty, G. (2000) Can School Improvement Overcome the Effects of Disadvantage? in T. Cox (ed) Combating Educational Disadvantage, London: Falmer Press, pp. 156-176

Moss, G. (2000) Raising Boys' Attainment in Literacy: some principles for intervention, Reading Vol. 34 no.3 pp101-106

Moss, P. (2002) Feminist Geography in Practice: Research and Methods, Oxford: Blackwell

Myers, K. (2000) Whatever Happened to Equal Opportunities in Schools? Gender Equality Initiatives in Education, Buckingham: Open University Press 
Myhill, D. (2000) Gender and English: are we wearing the right glasses? The Secondary English Magazine Vol. 4 No. 5 December, pp. 25-27

Office for Standards in Education (1993) Boys and English, London: HMSO

Office for Standards in Education (2002) The curriculum in successful primary schools, London: HMSO

Office for Standards in Education (2003a) Yes he can: Schools where boys write well, Ofsted Publications Centre

Office for Standards in Education (2003b) Expecting the Unexpected: Developing creativity in primary and secondary schools, Ofsted: e-publication

Ohrn, E (2001) Marginalization of democratic values: a gendered practice of schooling? International Journal of Inclusive Education, 5, pp. 319-328

PNS/UKLA (2004) Raising Boys' Achievements in Writing. London: Primary National Strategy and United Kingdom Literacy Association

Pickering, J. (1997) Raising Boys’'Achievement, Stafford: Network Educational

Pyatt, G. (2002) Cross-school mentoring: Training and implementing a peer mentoring strategy, Mentoring \& Tutoring, 10, pp. 171-177

Reay, D. (2001) 'Spice girls', 'nice girls', 'girlies' and tomboys: gender discourses, girls' cultures and femininities in the primary classroom, Gender and Education, 13, pp. 153-166

Reid, K. (2002) Mentoring with disaffected pupils, Mentoring and Tutoring, 10, pp. 153-170

Roberts, H. (1981) Doing Feminist Research, London: Routledge and Kegan Paul

Riddell, S., Baron, S. \& Wilson, A. (2001) Gender and the post-school experiences of women and men with learning difficulties, in B. Francis \& C. Skelton (eds) Investigating Gender: Contemporary perspectives in education, Buckingham: Open University Press, Chapter 7

Rudduck, J. (1994) Developing a Gender Policy in Secondary Schools, Buckingham: Open University Press

Rudduck, J. \& Flutter, J. (2004) How to Improve your School, London: Continuum

Rudduck, J., Berry, M., Brown, N. \& Hendy, L., (2003) Learning about improvement by talking about improvement, Improving Schools, 6, pp. 246-257.

Rudduck, J., Berry, M., Frost, D. with Brown, N. (2000) Learning from other schools in a climate of competition, Research Papers in Education, 15, pp. 259-274.

Rudduck, J., Chaplain, R. \& Wallace, G. (1996) School improvement: what can students tell us? London: David Fulton

Safford, K., O'Sullivan, O. \& Barrs, M. (2004) Boys on the Margin: promoting boys' literacy and learning at Key Stage 2, London: Centre for Literacy in Primary Education

Sewell, T. (1998) Black masculinities and schooling: How Black boys survive modern schooling, Stoke-on-Trent: Trentham Books

Sharpe, S. (1976) Just Like a Girl: how girls learn to be women, London: Penguin

Sims, D. (2002) Mentoring Young People: Benefits and Considerations, Topic, 27, pp. 1-5 
Skårbrevik, K. J. (2002) Gender differences among students found eligible for special education, European Journal of Special Needs Education, 17, pp. 97-107

Skelton, C. (1996) Learning to be 'Tough': the fostering of maleness in one primary school, Gender and Education, 8, pp. 185-197

Skelton, C. (2001) Schooling the Boys: Masculinities and primary education, Buckingham: Open University Press

Skelton, C. (2003) Typical boys? Theorising masculinity in educational settings, in B. Francis \& C. Skelton (eds) Investigating Gender: Contemporary Perspectives in Education, Buckingham: Open University Press

Skelton, C. \& Francis, B (2003) Boys and Girls in the Primary Classroom, Buckingham: Open University Press

Smith, A (2001a) What the most recent brain research tells us about learning, in F. Banks \& A. Shelton Mayes (eds) Early Professional Development for Teachers, London: David Fulton, pp. 106-132

Smith, A. (2001b) The strategies to accelerate learning in the classroom, in F. Banks \& A. Shelton Mayes (eds) Early Professional Development for Teachers, London: David Fulton, pp. 159-177

Smith, A. (1997) Accelerated Learning in the Classroom, Stafford: Network Educational Press

Solsken, J. (1995) Literacy, Gender and Work in Families and in School, Norwood, New Jersey: Ablex Publishing Corporation

Sommers, C. (2000) The war against boys: How misguided feminism is harming our young men, New York: Simon \& Schuster

Stanworth, M. (1987) Girls on the margins: a study of gender divisions in the classroom, in G. Weiner \& M.Arnot (eds) Gender under Scrutiny, London: Hutchinson.

Sukhnandan, L., Lee, B. \& Kelleher, S. (2000) An Investigation into Gender Differences and Achievement: phase 2: school and classroom strategies, Slough: NFER

Swain, J. (2000) 'The Money's Good, The Fame's Good, The Girls are Good'; the role of playground football in the construction of young boys' masculinity in a junior school', British Journal of Sociology of Education, 21, pp. 95-110

Swain, J. (2003) How Young Schoolboys Become Somebody: the role of the body in the construction of masculinity, British Journal of Sociology of Education, 24, pp. 299-314

Swain, J. (2004) The resources and strategies that 10-11-year-old boys use to construct masculinities in the school setting, British Educational Research Journal 20, pp. 167-185

Swan, B. (1998) Teaching boys and girls in separate classes at Shenfield High School, Brentwood, in K. Bleach (ed) Raising Boys'Achievement in Schools, Stoke-on-Trent: Trentham Books

Thrupp, M. (1999) Schools Making a Difference, Buckingham: Open University Press

Tinklin, T., Croxford, L, Ducklin, A. \& Frame, B. (2001) Gender and Pupil's Performance in Scotland's Schools, Edinburgh: University of Edinburgh 
Van Houtte, M. (2004) Why boys achieve less at school than girls: the difference between boys' and girls' academic culture, Educational Studies, 30, pp. 159 -173

Vardill, R. \& Calvert, S. (2000) Gender Imbalance in Referrals to an Educational Psychology Service, Educational Psychology in Practice, 16, pp. 213-223

Warrington, M. \& Younger, M. (1999) Perspectives on the gender gap in English secondary schools, Research Papers in Education, 14, pp. 51-77

Warrington, M. \& Younger, M. (2000) The Other Side of the Gender Gap, Gender and Education, 12, pp. 493 - 508

Warrington, M. \& Younger, M. (2001) Single-sex classes and Equal Opportunities for Girls and Boys: perspectives through time from a mixed comprehensive school in England, Oxford Review of Education, 27, pp. 339-356

Warrington, M. \& Younger, M. (2003) 'We decided to give it a twirl': Single-Sex Teaching in English Comprehensive Schools, Gender and Education, 15, pp.339-350

Warrington, M., Younger, M \& McLellan, R. (2003) 'Under-achieving boys in English primary schools? Curriculum Journal, 14, pp. 184 - 206

West, P. (1996) Boys, sport and schooling: an Australian perspective. Paper presented at University of Cambridge Faculty of Education, November 7

Williams, E. (1995) Lapped by Girls, Times Educational Supplement, 14 July

Wise, D. \& Lovatt, M. (2001) Creating an Accelerated Learning School, Stafford: Network Educational Press

Woodhead, C. (1996) Boys who learn to be losers, The Times, 6 March.

Woods, P (1990) The Happiest Days? How Pupils Cope with School, Basingstoke: Falmer Press

Wray, D., Medwell, J., Poulson, L., \& Fox, R., (2002) Teaching Literacy Effectively in the Primary School, London: Routledge

Younger, M. \& Warrington, M. (1996) Differential Achievement of girls and boys at GCSE: some observations from the perspective of one school, British Journal of Sociology of Education, 17, pp. 299-313

Younger, M. \& Warrington, M. (2002) Single-sex teaching in a Co-Educational Comprehensive School in England: an evaluation based upon students' performance and classroom interactions, British Educational Research Journal, 28, pp. 353 - 374

Younger, M., Warrington, M. \& McLellan, R. (2002) The 'Problem' of 'Under-achieving Boys': some responses from English secondary schools, School Leadership and Management, 22, pp. 389-405

Younger, M, Warrington, M. \& Williams, J. (1999) The gender gap and classroom interactions: reality and rhetoric? British Journal of Sociology of Education, 20, pp. 327-343 


\section{APPENDIX}

\section{Examples of research instruments}

\section{Secondary Project - Interview Schedule for use with pupils in schools undertaking work on Preferred Learning Styles / Multiple Intelligences [see Chapter 5]}

\section{General questions which follow-up issues raised by students in their Year 10 interviews.}

- When we came to the school to interview Y11 students in November, you (or some students) gave us a lot of information about the sorts of lessons you thought you learnt most in and the sorts of lessons you learnt less well in. Have any lessons changed very much since then? Or have any teachers changed their style and way of teaching? If so, how?

- Several of you said that Maths and Science were lessons in which you learnt a lot because of the style of the teacher. Is that still so? Can you summarise what the teachers do in those lessons that make them better for learning?

- So what is the most important factor in helping you to learn well?

- Now you're almost at the end of your GCSE course, how do you feel at the moment about school and learning?

- Do you think girls work and learn differently to boys? If so, how?

\section{The Strategy Itself}

- You've been involved in the work which the school has been developing on preferred learning styles. What can you remember about it?

- Can you remember what your preferred learning styles are? Do you know if this is similar (if so how?) to the PLS of other (girls or boys)?

- What do you think were the best things about it? How has it helped your learning, if at all?

- Now that you know more about different learning styles, what is the best way for you to learn something new?

- For the teachers that help you most, what ways do they use to help you to learn?

- What sorts of things might teachers do more of to help you learn better?

- Was it obvious when the teacher was teaching to suit students who had a particular learning style? If so, how? If so, how did this help your learning?

- What hasn't been good about this strategy (of learning styles) / what did not work so well?

- Are there any ways in which it could have been improved?

- Do you think that other pupils (boys and girls) agree with you about preferred learning styles?

- Is there anything else the school could do which would really help you to learn better?

- Is there something you might be able to do to help yourself do better at school? 


\section{Secondary Project: generic interview guide for use with focus groups of pupils at end of intervention stage}

The following questions, put to 3 friendship groups of boys (a minimum of 3 / a maximum of 5 boys in each group) affected by the strategy, form the core questions for each researcher.

- You've been involved in (name of strategy). What do you think are the 2 best things about it?

- What hasn't been good about it / what's not worked?

- Do you think that other pupils (boys and girls) agree with you?

- What has been the impact of the strategy on you as a learner? How has it affected your learning?

- Are there any ways it could be improved?

- Is there anything else the school could do which would really help you to learn better?

- Is there something you might be able to do to help yourself do better at school?

These generic questions will be supplemented by questions relevant to the particular strategy being undertaken.

\section{Primary Project: interview schedule for use with focus groups of pupils at end of reading buddy scheme [see Chapter 4, Case Study 1]}

\section{The reading scheme}

- How do you feel about the reading scheme, have you enjoyed being part of it?

- What has been the best thing about the reading scheme?

- Has there been a worst thing about the reading scheme? If so, what was it?

- Did you have any worries or any problems with the reading scheme? [If yes explore these]

- Do you think that your buddy has got on well with his reading?

\section{Attitude towards and competence in reading}

- Do you like reading? Why/Why not?

- Do you think you're a good reader? How do you know?

- What makes it difficult to read? What makes it easy?

- Do you prefer to read by yourself or aloud to someone? Why is that?

- What sorts of things do you like to read (at home/at school)? What's a recent favourite?

- Why do you think people need to be able to read? 


\section{Primary Project: generic interview guide for use with focus groups of pupils at the beginning of the intervention stage}

The following questions are intended to provide a guide, so that members of the Research Team are following the same broad format, but there is scope to reframe the questions and to ask supplementary ones, as appropriate.

\section{Part 1 explores pupils' views on gender}

1. Do girls do better than boys in this school? Or do boys do better than girls? Or both the same? [If one sex or the other, ask why.]

2. Are there some things boys are better at than girls; some things girls are better at?

3. Do girls work harder than boys, or the other way round, or the same? [If one sex or the other, explore further.]

4. Do the teachers treat boys and girls the same?

\section{Part 2 explores pupils' views on school and their own achievement}

5. Do you like school?

6. What do you like doing best at school? [Ask about subjects if they don't talk about them, look for non-stereotypical responses and question them.] Is there anything you don't like? Why?

7. Do you think it's important to do well at school? Why (not)?

8. Do you feel think you will do well at school?

9. Do you think you work as hard as you can at the moment?

10. Is there anything that stops you from learning?

11. Is there something you could do yourself to help you to do your work better?

\section{Part 3 focuses on school support}

12. Is there something the school could do to help you improve?

13. Do you think the teachers care about how well you do?

14. Do you think you get lots of praise and encouragement?

15. Do you think the teachers do anything particularly to help boys?

16. What happens if you don't do your work properly? 


\section{Primary Project: questionnaire to Year 5 and Year 6 pupils following shared reading programme [see Chapter 9, Case Study 3]}

\section{What I Thinlk About Shared Reading}

You Are: Boy / Girl $\quad$ Your shared reading partner is: Boy / Girl

\section{To answer tick Yes or No, or write a comment}

\begin{tabular}{|c|c|c|c|}
\hline & & Yes & No \\
\hline (1) & Did you enjoy doing shared reading? & & \\
\hline (2) & Did you get on with your partner? & & \\
\hline (3) & Have you helped your partner? & & \\
\hline (4) & Do you think your partner enjoyed reading to you? & & \\
\hline (5) & $\begin{array}{l}\text { Has your partner got better at reading? } \\
\text { How do you know? }\end{array}$ & & \\
\hline (6) & Do you like reading? & & \\
\hline (7) & Are you a good reader? & & \\
\hline (8) & Has shared reading helped your reading? & & \\
\hline (9) & $\begin{array}{l}\text { If you could, would you like to do shared reading with another } \\
\text { child? }\end{array}$ & & \\
\hline$(10)$ & Can you help other children to learn? & & \\
\hline (11) & Is it important to learning to be a good reader? & & \\
\hline (12) & Do you feel more confident about learning? & & \\
\hline (13) & $\begin{array}{l}\text { Have you learnt new skills by being a shared reader? } \\
\text { What are they? }\end{array}$ & & \\
\hline (14) & $\begin{array}{l}\text { Have you any ideas to improve the shared reading scheme? } \\
\text { If so what are these? }\end{array}$ & & \\
\hline
\end{tabular}


Copies of this publication can be obtained from:

DfES Publications

P.O. Box 5050

Sherwood Park

Annesley

Nottingham

NG15 ODJ

Tel: 08456022260

Fax: 08456033360

Minicom: 08456055560

Oneline: www.dfespublications.gov.uk

(c) Queen's Printer and Controller of HMSO 2005.

Produced by the Department for Education and Skills

ISBN 1844784584

Ref No: RR636

www.dfes.go.uk/research 